\title{
FINITE DIMENSIONAL IRREDUCIBLE REPRESENTATIONS OF FINITE $W$-ALGEBRAS ASSOCIATED TO EVEN MULTIPLICITY NILPOTENT ORBITS IN CLASSICAL LIE ALGEBRAS
}

\author{
JONATHAN S. BROWN AND SIMON M. GOODWIN
}

\begin{abstract}
We consider finite $W$-algebras $U(\mathfrak{g}, e)$ associated to even multiplicity nilpotent elements in classical Lie algebras. We give a classification of finite dimensional irreducible $U(\mathfrak{g}, e)$-modules with integral central character in terms of the highest weight theory from [BGK]. As a corollary, we obtain a parametrization of primitive ideals of $U(\mathfrak{g})$ with associated variety the closure of the adjoint orbit of $e$ and integral central character.
\end{abstract}

\section{INTRODUCTION}

Let $\mathfrak{g}$ be a reductive Lie algebra over $\mathbb{C}$ and let $e \in \mathfrak{g}$ be nilpotent. The finite $W$ algebra $U(\mathfrak{g}, e)$ associated to the pair $(\mathfrak{g}, e)$ is a finitely generated algebra obtained from $U(\mathfrak{g})$ by a certain quantum Hamiltonian reduction. Finite $W$-algebras were introduced to the mathematical literature by Premet in [Pr1], though they appeared earlier in mathematical physics under a slightly different guise, see for example [DK]. It is proved in $\left[\mathrm{D}^{3} \mathrm{HK}\right]$ that the definition in the mathematical physics literature via BRST cohomology agrees with Premet's definition. A recent survey of the theory of finite $W$-algebras is given in [Lo4].

There is a close connection between finite dimensional irreducible representations of $U(\mathfrak{g}, e)$ and primitive ideals of $U(\mathfrak{g})$ stemming from Skryabin's equivalence, [Sk]. This link was investigated further in [Gi, Pr2, Pr3, Lo1, Lo2 culminating in [Lo2, Theorem 1.2.2], which says that there is a bijection between the primitive ideals of $U(\mathfrak{g})$ whose associated variety is the closure of the adjoint orbit of $e$, and the orbits on the component group of the centralizer of $e$ on the isomorphism classes of finite dimensional irreducible $U(\mathfrak{g}, e)$-modules. An important tool in these results is Losev's approach to $U(\mathfrak{g}, e)$ via Fedosov quantization introduced in [Lo1].

Further motivation for the study of finite $W$-algebras comes from noncommutative deformations of singularities, see [Pr1]; representation theory of modular reductive Lie algebras, see [Pr1, $\operatorname{Pr} 3,[\operatorname{Pr} 4]$; and representation theory of degenerate cyclotomic Hecke algebras, see BK3.

Despite the high level of recent interest, the representation theory of finite $W$-algebras is only well-understood in certain special cases. For $\mathfrak{g}=\mathfrak{g l}_{n}(\mathbb{C})$ a thorough study of the representation theory of $U(\mathfrak{g}, e)$ was undertaken by Brundan and Kleshchev in [BK1, BK2]. They obtained a classification of finite dimensional irreducible modules along with numerous other results. Several interesting consequences of this type A theory have been found, see [BB, Bru1, Bru2, BK3]. Recent work of the first author gives a classification of finite dimensional irreducible $U(\mathfrak{g}, e)$-modules for rectangular nilpotent orbits when $\mathfrak{g}$ is of classical type, see [Bro1, Bro2].

2010 Mathematics Subject Classification: 17B10, 81R05. 
In [BGK], a highest weight theory for representations of finite $W$-algebras was developed. Verma modules for $U(\mathfrak{g}, e)$ are defined and it is shown that any finite dimensional irreducible $U(\mathfrak{g}, e)$-module is isomorphic to the irreducible head of a Verma module. The classifications of finite dimensional irreducible $U(\mathfrak{g}, e)$-modules for the cases considered in [BK2] and [Bro2] can be described nicely in terms of this highest weight theory, see [BGK, §5.2] and [Bro2, $\S 5]$.

Let $\mathfrak{g}$ be a classical Lie algebra. We say a nilpotent element $e \in \mathfrak{g}$ is even multiplicity if all parts of the Jordan decomposition of $e$ have even multiplicity. In this paper we give a classification of finite dimensional irreducible $U(\mathfrak{g}, e)$-modules when $e$ is even multiplicity in terms of the highest weight theory from [BGK], see Theorem 5.13. Next we introduce some notation required to state Theorem 1.1. which is Theorem 5.13 in the special case that all parts of the Jordan decomposition of $e$ have the same parity.

Let $\tilde{G}=\operatorname{Sp}_{2 n}(\mathbb{C})\left(\right.$ or $\left.\mathrm{O}_{2 n}(\mathbb{C})\right)$, let $G=\tilde{G}^{\circ}$, and let $\mathfrak{g}=\operatorname{Lie} G$, so $\mathfrak{g}=\mathfrak{s p}_{2 n}=\mathfrak{s p}_{2 n}(\mathbb{C})$ (or $\mathfrak{s o}_{2 n}=\mathfrak{s o}_{2 n}(\mathbb{C})$ ). Let $V \cong \mathbb{C}^{2 n}$ denote the natural $\mathfrak{g}$-module with standard basis $e_{-n}, \ldots, e_{-1}, e_{1}, \ldots, e_{n}$, and $\mathfrak{g}$-invariant skew-symmetric (or symmetric) bilinear form $(.,$. ) defined by $\left(e_{i}, e_{j}\right)=\left(e_{-i}, e_{-j}\right)=0$ and $\left(e_{i}, e_{-j}\right)=\delta_{i, j}$ for $1 \leq i, j \leq n$. Let $\left\{e_{i, j} \mid i, j=\right.$ $\pm 1, \ldots, \pm n\}$ be the standard basis of $\mathfrak{g l}_{2 n}=\mathfrak{g l}_{2 n}(\mathbb{C}) \cong \mathfrak{g l}(V)$. Now $\mathfrak{g}$ is spanned by elements of the form $f_{i, j}=e_{i, j}+\eta_{i, j} e_{-j,-i}$ for $i, j= \pm 1, \cdots \pm n$, where $\eta_{i, j}=\operatorname{sgn}(i) \operatorname{sgn}(j)$ if $\mathfrak{g}=\mathfrak{s p}_{2 n}(\mathbb{C})$ and $\eta_{i, j}=1$ if $\mathfrak{g}=\mathfrak{s o}_{2 n}(\mathbb{C})$. Let $\mathfrak{t}=\left\langle f_{i, i} \mid i=1, \ldots, n\right\rangle$ be the standard Cartan subalgebra of $\mathfrak{g}$, and let $\Phi$ and $W$ be the root system and Weyl group of $\mathfrak{g}$ with respect to $\mathfrak{t}$. We also let $\mathfrak{b}$ be the Borel subalgebra of $\mathfrak{g}$ of upper triangular matrices in $\mathfrak{g}$, so $\mathfrak{b}$ contains $\mathfrak{t}$. Let $\Phi^{+} \subseteq \Phi$ be the corresponding set of positive roots. We write $\mathfrak{t}_{\mathbb{Z}}^{*} \subseteq \mathfrak{t}^{*}$ for the integral weight lattice.

Let $\mathbf{p}=\left(p_{1} \geq \cdots \geq p_{2 r}\right)$ be a partition of $2 n$ such that $p_{2 i-1}=p_{2 i}$ for $i=1, \ldots, r$. The symmetric pyramid associated to $\mathbf{p}$ is a diagram in the plane consisting of $2 n$ boxes of size $2 \times 2$ positioned centrally symmetric around the origin. Symmetric pyramids were defined in [EK] for all partitions corresponding to nilpotent elements in $\mathfrak{g}$, see also [BruG]. There are $p_{1}$ boxes in the middle two rows of the symmetric pyramid associated to $\mathbf{p}$, then $p_{3}$ boxes in the next two rows out, and so on. We define the coordinate pyramid coord(p) by filling the boxes of the symmetric pyramid associated to $\mathbf{p}$ with the integers $-n, \ldots,-1,1, \ldots, n$ from left to right and from top to bottom. For example, for $\mathbf{p}=(4,4,2,2)$ we have

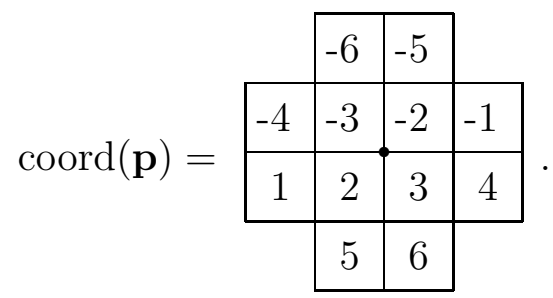

¿From $\operatorname{coord}(\mathbf{p})$ we may define the nilpotent element $e=\sum f_{i, j}$, where the sum is over positive $i$ for which the box containing $i$ is the left neighbour of the box containing $j$. Then the Jordan type of $e$ is $\mathbf{p}$, so $e$ is an even multiplicity nilpotent. In the above example, $e=f_{1,2}+f_{2,3}+f_{3,4}+f_{5,6}$.

For the remainder of the introduction we assume that all parts of $\mathbf{p}$ have the same parity, though this condition is not necessary for Theorem 5.13 .

Given $i \in\{ \pm 1, \ldots, \pm n\}$, let $\operatorname{col}(i)$ be the column of $i$, i.e the $x$-coordinate of the centre of the box labelled by $i$. We define row $(i)$ analogously (however we use a different meaning for 
row $(i)$ in Sections 4 and 51). We define

$$
\begin{gathered}
\mathfrak{p}=\left\langle f_{i, j} \mid \operatorname{col}(i) \leq \operatorname{col}(j)\right\rangle, \quad \mathfrak{m}=\left\langle f_{i, j} \mid \operatorname{col}(i)>\operatorname{col}(j)\right\rangle, \\
\mathfrak{h}=\left\langle f_{i, j} \mid \operatorname{col}(i)=\operatorname{col}(j)\right\rangle, \quad \mathfrak{g}_{0}=\left\langle f_{i, j} \mid \operatorname{row}(i)=\operatorname{row}(j)\right\rangle .
\end{gathered}
$$

Then $\mathfrak{p}$ is a parabolic subalgebra of $\mathfrak{g}$ with Levi subalgebra $\mathfrak{h}$, and $\mathfrak{m}$ is the nilradical of the opposite parabolic to $\mathfrak{p}$. Also $\mathfrak{g}_{0}$ is a minimal Levi subalgebra of $\mathfrak{g}$ containing $e$, and $e$ is a regular nilpotent element of $\mathfrak{g}_{0}$. We write $\Phi_{0}$ and $W_{0}$ for the root system and Weyl group of $\mathfrak{g}_{0}$ with respect to $\mathfrak{t}$, and let $\Phi_{0}^{+}=\Phi_{0} \cap \Phi^{+}$.

We define $\mathfrak{m}_{\chi}=\{x-\chi(x) \mid x \in \mathfrak{m}\} \subseteq U(\mathfrak{g})$, where $\chi \in \mathfrak{g}^{*}$ is dual to $e$ via the trace form, and let $Q_{\chi}$ be the $U(\mathfrak{g})$-module $U(\mathfrak{g}) / U(\mathfrak{g}) \mathfrak{m}_{\chi}$. We note that $Q_{\chi}$ is isomorphic to the induced module $U(\mathfrak{g}) \otimes_{U(\mathfrak{m})} \mathbb{C}_{\chi}$, where $\mathbb{C}_{\chi}$ is the 1-dimensional $U(\mathfrak{m})$-module given by $\chi$. The finite $W$-algebra associated to $e$ is defined to be

$$
U(\mathfrak{g}, e)=\operatorname{End}_{U(\mathfrak{g})}\left(Q_{\chi}\right)^{\text {op }} .
$$

By the PBW theorem we have $Q_{\chi} \cong U(\mathfrak{p})$ as vector spaces, then by a Frobenius reciprocity argument $U(\mathfrak{g}, e)$ is isomorphic to the subalgebra of $U(\mathfrak{p})$ of twisted $\mathfrak{m}$-invariants:

$$
U(\mathfrak{g}, e) \cong\left\{u \in U(\mathfrak{p}) \mid[x, u] \in U(\mathfrak{g}) \mathfrak{m}_{\chi} \text { for all } x \in \mathfrak{m}\right\} .
$$

By [BGK, Theorem 4.3 and Lemma 5.1], there is a certain subquotient of $U(\mathfrak{g}, e)$ isomorphic to $S(\mathfrak{t})^{W_{0}}$, see also Theorem 3.2 and $\$ 3.5$. Verma modules for $U(\mathfrak{g}, e)$ are obtained by "inducing" irreducible $S(\mathfrak{t})^{W_{0}}$-modules, see [BGK, §4.2] or $§ 3.3$. The finite dimensional irreducible $S(\mathfrak{t})^{W_{0}}$-modules are given by the set $\mathfrak{t}^{*} / W_{0}$ of $W_{0}$-orbits in $\mathfrak{t}^{*}$. Given $\Lambda \in \mathfrak{t}^{*} / W_{0}$, we write $M(\Lambda)$ for the Verma module corresponding to $\Lambda$. By [BGK, Theorem 4.5], $M(\Lambda)$ has an irreducible head, denoted $L(\Lambda)$, and any finite dimensional irreducible $U(\mathfrak{g}, e)$-module is isomorphic to $L(\Lambda)$ for some $\Lambda \in \mathfrak{t}^{*} / W_{0}$. We note that in our labelling we have built in "shifts", which we do not mention here. These shifts are given in Section 3, where we review highest weight theory.

We use $\operatorname{Pyr}(\mathbf{p})$ to denote the set of skew-symmetric fillings of the boxes of the symmetric pyramid associated to $\mathbf{p}$ by elements of $\mathbb{C}$. We identify $A \in \operatorname{Pyr}(\mathbf{p})$ with a weight $\lambda_{A} \in \mathfrak{t}^{*}$ by setting $\lambda_{A}=\sum_{i=1}^{n} a_{i} \epsilon_{i}$, where $a_{i}$ fills the box in $A$ occupied by $i$ in $\operatorname{coord}(\mathbf{p})$ and $\epsilon_{i}=f_{-i,-i}^{*}$. For example if

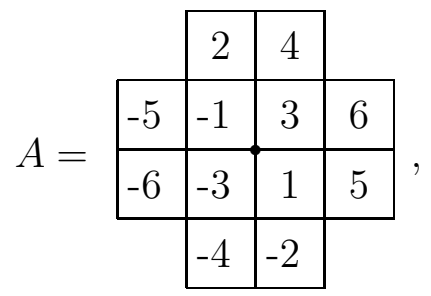

then $\lambda_{A}=2 \epsilon_{6}+4 \epsilon_{5}-5 \epsilon_{4}-\epsilon_{3}+3 \epsilon_{2}+6 \epsilon_{1}$.

The $W_{0}$-orbit $\Lambda_{A}$ of $\lambda_{A}$ identifies with the row equivalence class of $A$; we denote this row equivalence class by $\bar{A}$. We restrict attention to $\left\{A \in \operatorname{Pyr}(\mathbf{p}) \mid \lambda_{A} \in \mathfrak{t}_{\mathbb{Z}}^{*}\right\}$ in this paper, which amounts to the coefficients of $\lambda_{A}$ all lying in $\mathbb{Z}$ if $\mathfrak{g}=\mathfrak{s p}_{2 n}$, or either all lying in $\mathbb{Z}$ or all lying in $\mathbb{Z}+\frac{1}{2}$ if $\mathfrak{g}=\mathfrak{s o}_{2 n}$.

We recall that the restriction of the projection of $U(\mathfrak{g})$ onto $Q_{\chi}$ restricts to an isomorphism from the centre $Z(\mathfrak{g})$ of $U(\mathfrak{g})$ onto the centre of $U(\mathfrak{g}, e)$, see the footnote to [Pr2, Question 5.1]. This allows us to view central characters of $U(\mathfrak{g}, e)$-modules as homomorphisms $Z(\mathfrak{g}) \rightarrow \mathbb{C}$, as explained at the end of $\$ 2.2$. The Harish Chandra homomorphism gives an isomorphism 
$Z(\mathfrak{g}) \stackrel{\sim}{\rightarrow} S(\mathfrak{t})^{W}$ and this meshes well with the subquotient of $U(\mathfrak{g}, e)$ isomorphic to $S(\mathfrak{t})^{W_{0}}$ see [BGK, Theorem 4.7] or (3.7). In particular, this means that $L(\Lambda)$ has the same central character as $L\left(\Lambda^{\prime}\right)$ if and only if $\Lambda$ and $\Lambda^{\prime}$ are contained in the same $W$-orbit in $\mathfrak{t}^{*}$. In terms of the pyramids this translates to the multisets of entries of $A$ and $A^{\prime}$ being equal, where $A, A^{\prime} \in \operatorname{Pyr}(\mathbf{p})$ are such that $\Lambda_{A}=\Lambda$ and $\Lambda_{A^{\prime}}=\Lambda^{\prime}$.

The last ingredient needed for the statement of Theorem 5.13 is the component group $\tilde{C}$ of the centralizer of $e$ in $\tilde{G}$. It is well-known that $\tilde{C} \cong \tilde{H}^{e} /\left(\tilde{H}^{e}\right)^{\circ}$, where $\tilde{H}$ is the subgroup of $\tilde{G}$ corresponding to $\mathfrak{h}$ and $\tilde{H}^{e}$ is the centralizer of $e$ in $\tilde{H}$. One can see that the adjoint action of $\tilde{H}$ on $\mathfrak{g}$ induces an action of $\tilde{H}$ on $U(\mathfrak{g}, e)$. As explained in the introduction to [Lo2, this induces an action of $\tilde{C}$ on the isomorphism classes of finite dimensional irreducible $U(\mathfrak{g}, e)$-modules.

In $\$ 5.3$ we define an action of $\tilde{C}$ on a certain subset of $\operatorname{Pyr}^{\leq}(\mathbf{p})$, where $\mathrm{Pyr}^{\leq}(\mathbf{p})$ is the subset of $\operatorname{Pyr}(\mathbf{p})$ consisting of tables with weakly increasing rows. To be more specific let $\operatorname{Pyr}^{\mathrm{c}}(\mathbf{p})$ denote the subset of $\mathrm{Pyr}^{\leq}(\mathbf{p})$ consisting of elements which correspond to integral weights and are row equivalent to column strict. By column strict we mean that all of the columns are strictly decreasing. In type $\mathrm{D}$ we also call elements of $\operatorname{Pyr}(\mathbf{p})$ column strict if all of their columns are strictly decreasing, or if their columns are strictly decreasing everywhere, except the two middle boxes in the middle column (if it exists) contain 0. Now the set on which the $\tilde{C}$-action is defined is $\tilde{C} \cdot \operatorname{Pyr}^{\mathrm{c}}(\mathbf{p})$. In the future work [BroG] it will be shown that this action corresponds to the action of $\tilde{C}$ on the isomorphism classes of finite dimensional irreducible $U(\mathfrak{g}, e)$-modules.

We are now in a position to state the main theorem of this paper in the case that all parts of $\mathbf{p}$ have the same parity. The more general statement is given in Theorem 5.13 .

Theorem 1.1. Let $\mathfrak{g}=\mathfrak{s p}_{2 n}$ or $\mathfrak{s o}_{2 n}$, let $\mathbf{p}$ be as above, let $e \in \mathfrak{g}$ be an even multiplicity nilpotent such that the Jordan type of e is $\mathbf{p}$, and let $A \in \mathrm{Pyr} \leq(\mathbf{p})$ be such that $\Lambda_{A} \in \mathfrak{t}_{\mathbb{Z}}^{*} / W_{0}$. Then the irreducible $U(\mathfrak{g}, e)$-module $L\left(\Lambda_{A}\right)$ is finite dimensional if and only if there exists $c \in \tilde{C}$ such that $c \cdot A \in \operatorname{Pyr}^{\mathrm{c}}(\mathbf{p})$.

We remark here that the restriction to integral weights is necessary for the theorem to hold, see Remark 5.19. We hope to address the non-integral case in future work.

Through the correspondence of finite dimensional irreducible $U(\mathfrak{g}, e)$-modules and primitive ideals of $U(\mathfrak{g})$ with associated variety $\overline{G \cdot e}$ discussed above, we obtain the following corollary. We limit this corollary to the type $\mathrm{C}$ case, since the type $\mathrm{D}$ case is more complicated. We also obtain a more general corollary from Theorem 5.13 which includes the type D case, see Corollary 5.20, For the statement, let $\rho \in \mathfrak{t}^{*}$ be the half sum of the roots in $\Phi^{+}$. Given $\lambda \in \mathfrak{t}^{*}$ we write $L(\lambda)$ for the simple highest weight $U(\mathfrak{g})$-module with highest weight $\lambda-\rho$.

Corollary 1.2. Let $\mathfrak{g}=\mathfrak{s p}_{2 n}$, and let $\mathbf{p}$, e be as in Theorem 1.1. Then

$$
\left\{\operatorname{Ann}_{U(\mathfrak{g})} L\left(\lambda_{A}\right) \mid A \in \operatorname{Pyr}^{\mathrm{c}}(\mathbf{p})\right\}
$$

is a complete set of pairwise distinct primitive ideals of $U(\mathfrak{g})$ with integral central character and associated variety $\overline{G \cdot e}$.

We now give an outline of the contents of this paper, and point out the most significant results. In Section 2 we review some basic facts about finite $W$-algebras. In Section 3 we 
review the highest weight theory for finite $W$-algebras introduced in [BGK] and generalize it to "Levi subalgebras" of finite $W$-algebras; we note that some of the results here are also contained in [Lo3]. An important result is Proposition 3.6, which gives an inductive approach to determining finite dimensional irreducible modules for finite $W$-algebras. In [Lo3] Losev proved [BGK, Conjecture 5.2], but used a potentially different isomorphism at a key technical point. In Proposition 3.12, we show that these isomorphisms are the same, this completes the verification of [BGK, Conjecture 5.2]. Also in \$3.6, we discuss how the action

of the component group $\tilde{C}$ interacts with highest weight theory. In Section 4 we prove a variety of combinatorial results about generalizations of tableaux and pyramids called tables and s-tables. The key result is Theorem 4.6, which relates a table being row equivalent to column strict to the output of the Robinson-Schensted algorithm applied to the word of the table. In Section 5 we prove Theorem [5.13. The key ingredients are Corollary [3.13, Theorem 4.6 and the algorithm of Barbasch and Vogan for calculating the associated variety of a primitive ideal in the universal enveloping algebra of a classical Lie algebra.

Acknowledgments. This research is funded by EPSRC grant EP/G020809/1.

\section{Preliminaries}

In this section we define the finite $W$-algebra $U(\mathfrak{g}, e)$ associated to a nilpotent element $e$ in a reductive Lie algebra $\mathfrak{g}$. Then we recall some basic properties of $U(\mathfrak{g}, e)$ that we require later in the paper. The definition we give here is the definition "via non-linear Lie algebras" from [BGK, §2.2], which is essentially the same as the Whittaker model definition given in [Pr1], see [Pr2, §2.4] and [BGK, Theorem 2.4].

2.1. Notation and definition of $U(\mathfrak{g}, e)$. Let $\mathfrak{g}$ be the Lie algebra of a reductive algebraic group $\tilde{G}$ over $\mathbb{C}$, and let $G=\tilde{G}^{\circ}$. Let $(\cdot \mid \cdot)$ be a non-degenerate symmetric invariant bilinear form on $\mathfrak{g}$. For $x \in \mathfrak{g}$ and a subspace $\mathfrak{a}$ of $\mathfrak{g}$, we write $\mathfrak{a}^{x}=\{y \in \mathfrak{a} \mid[y, x]=0\}$ for the centralizer of $x$ in $\mathfrak{a}$; for a subgroup $A$ of $\tilde{G}$ we write $A^{x}$ for the centralizer of $x$ in $A$.

Let $\mathfrak{t}$ be a maximal toral subalgebra of $\mathfrak{g}$. We write $\Phi \subseteq \mathfrak{t}^{*}$ for the root system of $\mathfrak{g}$ with respect to $\mathfrak{t}$. The usual pairing between $\mathfrak{t}^{*}$ and $\mathfrak{t}$ is denoted by $\langle\cdot, \cdot\rangle$. We note that $(\cdot \mid \cdot)$ induces a nondegenerate symmetric form on both $\mathfrak{t}$ and $\mathfrak{t}^{*}$. For $\alpha \in \Phi$ we write $\alpha^{\vee}$ for the corresponding coroot.

Let $e \in \mathfrak{g}$ be a nilpotent element, and define the linear map $\chi: \mathfrak{g} \rightarrow \mathbb{C}$ by $\chi(x)=(e \mid x)$. By the Jacobson-Morozov theorem, we can find $h, f \in \mathfrak{g}$ so that $(e, h, f)$ is an $\mathfrak{s l}_{2}$-triple in g. Let

$$
\mathfrak{g}=\bigoplus_{j \in \mathbb{Z}} \mathfrak{g}(j)
$$

be the ad $h$-eigenspace decomposition, i.e. $\mathfrak{g}(j)=\{x \in \mathfrak{g} \mid[h, x]=j x\}$.

We define the following subspaces of $\mathfrak{g}$

$$
\mathfrak{p}=\bigoplus_{j \geq 0} \mathfrak{g}(j), \quad \mathfrak{n}=\bigoplus_{j<0} \mathfrak{g}(j), \quad \mathfrak{m}=\bigoplus_{j \leq-2} \mathfrak{g}(j), \quad \mathfrak{h}=\mathfrak{g}(0), \quad \mathfrak{k}=\mathfrak{g}(-1)
$$

In particular, $\mathfrak{p}$ is a parabolic subalgebra of $\mathfrak{g}$ with Levi factor $\mathfrak{h}$ and $\mathfrak{n}$ is the nilradical of the opposite parabolic. We let $b_{1}, \ldots, b_{r}$ be a homogeneous basis for $\mathfrak{n}$ such that $b_{i} \in \mathfrak{g}\left(-d_{i}\right)$ and has weight $\beta_{i} \in \Phi$, where $d_{i} \in \mathbb{Z}_{>0}$. 
To formulate the definition of $U(\mathfrak{g}, e)$ we use an easy special case of the notion of a nonlinear Lie superalgebra from [DK, Definition 3.1], where the grading is concentrated in degree zero, see [BGK, §2.2] for the definition of nonlinear Lie algebras in this case.

We define a symplectic form $\langle\cdot \mid \cdot\rangle$ on $\mathfrak{k}$ by $\langle x \mid y\rangle=\chi([y, x])$. Let $\mathfrak{k}^{\text {ne }}=\left\{x^{\text {ne }} \mid x \in \mathfrak{k}\right\}$ be a "neutral" copy of $\mathfrak{k}$. We write $x^{\text {ne }}=x(-1)^{\text {ne }}$ for any element $x \in \mathfrak{g}$. Now make $\mathfrak{k}^{\text {ne }}$ into a non-linear Lie algebra with non-linear Lie bracket defined by $\left[x^{\mathrm{ne}}, y^{\mathrm{ne}}\right]=\langle x \mid y\rangle$ for $x, y \in \mathfrak{k}$. Note that $U\left(\mathfrak{k}^{\mathrm{ne}}\right)$ is isomorphic to the Weyl algebra associated to $\mathfrak{k}$ and the form $\langle\cdot \mid \cdot\rangle$. We view $\tilde{\mathfrak{g}}=\mathfrak{g} \oplus \mathfrak{k}^{\text {ne }}$ as a non-linear Lie algebra with bracket obtained by extending the brackets already defined on $\mathfrak{g}$ and $\mathfrak{k}^{\text {ne }}$ to all of $\tilde{\mathfrak{g}}$, and declaring $\left[x, y^{\text {ne }}\right]=0$ for $x \in \mathfrak{g}, y \in \mathfrak{k}$. Then $U(\tilde{\mathfrak{g}}) \cong U(\mathfrak{g}) \otimes U\left(\mathfrak{k}^{\text {ne }}\right)$. Also let $\tilde{\mathfrak{p}}=\mathfrak{p} \oplus \mathfrak{k}^{\text {ne }}$; this is a subalgebra of $\tilde{\mathfrak{g}}$ whose universal enveloping algebra is identified with $U(\mathfrak{p}) \otimes U\left(\mathfrak{k}^{\text {ne }}\right)$.

We define $\tilde{\mathfrak{n}}_{\chi}=\left\{x-x^{\text {ne }}-\chi(x) \mid x \in \mathfrak{n}\right\}$. By the PBW theorem for $U(\tilde{\mathfrak{g}})$ we have a direct sum decomposition $U(\tilde{\mathfrak{g}})=U(\tilde{\mathfrak{p}}) \oplus U(\tilde{\mathfrak{g}}) \tilde{\mathfrak{n}}_{\chi}$. We write $\operatorname{Pr}: U(\tilde{\mathfrak{g}}) \rightarrow U(\tilde{\mathfrak{p}})$ for the projection along this direct sum decomposition. We define the finite $W$-algebra

$$
U(\mathfrak{g}, e)=U(\tilde{\mathfrak{p}})^{\mathfrak{n}}=\left\{u \in U(\tilde{\mathfrak{p}}) \mid \operatorname{Pr}\left(\left[x-x^{\mathrm{ne}}, u\right]\right)=0 \text { for all } x \in \mathfrak{n}\right\} .
$$

It is a subalgebra of $U(\tilde{\mathfrak{p}})$ by [BGK, Theorem 2.4].

Remark 2.1. We note that in case the grading $\mathfrak{g}=\bigoplus_{j \in \mathbb{Z}} \mathfrak{g}(j)$ is even, meaning that $\mathfrak{g}(j)=0$ for odd $j$, we have $\mathfrak{k}=0$. So we do not require nonlinear Lie algebras and $U(\mathfrak{g}, e) \subseteq U(\mathfrak{p})$; this is the case for the definition of $U(\mathfrak{g}, e)$ given in the introduction.

\subsection{Basic properties of $U(\mathfrak{g}, e)$. A Lie algebra homomorphism}

$$
\theta: \mathfrak{g}^{e} \hookrightarrow U(\tilde{\mathfrak{p}})
$$

is defined in [BGK, Theorem 3.3] reformulating the definition given in [Pr2, §2.5]. The restriction of $\theta$ to $\mathfrak{h}^{e}$ gives an inclusion $\mathfrak{h}^{e} \hookrightarrow U(\mathfrak{g}, e)$, allowing us to view $\mathfrak{h}^{e}$ as a subalgebra of $U(\mathfrak{g}, e)$. In particular this gives an adjoint action of $\mathfrak{h}^{e}$ on $U(\mathfrak{g}, e)$ and $U(\tilde{\mathfrak{p}})$.

We can modify $\theta$ to obtain a (non-unique) $\mathfrak{h}^{e}$-equivariant linear map

$$
\Theta: \mathfrak{g}^{e} \rightarrow U(\mathfrak{g}, e)
$$

as in [BGK, Theorem 3.6], which is essentially a restatement of [Pr1, Theorem 4.6]. Then for a basis $x_{1}, \ldots, x_{r}$ of $\mathfrak{g}^{e}$ the set of monomials

$$
\left\{\Theta\left(x_{1}\right)^{a_{1}} \ldots \Theta\left(x_{r}\right)^{a_{r}} \mid a_{1}, \ldots, a_{r} \in \mathbb{Z}_{\geq 0}\right\}
$$

forms a PBW basis of $U(\mathfrak{g}, e)$.

We let $\tilde{C}=\tilde{G}^{e} /\left(\tilde{G}^{e}\right)^{\circ}$ be the component group of the centralizer $\tilde{G}^{e}$ of $e$ in $\tilde{G}$. For connected $G$, it is a standard result that $C=G^{e} /\left(G^{e}\right)^{\circ} \cong H^{e} /\left(H^{e}\right)^{\circ}$, where $H$ is the connected subgroup of $G$ corresponding to $\mathfrak{h}$. This can be proved by noting that the centralizer of $G$ must normalize $\mathfrak{p}$, see [Ja, Proposition 5.9], and that any two Levi subalgebras of $\mathfrak{p}$ are conjugate by an element of the unipotent radical of $P$, where $P$ is the parabolic subgroup of $G$ corresponding to $\mathfrak{p}$. These arguments work just as well for $\tilde{G}$, so we have $\tilde{C} \cong \tilde{H}^{e} /\left(\tilde{H}^{e}\right)^{\circ}$ in general. It is straightforward to see that the adjoint action of $\tilde{H}^{e}$ on $\mathfrak{g}$ gives rises to an action of $\tilde{H}^{e}$ on $U(\mathfrak{g}, e)$.

Lastly we consider the centre of $U(\mathfrak{g}, e)$. The footnote to [Pr2, Question 5.1] says that the restriction of $\operatorname{Pr}$ to $Z(\mathfrak{g})$ gives an isomorphism $Z(\mathfrak{g}) \stackrel{\sim}{\rightarrow} Z(\mathfrak{g}, e)$, where $Z(\mathfrak{g}, e)$ denotes the centre of $U(\mathfrak{g}, e)$. Let $L$ be an irreducible $U(\mathfrak{g}, e)$-module. Then the centre $Z(\mathfrak{g}, e)$ of 
$U(\mathfrak{g}, e)$ acts on $L$ via a character. We say that $L$ is of central character $\psi: Z(\mathfrak{g}) \rightarrow \mathbb{C}$ if $\operatorname{Pr}(z) v=\psi(z) v$ for all $z \in Z(\mathfrak{g})$ and $v \in L$.

2.3. Losev's map of ideals. In [Lo2 Losev shows that there exists a map ${ }^{\dagger}$ from the set of ideals of $U(\mathfrak{g}, e)$ to the set of ideals of $U(\mathfrak{g})$ such that the restriction

$$
I \mapsto I^{\dagger}: \operatorname{Prim}_{0} U(\mathfrak{g}, e) \rightarrow \operatorname{Prim}_{e} U(\mathfrak{g})
$$

is a surjection. Here $\operatorname{Prim}_{0} U(\mathfrak{g}, e)$ denotes the primitive ideals of $U(\mathfrak{g}, e)$ of finite codimension, and $\operatorname{Prim}_{e} U(\mathfrak{g})$ denotes the primitive ideals of $U(\mathfrak{g})$ with associated variety equal to $\overline{G . e}$. For a definition of associated varieties, see for example [Ja, §9].

The set $\operatorname{Prim}_{0} U(\mathfrak{g}, e)$ identifies naturally with the set of isomorphism classes of finite dimensional irreducible $U(\mathfrak{g}, e)$-modules. The action of $H^{e}$ on $U(\mathfrak{g}, e)$ induces an action on $\operatorname{Prim}_{0}\left(U(\mathfrak{g}, e)\right.$. The action of $\mathfrak{h}^{e}$ of $U(\mathfrak{g}, e)$ obtained from differentiating the action of $H^{e}$ coincides with the adjoint action of $\mathfrak{h}^{e}$ through $\theta$, see for example [BGK, Theorem 3.3(i)]. Therefore, the action $H^{e}$ on $\operatorname{Prim}_{0} U(\mathfrak{g}, e)$ factors through $C$, as explained in the introduction to [Lo2. Putting this all together we obtain an action of $C$ on the set of isomorphism classes of finite dimensional $U(\mathfrak{g}, e)$-modules. We note that this action can also be described in terms of "twisting" the action of $U(\mathfrak{g}, e)$ on its finite dimensional irreducible modules.

In [Lo1, Theorem 1.2.2] and [Lo2, Theorem 1.2.2] the following properties of.$^{\dagger}$ are established:

(i) The fibers of the restriction of $\cdot^{\dagger}$ in (2.4) are precisely the $C$-orbits in $\operatorname{Prim}_{0} U(\mathfrak{g}, e)$.

(ii) Central characters are preserved by ${ }^{\dagger}$ in the sense that if $L$ is an irreducible $U(\mathfrak{g}, e)$ module with central character $\psi: Z(\mathfrak{g}) \rightarrow \mathbb{C}$, then $\left(\operatorname{Ann}_{U(\mathfrak{g}, e)} M\right)^{\dagger} \cap Z(\mathfrak{g})=\operatorname{ker} \psi$.

\section{Highest Weight theory AND "Levi subalgebras" of $U(\mathfrak{g}, e)$}

In this section we review the highest weight theory for finite $W$-algebras from BGK]. Furthermore, we extend some of the results from loc. cit. to define certain subquotients of $U(\mathfrak{g}, e)$ that play the role of Levi subalgebras; they are isomorphic to smaller finite $W$-algebras. Such subquotients were first used to study the representation theory of finite $W$-algebras by Losev in Lo3]. This isomorphism is recorded in Theorem 3.2 and is a generalization of [BGK, Theorem 4.3]. Using Theorem 3.2 we set up an inductive approach to determining the finite dimensional irreducible modules for $U(\mathfrak{g}, e)$ as set out in Proposition 3.6. A number of the results involved are straightforward generalizations from [BGK, §4], some of which are contained in [Lo3]. As our setup is slightly different to that in [Lo3], we include all statements here.

Of particular importance in this section is Corollary 3.13, which completes the verification of [BGK, Conjecture 5.2]. This gives a combinatorial criteria for an irreducible highest weight module for $U(\mathfrak{g}, e)$ to be finite dimensional in the case $e$ is of standard Levi type.

3.1. Notation for highest weight theory. It is a standard result that a Levi factor of $\mathfrak{g}^{e}$ is given by $\mathfrak{h}^{e}$. We may assume that our maximal toral subalgebra $\mathfrak{t}$ is contained in $\mathfrak{h}=\mathfrak{g}(0)$ so that $\mathfrak{t}^{e}$ is a maximal toral subalgebra of $\mathfrak{h}^{e}$. We let $\mathfrak{g}_{0}=\left\{x \in \mathfrak{g} \mid[t, x]=0\right.$ for all $\left.t \in \mathfrak{t}^{e}\right\}$ be the centralizer of $\mathfrak{t}^{e}$ in $\mathfrak{g}$. Then $\mathfrak{g}_{0}$ is a Levi subalgebra of $\mathfrak{g}$ and $e$ is a distinguished nilpotent element of $\mathfrak{g}_{0}$. We choose a Borel subalgebra $\mathfrak{b}_{0}$ of $\mathfrak{g}_{0}$ contained in $\mathfrak{p}$ and containing $\mathfrak{t}$. We write $\Phi_{0}$ for the root system of $\mathfrak{g}_{0}$ with respect to $\mathfrak{t}$ and $\Phi_{0}^{+}$for the positive roots determined by $\mathfrak{b}_{0}$. 
We choose a parabolic subalgebra $\mathfrak{q}$ of $\mathfrak{g}$ with Levi factor $\mathfrak{g}_{0}$. We write $\mathfrak{q}_{u}$ for the nilradical of $\mathfrak{q}$. Then $\mathfrak{b}=\mathfrak{b}_{0} \oplus \mathfrak{q}_{u}$ is a Borel subalgebras of $\mathfrak{g}$. The system of positive roots determined by $\mathfrak{b}$ is denoted by $\Phi^{+}$and we let $\rho=\frac{1}{2} \sum_{\alpha \in \Phi^{+}} \alpha$.

We say a subalgebra $\mathfrak{r}$ of $\mathfrak{t}^{e}$ is a full subalgebra if $\mathfrak{r}$ is equal to the centre of $\mathfrak{g}^{\mathfrak{r}}=\{x \in$ $\mathfrak{g} \mid[t, x]=0$ for all $t \in \mathfrak{r}\}$. For a full subalgebra $\mathfrak{r}$ of $\mathfrak{t}^{e}$ there is an adjoint action of $\mathfrak{r}$ on $\tilde{\mathfrak{g}}$, which extends the adjoint action of $\mathfrak{r}$ on $\mathfrak{g}$, created by declaring that $\mathfrak{r}$ acts on $\mathfrak{k}^{\text {ne }}$ by $\left[t, x^{\mathrm{ne}}\right]=[t, x]^{\text {ne }}$ for $t \in \mathfrak{r}$ and $x \in \mathfrak{k}$. For an $\mathfrak{r}$-stable subspace $\mathfrak{a}$ of $\tilde{\mathfrak{g}}$, we define $\mathfrak{a}^{\mathfrak{r}}=\{x \in \mathfrak{a} \mid[t, x]=0$ for all $t \in \mathfrak{r}\}$. We note that $\mathfrak{g}^{\mathfrak{r}}$ is a reductive Levi subalgebra of $\mathfrak{g}$, and $e, h, f \in \mathfrak{g}^{\mathfrak{r}}$, so we can define the finite $W$-algebra $U\left(\mathfrak{g}^{\mathfrak{r}}, e\right)$ as in $₫ 2.1$. We also have that $\mathfrak{g}_{0} \subseteq \mathfrak{g}^{\mathfrak{r}}$, so $\mathfrak{g}^{\mathfrak{r}}$ is an "intermediate" Levi subalgebra lying over $\mathfrak{g}_{0}$.

Now let $\mathfrak{r}$ and $\mathfrak{s}$ be full subalgebras of $\mathfrak{t}^{e}$ with $\mathfrak{r} \subseteq \mathfrak{s}$. Then we can form the $\mathfrak{s}$-weight space decomposition

$$
\mathfrak{g}^{\mathfrak{r}}=\mathfrak{g}^{\mathfrak{s}} \oplus \bigoplus_{\alpha \in \Phi_{\mathfrak{s}}^{\mathfrak{r}}} \mathfrak{g}_{\alpha}^{\mathfrak{r}}
$$

of $\mathfrak{g}^{\mathfrak{r}}$, where $\Phi_{\mathfrak{s}}^{\mathfrak{r}} \subseteq \mathfrak{s}^{*}$ and $\mathfrak{g}_{\alpha}^{\mathfrak{r}}=\left\{x \in \mathfrak{g}^{\mathfrak{r}} \mid[s, x]=\alpha(s) x\right.$ for all $\left.s \in \mathfrak{s}\right\}$. Then $\Phi_{\mathfrak{s}}^{\mathfrak{r}}$ is a restricted root system, see [BruG, $\S 2$ and $\S 3]$ for information on restricted root systems. More generally, for any subspace $\mathfrak{a}$ of $\tilde{\mathfrak{g}}^{\mathfrak{r}}$ stable under the adjoint action of $\mathfrak{s}$ we have an $\mathfrak{s - w e i g h t ~ s p a c e ~ d e c o m p o s i t i o n s ~}$

$$
\mathfrak{a}=\mathfrak{a}^{\mathfrak{s}} \oplus \bigoplus_{\alpha \in \Phi_{\mathfrak{s}}^{\mathfrak{r}}} \mathfrak{a}_{\alpha}
$$

We let $\mathfrak{q}_{\mathfrak{s}}^{\mathfrak{r}}$ be the parabolic subalgebra of $\mathfrak{g}^{\mathfrak{r}}$ with Levi factor $\mathfrak{g}^{\mathfrak{s}}$ and which contains the parabolic subalgebra $\mathfrak{q}^{\mathfrak{r}}$ of $\mathfrak{g}^{\mathfrak{r}}$. As explained in [BruG, §2], the parabolic subalgebra $\mathfrak{q}_{\mathfrak{s}}^{\mathfrak{r}}$ gives a system $\Phi_{\mathfrak{s},+}^{\mathfrak{r}}$ of positive restricted roots of $\Phi_{\mathfrak{s}}^{\mathfrak{r}}$, namely, $\Phi_{\mathfrak{s},+}^{\mathfrak{r}}=\left\{\alpha \in \Phi_{\mathfrak{s}}^{\mathfrak{r}} \mid \mathfrak{g}_{\alpha}^{\mathfrak{r}} \subseteq \mathfrak{q}_{\mathfrak{s}}^{\mathfrak{r}}\right\}$. We set $\Phi_{\mathfrak{s},-}^{\mathfrak{r}}=-\Phi_{\mathfrak{s},+}^{\mathfrak{r}}$.

In much of the notation introduced above and in the next section, there are superscripts $\mathfrak{r}$ and subscripts $\mathfrak{s}$. In the case $\mathfrak{r}=0$, we omit this superscript, so for example we write $\mathfrak{q}_{\mathfrak{s}}$ instead of $\mathfrak{q}_{\mathfrak{s}}^{0}$, and $\Phi_{\mathfrak{s}}$ rather than $\Phi_{\mathfrak{s}}^{0}$. For the case $\mathfrak{s}=\mathfrak{t}^{e}$, we omit the subscript $\mathfrak{s}$, so for example we write $\mathfrak{q}^{\mathfrak{r}}$ instead of $\mathfrak{q}_{\mathfrak{t}}^{\mathfrak{r}}$. We break this convention for the restricted root systems and write $\left(\Phi^{\mathfrak{r}}\right)^{e}$ rather than $\Phi_{\mathfrak{t}^{e}}^{\mathfrak{r}}$. Finally, in case $\mathfrak{r}=\mathfrak{t}^{e}$ we replace superscript $\mathfrak{r}$ with subscript 0 , as in $\mathfrak{g}_{0}=\mathfrak{g}^{\mathfrak{e}^{e}}$.

We give a piece of notation that is used frequently in the remainder of this paper. Given a character $\gamma: \mathfrak{a} \rightarrow \mathbb{C}$ of a Lie algebra $\mathfrak{a}$, we define, the shift automorphism $S_{\gamma}: U(\mathfrak{a}) \rightarrow U(\mathfrak{a})$ by

$$
S_{\gamma}(x)=x+\gamma(x)
$$

for each $x \in \mathfrak{a}$.

3.2. "Levi subalgebras". Let $\mathfrak{r}$ and $\mathfrak{s}$ be full subalgebras of $\mathfrak{t}^{e}$, with $\mathfrak{r} \subseteq \mathfrak{s}$.

The analogue $\theta^{\mathfrak{r}}$ of $\theta$ from (2.2) gives an adjoint action of $\left(\mathfrak{h}^{\mathfrak{r}}\right)^{e}$ on $U\left(\mathfrak{g}^{\mathfrak{r}}, e\right)$, which restricts to $\mathfrak{s}$. Therefore, we have weight space decompositions

$$
U\left(\tilde{\mathfrak{p}}^{\mathfrak{r}}\right)=U\left(\tilde{\mathfrak{p}}^{\mathfrak{r}}\right)^{\mathfrak{s}} \oplus \bigoplus_{\alpha \in \mathbb{Z} \Phi_{\mathfrak{s}}^{\mathfrak{r}} \backslash\{0\}} U\left(\tilde{\mathfrak{p}}^{\mathfrak{r}}\right)_{\alpha}
$$

and

$$
U\left(\mathfrak{g}^{\mathfrak{r}}, e\right)=U\left(\mathfrak{g}^{\mathfrak{r}}, e\right)^{\mathfrak{s}} \oplus \bigoplus_{8} U\left(\mathfrak{g}^{\mathfrak{r}}, e\right)_{\alpha}
$$


The zero weight space $U\left(\tilde{\mathfrak{p}}^{\mathfrak{r}}\right)^{\mathfrak{s}}$ is a subalgebra of $U\left(\tilde{\mathfrak{p}}^{\mathfrak{r}}\right)$. Define $U\left(\tilde{\mathfrak{p}}^{\mathfrak{r}}\right)_{\mathfrak{s}, \sharp}$ to be the left ideal of $U\left(\tilde{\mathfrak{p}}^{\mathfrak{r}}\right)$ generated by the root spaces $\tilde{\mathfrak{p}}_{\alpha}^{\mathfrak{r}}$ for $\alpha \in \Phi_{\mathfrak{s},+}^{\mathfrak{r}}$. Then, as explained in [BGK, $\S 4.1$ ], $U\left(\tilde{\mathfrak{p}}^{\mathfrak{r}}\right)_{\sharp}^{\mathfrak{s}}=U\left(\tilde{\mathfrak{p}}^{\mathfrak{r}}\right)^{\mathfrak{s}} \cap U\left(\tilde{\mathfrak{p}}^{\mathfrak{r}}\right)_{\mathfrak{s}, \sharp}$ is a two-sided ideal of $U\left(\tilde{\mathfrak{p}}^{\mathfrak{r}}\right)^{\mathfrak{s}}$ so $U\left(\tilde{\mathfrak{p}}^{\mathfrak{r}}\right)^{\mathfrak{s}}=U\left(\tilde{\mathfrak{p}}^{\mathfrak{s}}\right) \oplus U\left(\tilde{\mathfrak{p}}^{\mathfrak{r}}\right)_{\sharp}^{\mathfrak{s}}$. The projection $\pi_{\mathfrak{s}}^{\mathfrak{r}}: U\left(\tilde{\mathfrak{p}}^{\mathfrak{r}}\right)^{\mathfrak{s}} \rightarrow U\left(\tilde{\mathfrak{p}}^{\mathfrak{s}}\right)$ along this direct sum decomposition induces an isomorphism $U\left(\tilde{\mathfrak{p}}^{\mathfrak{r}}\right)^{\mathfrak{s}} / U\left(\tilde{\mathfrak{p}}^{\mathfrak{r}}\right)_{\sharp}^{\mathfrak{s}} \cong U\left(\tilde{\mathfrak{p}}^{\mathfrak{s}}\right)$.

Similarly, $U\left(\mathfrak{g}^{\mathfrak{r}}, e\right)^{\mathfrak{s}}$ is a subalgebra of $U\left(\mathfrak{g}^{\mathfrak{r}}, e\right)$ and we define $U\left(\mathfrak{g}^{\mathfrak{r}}, e\right)_{\mathfrak{s}, \sharp}$ to be the left ideal of $U\left(\mathfrak{g}^{\mathfrak{r}}, e\right)$ generated by the elements $\Theta^{\mathfrak{r}}(x)$ for $x \in\left(\mathfrak{g}^{\mathfrak{r}}\right)_{\alpha}^{e}$ and $\alpha \in \Phi_{\mathfrak{s},+}^{\mathfrak{r}}$, where $\Theta^{\mathfrak{r}}$ is the analogue of the map $\Theta$ given in (2.3). Then $U\left(\mathfrak{g}^{\mathfrak{r}}, e\right)_{\sharp}^{\mathfrak{s}}=U\left(\mathfrak{g}^{\mathfrak{r}}, e\right)^{\mathfrak{s}} \cap U\left(\mathfrak{g}^{\mathfrak{r}}, e\right)_{\mathfrak{s}, \sharp}$ is a two sided ideal of $U\left(\mathfrak{g}^{\mathfrak{r}}, e\right)^{\mathfrak{s}}$ so we can form the quotient $U\left(\mathfrak{g}^{\mathfrak{r}}, e\right)^{\mathfrak{s}} / U\left(\mathfrak{g}^{\mathfrak{r}}, e\right)_{\sharp}^{\mathfrak{s}}$. Theorem 3.3 below says that this quotient is isomorphic to $U\left(\mathfrak{g}^{\mathfrak{s}}, e\right)$.

We recall that $b_{1}, \ldots, b_{r}$ is basis of $\mathfrak{n}$ such that $b_{i} \in \mathfrak{g}\left(-d_{i}\right)$ and has weight $\beta_{i} \in \Phi$. We let $I^{\mathfrak{r}}=\left\{i\left|\beta_{i}\right|_{\mathfrak{r}}=0\right\}$ and define

$$
\gamma_{\mathfrak{s}}^{\mathfrak{r}}=\sum_{\substack{\left.i \in I^{\mathfrak{r}} \\ \beta_{i}\right|_{\mathfrak{s}} \in \Phi_{\mathfrak{s},-}^{\mathfrak{r}}}} \beta_{i} \in \mathfrak{t}^{*} .
$$

The analogue of [BGK, Lemma 4.1] says that $\gamma_{\mathfrak{s}}^{\mathfrak{r}}$ extends uniquely to a character of $\mathfrak{p}^{\mathfrak{s}}$. Therefore, we can define the shift $S_{-\gamma_{\mathfrak{s}}^{\mathfrak{r}}}: U\left(\tilde{\mathfrak{p}}^{\mathfrak{s}}\right) \rightarrow U\left(\tilde{\mathfrak{p}}^{\mathfrak{s}}\right)$ by the formula given in (3.1) for $x \in \mathfrak{p}^{\mathfrak{s}}$, and $S_{-\gamma_{\mathfrak{s}}^{\mathfrak{r}}}\left(y^{\text {ne }}\right)=y^{\text {ne }}$ for $y \in \mathfrak{k}^{\mathfrak{s}}$. The following theorem can be proved using the same arguments as for [BGK, Theorem 4.3], which deals with the case $\mathfrak{r}=0$ and $\mathfrak{s}=\mathfrak{t}^{e}$.

Theorem 3.2. The restriction of $S_{-\gamma_{\mathfrak{s}}^{\mathfrak{r}}} \circ \pi_{\mathfrak{s}}^{\mathfrak{r}}: U\left(\tilde{\mathfrak{p}}^{\mathfrak{r}}\right)^{\mathfrak{s}} \rightarrow U\left(\tilde{\mathfrak{p}}^{\mathfrak{s}}\right)$ defines a surjective algebra homomorphism $U\left(\mathfrak{g}^{\mathfrak{r}}, e\right)^{\mathfrak{s}} \rightarrow U\left(\mathfrak{g}^{\mathfrak{s}}, e\right)$ with kernel $U\left(\mathfrak{g}^{\mathfrak{r}}, e\right)_{\sharp}^{\mathfrak{s}}$. Therefore, it induces an isomorphism

$$
\bar{\pi}_{\mathfrak{s}}^{\mathfrak{r}}: U\left(\mathfrak{g}^{\mathfrak{r}}, e\right)^{\mathfrak{s}} / U\left(\mathfrak{g}^{\mathfrak{r}}, e\right)_{\sharp}^{\mathfrak{s}} \stackrel{\sim}{\rightarrow} U\left(\mathfrak{g}^{\mathfrak{s}}, e\right) .
$$

We note that Losev established a similar isomorphism in this setting in [Lo3].

3.3. Highest weight theory and "Levi subalgebras". In this subsection we recall some definitions and results about highest weight theory for $U(\mathfrak{g}, e)$ from [BGK, §4]. In fact, we work in the general setting from the previous section with $\mathfrak{r}$ and $\mathfrak{s}$ full subalgebras of $\mathfrak{t}^{e}$, whereas the case $\mathfrak{r}=0$ and $\mathfrak{s}=\mathfrak{t}^{e}$ is considered in [BGK]. All the results that we state below can be proved in exactly the same way as in [BGK], so we simply refer to results there even though we strictly mean their analogues.

Let $V$ be a $U\left(\mathfrak{g}^{\mathfrak{s}}, e\right)$-module. Then, as in [BGK, $\left.\S 4.2\right]$, we define

$$
M_{\mathfrak{s}}^{\mathfrak{r}}(V)=M_{\mathfrak{s}}^{\mathfrak{r}}\left(V, \mathfrak{q}^{\mathfrak{r}}\right)=\left(U\left(\mathfrak{g}^{\mathfrak{r}}, e\right) / U\left(\mathfrak{g}^{\mathfrak{r}}, e\right)_{\mathfrak{s}, \sharp}\right) \otimes_{U\left(\mathfrak{g}^{\mathfrak{s}}, e\right)} V
$$

where $U\left(\mathfrak{g}^{\mathfrak{r}}, e\right) / U\left(\mathfrak{g}^{\mathfrak{r}}, e\right)_{\mathfrak{s}, \sharp}$ is viewed as a right $U\left(\mathfrak{g}^{\mathfrak{s}}, e\right)$-module via the isomorphism $\bar{\pi}_{\mathfrak{s}}^{\mathfrak{r}}$ from (3.3). The formula in (3.4) defines a functor $M_{\mathfrak{s}}^{\mathfrak{r}}: U\left(\mathfrak{g}^{\mathfrak{s}}, e\right)$-mod $\rightarrow U\left(\mathfrak{g}^{\mathfrak{r}}, e\right)$-mod, which can be viewed as an analogue of parabolic induction for modules for reductive Lie algebras.

There is also a right adjoint functor to $M_{\mathfrak{s}}^{\mathfrak{r}}$, which can be viewed as an analogue of parabolic restriction. This functor is denoted by $R_{\mathfrak{s}}^{\mathfrak{r}}: U\left(\mathfrak{g}^{\mathfrak{r}}, e\right)-\bmod \rightarrow U\left(\mathfrak{g}^{\mathfrak{s}}, e\right)-\bmod$ and defined by

$$
R_{\mathfrak{s}}^{\mathfrak{r}}(V)=\left\{v \in V \mid u v=0 \text { for all } u \in U\left(\mathfrak{g}^{\mathfrak{r}}, e\right)_{\mathfrak{s}, \sharp}\right\},
$$

where the action of $U\left(\mathfrak{g}^{\mathfrak{s}}, e\right)$ is through the isomorphism in (3.3). We remark that for $v \in V \in$ $U\left(\mathfrak{g}^{\mathfrak{r}}, e\right)$-mod, we have $v \in R_{\mathfrak{s}}^{\mathfrak{r}}(V)$ if and only if $\Theta^{\mathfrak{r}}(x) v=0$ for all $x \in\left(\mathfrak{g}^{\mathfrak{r}}\right)_{\alpha}^{e}$ with $\alpha \in \Phi_{\mathfrak{s},+}^{\mathfrak{r}}$. We also note that functors similar to $M_{\mathfrak{s}}^{\mathfrak{r}}$ and $R_{\mathfrak{s}}^{\mathfrak{r}}$ were used by Losev in [Lo3]. 
Let $\left\{V_{\Lambda} \mid \Lambda \in \mathcal{L}_{\mathfrak{s}}\right\}$ be a parametrization of a complete set of pairwise non-isomorphic finite dimensional irreducible $U\left(\mathfrak{g}^{\mathfrak{s}}, e\right)$-modules. Then for $\Lambda \in \mathcal{L}_{\mathfrak{s}}$, we define the parabolic Verma module $M_{\mathfrak{s}}^{\mathfrak{r}}(\Lambda)=M_{\mathfrak{s}}^{\mathfrak{r}}\left(V_{\Lambda}\right)$. By [BGK, Theorem 4.5], $M_{\mathfrak{s}}^{\mathfrak{r}}(\Lambda)$ has an irreducible head denoted $L_{\mathfrak{s}}^{\mathfrak{r}}(\Lambda)=L_{\mathfrak{s}}^{\mathfrak{r}}(\Lambda, \mathfrak{q})$, and any finite dimensional irreducible $U\left(\mathfrak{g}^{\mathfrak{r}}, e\right)$-module is isomorphic to $L_{\mathfrak{s}}^{\mathfrak{r}}(\Lambda)$ for some $\Lambda \in \mathcal{L}_{\mathfrak{s}}$. Moreover, for $\Lambda, \Lambda^{\prime} \in \mathcal{L}_{\mathfrak{s}}$, we have that $L_{\mathfrak{s}}^{\mathfrak{r}}(\Lambda) \cong L_{\mathfrak{s}}^{\mathfrak{r}}\left(\Lambda^{\prime}\right)$ if and only if $\Lambda=\Lambda^{\prime}$. Therefore, the $L_{\mathfrak{s}}^{\mathfrak{r}}(\Lambda)$ 's parameterized by the set

$$
\mathcal{L}_{\mathfrak{s}}^{\mathfrak{r},+}=\left\{\Lambda \in \mathcal{L}_{\mathfrak{s}} \mid L_{\mathfrak{s}}^{\mathfrak{r}}(\Lambda) \text { is finite dimensional }\right\}
$$

give a complete set of pairwise non-isomorphic finite dimensional irreducible modules for $U\left(\mathfrak{g}^{\mathfrak{r}}, e\right)$.

We define

$$
\delta_{\mathfrak{s}}^{\mathfrak{r}}=\sum_{\substack{\left.i \in I^{\mathfrak{r}} \\ \beta_{i}\right|_{\mathfrak{s}} \in \Phi_{\mathfrak{s},-} \\ d_{i} \geq 2}} \beta_{i}+\frac{1}{2} \sum_{\substack{\left.i \in I^{\mathfrak{r}} \\ \beta_{i}\right|_{\mathfrak{s}} \in \Phi_{\mathfrak{s},-} \\ d_{i}=1}} \beta_{i} \in \mathfrak{t}^{*}
$$

and $\delta^{\mathfrak{r}}=\delta_{\mathfrak{t}^{e}}^{\mathfrak{r}}$. We incorporate a shift by $\delta^{\mathfrak{r}}$ into the labelling of the weight spaces, so for a $U\left(\mathfrak{g}^{\mathfrak{r}}, e\right)$-module $M$ and $\lambda \in \mathfrak{s}^{*}$, we define the $\lambda$-weight space $M_{\lambda}=\left\{m \in M \mid \theta^{\mathfrak{r}}(s) m=\right.$ $\left(\lambda(s)-\delta^{\mathfrak{r}}(s)\right) m$ for all $\left.s \in \mathfrak{s}\right\}$. The justification for this shift is given in the next paragraph, and is based on the following commutative diagram, which is a consequence of [BGK, Lemma 4.2]:

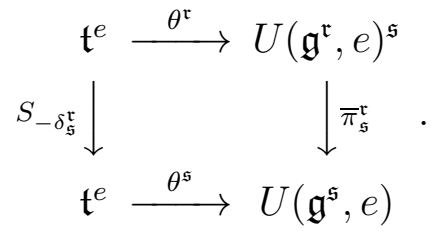

The system of positive roots $\Phi_{\mathfrak{s},+}$ allows us to define a dominance ordering on $\mathfrak{s}^{*}$ in the usual way: for $\lambda, \mu \in \mathfrak{s}^{*}$ we say $\lambda \leq \mu$ if and only if $\mu-\lambda \in \mathbb{Z}_{\geq 0} \Phi_{\mathfrak{s},+}$. Let $M$ be a $U\left(\mathfrak{g}^{\mathfrak{r}}, e\right)$ module. For $\lambda \in \mathfrak{s}^{*}$, we say that $M_{\lambda}$ is a maximal $\mathfrak{s}$-weight space of $M$ if $M_{\mu}=0$ whenever $\mu \in \mathfrak{s}^{*}$ with $\mu>\lambda$. In this case $M_{\lambda} \subseteq R_{\mathfrak{s}}^{\mathfrak{r}}(M)$, so we obtain an action of $U\left(\mathfrak{g}^{\mathfrak{s}}, e\right)$ on $M_{\lambda}$. The shift by $\delta^{\mathfrak{r}}$ in the labelling of the $\mathfrak{s}$-weight spaces means that $\theta^{\mathfrak{s}}(s) v=\left(\lambda(s)-\delta^{\mathfrak{s}}(s)\right) v$ for all $v \in M_{\lambda}$, when $M_{\lambda}$ is viewed as a $U\left(\mathfrak{g}^{\mathfrak{s}}, e\right)$-module.

We say that a $U\left(\mathfrak{g}^{\mathfrak{r}}, e\right)$-module $M$ is a highest $\mathfrak{s}$-weight module if it is generated by a maximal $\mathfrak{s}$-weight space $M_{\lambda}$ such that $M_{\lambda}$ is finite dimensional and irreducible as a $U\left(\mathfrak{g}^{\mathfrak{s}}, e\right)$ module; we say that $M$ is of type $\Lambda \in \mathcal{L}_{\mathfrak{s}}$ if $M_{\lambda}$ isomorphic to $V_{\Lambda}$. Let $M$ be a highest $\mathfrak{s}$-weight $U(\mathfrak{g}, e)$-module of type $\Lambda$. Then it follows from [BGK, Theorem 4.5] that there are unique (up to scalar) homomorphisms $M_{\mathfrak{s}}^{\mathfrak{r}}(V) \rightarrow M$ and $M \rightarrow L_{\mathfrak{s}}^{\mathfrak{r}}(V)$.

As in [BGK, $\S 4.4]$, we define $\mathcal{O}_{\mathfrak{s}}^{\mathfrak{r}}\left(e ; \mathfrak{q}_{\mathfrak{s}}^{\mathfrak{r}}\right)$ to be the category of all (finitely generated) $U\left(\mathfrak{g}^{\mathfrak{r}}, e\right)$ modules $M$ such that:

(i) the action of $\mathfrak{s}$ on $M$ is semisimple with finite dimensional $\mathfrak{s}$-weight spaces; and

(ii) the set $\left\{\lambda \in \mathfrak{s}^{*} \mid M_{\lambda} \neq 0\right\}$ is contained in a finite union of sets of the form $\left\{\nu \in \mathfrak{s}^{*} \mid\right.$ $\nu \leq \mu\}$ for $\mu \in \mathfrak{s}^{*}$.

This is an analogue of a parabolic category $\mathcal{O}$ for a reductive Lie algebra. It is easy to see that the parabolic Verma modules $M_{\mathfrak{s}}^{\mathfrak{r}}(\Lambda)$ and their irreducible heads $L_{\mathfrak{s}}^{\mathfrak{r}}(\Lambda)$ all lie in $\mathcal{O}_{\mathfrak{s}}^{\mathfrak{r}}\left(e ; \mathfrak{q}_{\mathfrak{s}}^{\mathfrak{r}}\right)$

We finish this subsection by giving, in Proposition 3.6. an inductive approach to determining finite dimensional irreducible representations of $U(\mathfrak{g}, e)$. For this proposition we require 
a transitivity property of the parabolic induction functors $M_{\mathfrak{s}}^{\mathfrak{r}}$ given in the following lemma. Recall for the statement that our notational convention is to omit superscript $\mathfrak{r}$ for $\mathfrak{r}=0$ and omit subscript $\mathfrak{s}$ for $\mathfrak{s}=\mathfrak{t}^{e}$.

Lemma 3.5. The natural multiplication map

$$
\left(U(\mathfrak{g}, e) / U(\mathfrak{g}, e)_{\mathfrak{s}, \sharp}\right) \otimes_{U\left(\mathfrak{g}^{\mathfrak{s}}, e\right)}\left(U\left(\mathfrak{g}^{\mathfrak{s}}, e\right) / U\left(\mathfrak{g}^{\mathfrak{s}}, e\right)_{\sharp}\right) \rightarrow U(\mathfrak{g}, e) / U(\mathfrak{g}, e)_{\sharp}
$$

gives rise to an isomorphism of functors

$$
M_{\mathfrak{s}} \circ M^{\mathfrak{s}} \cong M
$$

Proof. The argument required is straightforward, so we omit the details. The key point is that $\gamma=\gamma_{\mathfrak{s}}+\gamma^{\mathfrak{s}}$, which means that $\bar{\pi}=\bar{\pi}^{\mathfrak{s}} \circ \bar{\pi}_{\mathfrak{s}}$.

By our labelling convention, $\mathcal{L}$ denotes a parametrization of the set of isomorphism classes of finite dimensional irreducible $U\left(\mathfrak{g}_{0}, e\right)$-modules. The following proposition says that $\mathcal{L}^{+} \subseteq$ $\mathcal{L}^{\mathfrak{s},+}$.

Proposition 3.6. Let $\Lambda \in \mathcal{L}$, and suppose that $L(\Lambda)$ is finite dimensional. Then $L^{\mathfrak{s}}(\Lambda)$ is finite dimensional and

$$
L_{\mathfrak{s}}\left(L^{\mathfrak{s}}(\Lambda)\right) \cong L(\Lambda) .
$$

Proof. Let $\Lambda \in \mathcal{L}^{+}$, so $L=L(\Lambda)$ is finite dimensional. There exists $\lambda \in\left(\mathfrak{t}^{e}\right)^{*}$ such that $V_{\Lambda}=\left(V_{\Lambda}\right)_{\lambda}$. We consider the $U\left(\mathfrak{g}^{\mathfrak{s}}, e\right)$-module $N=R_{\mathfrak{s}}(L(\Lambda))$. The shifts in the labelling of $\mathfrak{t}^{e}$-weight spaces means that we have $N_{\lambda}$ is a maximal $\mathfrak{t}^{e}$-weight space of $N$. Also it is clear that $N_{\lambda} \cong V_{\Lambda}$ as $U\left(\mathfrak{g}_{0}, e\right)$-modules. Therefore, there is an epimorphism from the submodule of $N$ generated by $N_{\lambda}$ onto $L^{\mathfrak{s}}(\Lambda)$. Hence, $L^{\mathfrak{s}}(\Lambda)$ is finite dimensional.

We see that $L_{\mathfrak{s}}\left(L^{\mathfrak{s}}(\Lambda)\right)$ has a maximal $\mathfrak{t}^{e}$-weight space isomorphic to $V_{\Lambda}$. Therefore, there is a epimorphism $L(\Lambda) \rightarrow L_{\mathfrak{s}}\left(L^{\mathfrak{s}}(\Lambda)\right)$, which must be an isomorphism as both modules are irreducible.

3.4. Centre and central characters. Let $\mathfrak{r}$ and $\mathfrak{s}$ be a full subalgebras of $\mathfrak{t}^{e}$ with $\mathfrak{r} \subseteq \mathfrak{s}$. Recall that $\operatorname{Pr}: U(\tilde{\mathfrak{g}}) \rightarrow U(\tilde{\mathfrak{p}})$ restricts to an isomorphism $Z(\mathfrak{g}) \stackrel{\sim}{\rightarrow} Z(\mathfrak{g}, e)$. Thus the analogues $\operatorname{Pr}^{\mathfrak{r}}$ and $\operatorname{Pr}^{\mathfrak{s}}$ also restrict to isomorphisms $\operatorname{Pr}^{\mathfrak{r}}: Z\left(\mathfrak{g}^{\mathfrak{r}}\right) \stackrel{\sim}{\rightarrow} Z\left(\mathfrak{g}^{\mathfrak{r}}, e\right)$ and $\operatorname{Pr}^{\mathfrak{s}}: Z\left(\mathfrak{g}^{\mathfrak{s}}\right) \stackrel{\sim}{\rightarrow}$ $Z\left(\mathfrak{g}^{\mathfrak{s}}, e\right)$. To compare these isomorphisms we consider certain Harish-Chandra isomorphisms, which we require some notation to define. We write $W^{\mathfrak{r}}$ and $W^{\mathfrak{s}}$ for the Weyl groups with respect to $\mathfrak{t}$ of $\mathfrak{g}^{\mathfrak{r}}$ and $\mathfrak{g}^{\mathfrak{s}}$ respectively, and we write $\mathfrak{b}_{u}$ for the nilradical of $\mathfrak{b}$. Then we define $\Psi^{\mathfrak{r}}: Z\left(\mathfrak{g}^{\mathfrak{r}}\right) \stackrel{\sim}{\rightarrow} S(\mathfrak{t})^{W^{\mathfrak{r}}}$ by

$$
z \equiv S_{\rho}\left(\Psi^{\mathfrak{r}}(z)\right) \bmod U\left(\mathfrak{g}^{\mathfrak{r}}\right) \mathfrak{b}_{u}^{\mathfrak{r}}
$$

and $\Psi^{\mathfrak{s}}: Z\left(\mathfrak{g}^{\mathfrak{s}}\right) \stackrel{\sim}{\rightarrow} S(\mathfrak{t})^{W^{\mathfrak{s}}}$ is defined similarly. We recall that $\rho$ is the half sum of roots in $\Phi^{+}$, so that $\Psi^{\mathfrak{r}}$ is not the usual Harish-Chandra isomorphism for $\mathfrak{g}^{\mathfrak{r}}$, as the shift is by $\rho$ rather than a half sum of positive roots for $\mathfrak{g}^{\mathfrak{r}}$. We write $\iota_{\mathfrak{s}}^{\mathfrak{r}}: S(\mathfrak{t})^{W^{\mathfrak{r}}} \hookrightarrow S(\mathfrak{t})^{W^{\mathfrak{s}}}$ for the natural inclusion. Then the analogue of [BGK, Theorem 4.7] says that there is a unique embedding $c_{\mathfrak{s}}^{\mathfrak{r}}: Z\left(\mathfrak{g}^{\mathfrak{r}}\right) \hookrightarrow Z\left(\mathfrak{g}^{\mathfrak{s}}\right)$ such that the following diagram commutes:

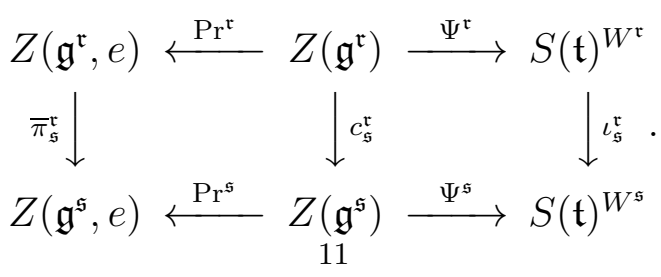


Central characters for $U\left(\mathfrak{g}^{\mathfrak{r}}, e\right)$-modules and $U\left(\mathfrak{g}^{\mathfrak{s}}, e\right)$-modules are defined as in $\$ 2.2$. Given an irreducible finite dimensional $U\left(\mathfrak{g}^{\mathfrak{s}}, e\right)$-module $V$, Schur's lemma tells us that $Z\left(\mathfrak{g}^{\mathfrak{s}}, e\right)$ acts diagonally on $V$. We write $\psi_{V}^{\mathfrak{s}}: Z\left(\mathfrak{g}^{\mathfrak{s}}\right) \rightarrow \mathbb{C}$ for the corresponding central character. Then, by [BGK, Corollary 4.8], the central character of $L_{\mathfrak{s}}^{\mathfrak{r}}(V)$ is

$$
\psi_{V}^{\mathfrak{r}}=\psi_{V}^{\mathfrak{s}} \circ c_{\mathfrak{s}}^{\mathfrak{r}}: Z\left(\mathfrak{g}^{\mathfrak{r}}\right) \rightarrow \mathbb{C} .
$$

Central characters give rise to a partition

$$
\mathcal{L}_{\mathfrak{s}}^{\mathfrak{r},+}=\bigcup_{\psi: Z\left(\mathfrak{g}^{\mathfrak{r}}\right) \rightarrow \mathbb{C}} \mathcal{L}_{\mathfrak{s}, \psi}^{\mathfrak{r},+},
$$

where $\mathcal{L}_{\mathfrak{s}, \psi}^{\mathfrak{r},+}=\left\{\Lambda \in \mathcal{L}_{\mathfrak{s}}^{\mathfrak{r},+} \mid L_{\mathfrak{s}}^{\mathfrak{r}}(\Lambda)\right.$ has central character $\left.\psi\right\}$. The following refinement of Proposition 3.6 is immediate from (3.8).

Lemma 3.10. Let $\psi: Z(\mathfrak{g}) \rightarrow \mathbb{C}$ be a character. Then

$$
\mathcal{L}_{\psi}^{+} \subseteq \bigcup_{\substack{\psi^{\prime}: Z\left(\mathfrak{g}^{\mathfrak{s}}\right) \rightarrow \mathbb{C} \\ \psi^{\prime} \circ \mathfrak{c}_{\mathfrak{s}}=\psi}} \mathcal{L}_{\psi^{\prime}}^{\mathfrak{s},+}
$$

3.5. Finite dimensional irreducible modules for standard Levi type. In this subsection, rather than working with full subalgebras $\mathfrak{r}$ and $\mathfrak{s}$ of $\mathfrak{t}^{e}$ as above, we work just in the case $\mathfrak{r}=0$ and $\mathfrak{s}=\mathfrak{t}^{e}$. We recall that we write subscript 0 instead of superscript $\mathfrak{t}^{e}$, for example $U(\mathfrak{g}, e)_{0}$ rather than $U(\mathfrak{g}, e)^{\mathfrak{t}^{e}}$.

We consider the special case where $e$ is of standard Levi type, i.e. $e$ is regular in $\mathfrak{g}_{0}$. Then $\mathfrak{k}_{0}=0$ and $\mathfrak{p}_{0}=\mathfrak{b}_{0}$ is a Borel subalgebra of $\mathfrak{g}_{0}$, and $\mathfrak{b}=\mathfrak{b}_{0} \oplus \mathfrak{q}_{u}$, where $\mathfrak{q}_{u}$ is the nilradical of $\mathfrak{q}$. We let $\tilde{\mathfrak{b}}_{0}$ be the opposite Borel subalgebra to $\mathfrak{b}_{0}$, and set $\tilde{\mathfrak{b}}=\tilde{\mathfrak{b}}_{0} \oplus \mathfrak{q}_{u}$, so $\tilde{\mathfrak{b}}$ is another Borel subalgebra of $\mathfrak{g}$. Let $\tilde{\rho}$ be the half sum of the positive roots corresponding to $\tilde{\mathfrak{b}}$. A result of Kostant in $[\mathrm{Ko}, \S 2]$ tells us that $U\left(\mathfrak{g}_{0}, e\right) \cong S(\mathfrak{t})^{W_{0}}$, where $W_{0}$ denotes the Weyl group of $\mathfrak{g}_{0}$ with respect to $\mathfrak{t}$. An explicit isomorphism

$$
\xi_{-\tilde{\rho}}: U\left(\mathfrak{g}_{0}, e\right) \stackrel{\sim}{\rightarrow} S(\mathfrak{t})^{W_{0}}
$$

is given in [BGK, Lemma 5.1], where $\xi_{-\tilde{\rho}}$ is the composition of the natural projection $U\left(\mathfrak{p}_{0}\right) \rightarrow$ $S(\mathfrak{t})$ with the shift $S_{-\tilde{\rho}}: S(\mathfrak{t}) \rightarrow S(\mathfrak{t})$.

The finite dimensional irreducible modules for $S(\mathfrak{t})^{W_{0}}$ are indexed by the set $\mathcal{L}=\mathfrak{t}^{*} / W_{0}$ of $W_{0}$-orbits in $\mathfrak{t}^{*}$. Therefore, given $\Lambda \in \mathfrak{t}^{*} / W_{0}$, we can define an irreducible $U\left(\mathfrak{g}_{0}, e\right)$-module $V_{\Lambda}$ through $\xi_{-\tilde{\rho}}$. Then we have the Verma module $M(\Lambda)$ with irreducible head $L(\Lambda)$. We note that the central character of $L(\Lambda)$ corresponds to the $W$-orbit in $\mathfrak{t}^{*}$ that contains $\Lambda$ through (3.7).

Two conjectures regarding highest weight theory for $U(\mathfrak{g}, e)$ are given in [BGK, §5.1]. The first is [BGK, Conjecture 5.2], which gives a condition for $L(\Lambda)$ to be finite dimensional. To state this conjecture we need to give some notation. We write $L(\lambda)$ for the irreducible highest weight $U(\mathfrak{g})$-module with highest weight $\lambda-\rho$, with respect to the Borel subalgebra $\mathfrak{b}$. The adjoint $G$ orbit of $e$ is denoted by $G \cdot e$ and its closure by $\overline{G \cdot e}$. Then [BGK, Conjecture 5.2] says: if $\lambda \in \Lambda$ is chosen so that $\left\langle\lambda, \alpha^{\vee}\right\rangle \notin \mathbb{Z}_{>0}$ for all $\alpha \in \Phi_{0}^{+}$, then $L(\Lambda)$ is finite dimensional if and only if $\mathcal{V} \mathcal{A}\left(\operatorname{Ann}_{U(\mathfrak{g})} L(\lambda)\right)=\overline{G \cdot e}$, where $\mathcal{V} \mathcal{A}(I)$ denotes the associated variety of an ideal $I \subset U(\mathfrak{g})$. We recall that $\Phi_{0}$ denotes the root system of $\mathfrak{g}_{0}$ with respect to $\mathfrak{t}$ and $\Phi_{0}^{+}=\Phi_{0} \cap \Phi^{+}$. 
The second conjecture is [BGK, Conjecture 5.3] which states that the category $\mathcal{O}(e ; \mathfrak{q})$ is equivalent to a certain category of generalized Whittaker modules. To define this category, we use $\tilde{\mathfrak{b}}_{u}$, the nilradical of the Borel subalgebra $\tilde{\mathfrak{b}}$ defined above.

So $\tilde{\mathfrak{b}}_{u}$ is a maximal nilpotent subalgebra of $\mathfrak{q}$, and $\chi$ restricts to a character of $\tilde{\mathfrak{b}}_{u}$. We define $\mathcal{O}(\chi, \mathfrak{q})$ to be the category of all finitely generated $U(\mathfrak{g})$-modules $M$ that are locally finite over $Z(\mathfrak{g})$ and semisimple over $\mathfrak{t}^{e}$, such that $x-\chi(x)$ acts locally nilpotently on $M$ for all $x \in \tilde{\mathfrak{b}}_{u}$. The category obtained by removing the condition that $\mathfrak{t}^{e}$ acts semisimply has been studied see for example [MS]. In $\mathcal{O}(\chi, \mathfrak{q})$ there are analogues of Verma modules that are indexed by $\mathfrak{t}^{*} / W_{0}$ and have irreducible heads. In addition to predicting an equivalence of categories $\mathcal{O}(e, \mathfrak{q}) \stackrel{\sim}{\rightarrow} \mathcal{O}(\chi, \mathfrak{q})$, BGK, Conjecture 5.3] also says that this equivalence should send $M(\Lambda)$ to the Verma module in $\mathcal{O}(\chi, \mathfrak{q})$ corresponding to $\Lambda \in \mathfrak{t}^{*} / W_{0}$. It is explained in [BGK, §5.1] that [BGK, Conjecture 5.2] is a consequence of [BGK, Conjecture 5.3].

In [Lo3, Theorem 4.1], Losev proved that a more general equivalence of categories than that predicted by [BGK, Conjecture 5.3] holds. In the proof of [Lo3, Theorem 4.1], an isomorphism $\Psi: U\left(\mathfrak{g}_{0}, e\right) \stackrel{\sim}{\rightarrow} U(\mathfrak{g}, e)_{0} / U(\mathfrak{g}, e)_{0, \sharp}$ is used, which is possibly different to $\bar{\pi}^{-1}$ from (3.3). Verma modules are defined in [Lo3] by using the isomorphism $\Psi$; however, it is remarked in [Lo4, §4.2] that $\Psi^{-1}$ may be different from $\bar{\pi}$, which means the labelling of Verma modules in [Lo3 may be different from that in [BGK]. The equivalence of categories proved in [Lo3, Theorem 4.1] does send Verma modules to Verma modules and respects labels, but the inconvenience regarding the potentially different labels of Verma modules in [Lo3] and [BGK] means that we are not able to deduce [BGK, Conjecture 5.2] immediately from [Lo3, Theorem 4.1]. The following proposition resolves this problem.

Proposition 3.12. Assume that e is of standard Levi type. Let

$$
\bar{\pi}, \Psi^{-1}: U(\mathfrak{g}, e)_{0} / U(\mathfrak{g}, e)_{0, \sharp} \stackrel{\sim}{\rightarrow} U\left(\mathfrak{g}_{0}, e\right)
$$

be the isomorphisms given by [BGK, Theorem 4.3] and [Lo3, Theorem 4.1] respectively. Then $\bar{\pi}=\Psi^{-1}$.

Proof. The composition $\bar{\pi} \circ \Psi: U\left(\mathfrak{g}_{0}, e\right) \rightarrow U\left(\mathfrak{g}_{0}, e\right)$ is an automorphism, so through the isomorphism $\xi_{-\tilde{\rho}}: U\left(\mathfrak{g}_{0}, e\right) \stackrel{\sim}{\rightarrow} S(\mathfrak{t})^{W_{0}}$ we obtain an automorphism $\sigma$ of $S(\mathfrak{t})^{W_{0}}$. From (3.7), [Lo3, Theorem 4.1(1)] and [Lo1, Theorem 1.2.2(iii)], we see that $\Psi^{-1}$ and $\bar{\pi}$ agree on the centre $Z(\mathfrak{g}, e)$ of $U(\mathfrak{g}, e)$. From (3.7) we therefore see that $\sigma$ fixes $S(\mathfrak{t})^{W} \subseteq S(\mathfrak{t})^{W_{0}}$. Thus the comorphism of $\sigma$ is a morphism $\sigma_{*}: \mathfrak{t}^{*} / W_{0} \rightarrow \mathfrak{t}^{*} / W_{0}$ that induces the identity map on $\mathfrak{t}^{*} / W$.

We define $\mathfrak{t}_{\text {reg }}^{*}=\left\{v \in \mathfrak{t}^{*} \mid\left\langle v, \alpha^{\vee}\right\rangle \neq 0\right.$ for all $\left.\alpha \in \Phi\right\}$, and let $W^{0}$ be the set of minimal length representatives of the right cosets of $W_{0}$ in $W$. Let $x \in \mathfrak{t}_{\mathrm{reg}}^{*}$ and let $X$ be the $W_{0}$-orbit of $x$. Then $\sigma_{*}(X) \in \mathfrak{t}_{\mathrm{reg}}^{*} / W_{0}$, so there exists unique $w_{x} \in W^{0}$ such that $w_{x} x \in \sigma_{*}(X)$. This gives rise to a map $f: \mathfrak{t}_{\text {reg }}^{*} \rightarrow W^{0}$ defined by $f(x)=w_{x}$. It is easy to see that $f$ is locally constant with respect to the Euclidean topology, so, since $\mathfrak{t}_{\mathrm{reg}}^{*}$ is connected, $f$ is constant, say $f(x)=w$ for all $x \in \mathfrak{t}_{\text {reg }}^{*}$.

Let $v \in W_{0}$ and $x \in \mathfrak{t}_{\mathrm{reg}}^{*}$. Then $f(x)=f(v x)=w$, so we have $w x=v^{\prime} w v x$ for some $v^{\prime} \in W_{0}$. Therefore, we see that $w v w^{-1} \in W_{0}$, so that $w \in N_{W}\left(W_{0}\right)$. Thus, conjugation by $w$ gives a map $c_{w}: \mathfrak{t}^{*} / W_{0} \rightarrow \mathfrak{t}^{*} / W_{0}$, which agrees with $\sigma_{*}$ on $\mathfrak{t}_{\text {reg }}^{*} / W_{0}$. Hence, we must have $\sigma_{*}=c_{w}$. In turn this means that $\sigma$ is the map on $S(t)^{W_{0}}$ induced by conjugation by $w$.

By [BruG, Lemma 14], there is a natural isomorphism $N_{W}\left(W_{0}\right) / W_{0} \cong N_{G^{e}}\left(\mathfrak{t}^{e}\right) / C_{G^{e}}\left(\mathfrak{t}^{e}\right)$, where $N_{G^{e}}\left(\mathfrak{t}^{e}\right)$ and $C_{G^{e}}\left(\mathfrak{t}^{e}\right)$ denote the normalizer and centralizer of $\mathfrak{t}^{e}$ in $G^{e}$ respectively. 
This isomorphism is obtained by observing that $\mathfrak{t}^{e}$ is stable under the action of $N_{W}\left(W_{0}\right)$ and $W_{0}$ fixes $\mathfrak{t}^{e}$ pointwise. Now it follows from [Lo3, Remark 5.5] that $\sigma$ fixes $\mathfrak{t}^{e} \subseteq S(\mathfrak{t})^{W_{0}}$. Hence, we must have $w=1$ and $\sigma$ is the identity map.

As a corollary we state [BGK, Conjecture 5.2], however we emphasize that [Lo3, Theorem 4.1] encapsulates the same data, however it uses the isomorphism $\Psi$ to define the analogue of $L(\Lambda)$, while to prove the results in this paper we need to use the isomorphism $\bar{\pi}$.

Corollary 3.13. Assume that $e$ is of standard Levi type. Let $\Lambda \in \mathfrak{t}^{*} / W_{0}$ and let $\lambda \in \Lambda$ be such that $\left\langle\lambda, \alpha^{\vee}\right\rangle \notin \mathbb{Z}_{>0}$ for all $\alpha \in \Phi_{0}^{+}$. Then $L(\Lambda)$ is finite dimensional if and only if $\mathcal{V} \mathcal{A}\left(\operatorname{Ann}_{U(\mathfrak{g})} L(\lambda)\right)=\overline{G \cdot e}$.

We finish this subsection by discussing Proposition 3.6 in the case where $e$ is of standard Levi type. Let $\mathfrak{s}$ be a full subalgebra of $\mathfrak{t}^{e}$. For $\Lambda \in \mathcal{L}=\mathfrak{t}^{*} / W_{0}$, we define $V_{\Lambda}$ and the Verma module $M^{\mathfrak{s}}(\Lambda)=M^{\mathfrak{s}}\left(V_{\Lambda}\right)$ for $U\left(\mathfrak{g}^{\mathfrak{s}}, e\right)$ as above using the isomorphism $\xi_{-\tilde{\rho}}: U\left(\mathfrak{g}_{0}, e\right) \stackrel{\sim}{\rightarrow} S(\mathfrak{t})^{W_{0}}$ from (3.11). Note that we use the shift by $-\tilde{\rho}$ rather than $-\tilde{\rho}_{s}$, where $\tilde{\rho}_{\mathfrak{s}}$ is the analogue of $\tilde{\rho}$ for $\mathfrak{g}^{\mathfrak{s}}$; this can be viewed as a "shift in origin" as $\tilde{\rho}-\tilde{\rho}_{\mathfrak{s}}$ is orthogonal to all roots in $\Phi^{\mathfrak{s}}$. With this convention Proposition 3.6 for the standard Levi case is tidily stated as:

Corollary 3.14. Assume that e is of standard Levi type. Let $\Lambda \in \mathfrak{t}^{*} / W_{0}$ and suppose that $L(\Lambda)$ is finite dimensional. Then $L^{\mathfrak{s}}(\Lambda)$ is finite dimensional.

3.6. Component group action. Let $\mathfrak{s}$ be a full subalgebra of $\mathfrak{t}^{e}$ and let $\tilde{G}^{\mathfrak{s}}$ be the centralizer of $\mathfrak{s}$ in $\tilde{G}$. As discussed at the end of $₫ 2.2$, there is an action of the component group $\tilde{C}^{\mathfrak{s}}=\tilde{G}^{\mathfrak{s}, e} /\left(\tilde{G}^{\mathfrak{s}, e}\right)^{\circ}$ on the set of finite dimensional irreducible $U\left(\mathfrak{g}^{\mathfrak{s}}, e\right)$-modules; here $\tilde{G}^{\mathfrak{s}, e}$ denotes the centralizer of $e$ in $\tilde{G}^{\mathfrak{s}}$.

Given $c \in \tilde{C}^{\mathfrak{s}}$ and an irreducible $U\left(\mathfrak{g}^{\mathfrak{s}}, e\right)$-module $L$, we write $c \cdot L$ for the irreducible $U\left(\mathfrak{g}^{\mathfrak{s}}, e\right)$-module obtained by twisting with $c$. By definition $c \cdot L$ is equal to $L$ as a vector space with action given by choosing $\dot{c}$ in $\tilde{G}^{\mathfrak{s}, e}$ that lifts $c$ and setting $u \cdot v=(\dot{c} \cdot u) v$ for $u \in U\left(\mathfrak{g}^{\mathfrak{s}}, e\right)$ and $v \in L$; this only depends on the choice of $\dot{c}$ up to isomorphism. This gives rise to an action of $\tilde{C}^{\mathfrak{s}}$ on $\mathcal{L}^{\mathfrak{s},+}$ : for $c \in \tilde{C}^{\mathfrak{s}}$ and $\Lambda \in \mathcal{L}^{\mathfrak{s},+}$ we write $c \cdot{ }_{\mathfrak{s}} \Lambda$ for the image of $\Lambda$ under $c$; by definition we have $c \cdot L^{\mathfrak{s}}(\Lambda) \cong L^{\mathfrak{s}}\left(c \cdot_{\mathfrak{s}} \Lambda\right)$. Moreover, as the action of $\tilde{C}^{\mathfrak{s}}$ fixes $Z\left(\mathfrak{g}^{\mathfrak{s}}, e\right)$, we get an action on $\mathcal{L}_{\psi}^{\mathfrak{s},+}$ for each $\psi: Z\left(\mathfrak{g}^{\mathfrak{s}}\right) \rightarrow \mathbb{C}$. In the case $\mathfrak{s}=0$ we omit the subscript in the notation for the action of $\tilde{C}$ on $\mathcal{L}^{+}$.

The inclusion $\tilde{G}^{\mathfrak{s}, e} \hookrightarrow \tilde{G}^{e}$ induces a injective map $\iota: \tilde{C}^{\mathfrak{s}} \rightarrow \tilde{C}$, so we can view $\tilde{C}^{\mathfrak{s}}$ as a subgroup of $\tilde{C}$. We briefly explain why $\iota$ is injective. We can also induce $\iota$ from the inclusion $\tilde{H}^{\mathfrak{s}, e} \hookrightarrow \tilde{H}^{e}$. If $x \in \tilde{H}^{\mathfrak{s}, e} \cap\left(\tilde{H}^{e}\right)^{\circ}$, then $x$ and $S$ generate a connected Abelian subgroup of $\tilde{H}^{e}$, where $S$ is the torus in $\tilde{G}$ with Lie algebra $\mathfrak{s}$. Therefore, there is a Borel subgroup of $D$ of $\left(\tilde{H}^{e}\right)^{\circ}$ containing $x$ and $S$. Then the centralizer of $\mathfrak{s}$ in $D$, denoted $D^{\mathfrak{s}}$, is a Borel subgroup of $\left(\tilde{H}^{\mathfrak{s}, e}\right)^{\circ}$ and $x \in D^{\mathfrak{s}}$. Hence, $x \in\left(\tilde{H}^{\mathfrak{s}, e}\right)^{\circ}$.

By Proposition 3.6, we have $\mathcal{L}^{+} \subseteq \mathcal{L}^{\mathfrak{s},+}$. Therefore, given $\Lambda \in \mathcal{L}^{+}$and $c \in \tilde{C}^{\mathfrak{s}} \subseteq \tilde{C}$ we can consider $c \cdot{ }_{\mathfrak{s}} \Lambda$ and $c \cdot \Lambda$. The following lemma says that these two actions of $c$ on $\Lambda$ are equal.

Lemma 3.15. Let $c \in \tilde{C}^{\mathfrak{s}}$ and $\Lambda \in \mathcal{L}^{+}$. Then $c \cdot{ }_{\mathfrak{s}} \Lambda=c \cdot \Lambda$.

Proof. Consider $c \cdot L_{\mathfrak{s}}\left(L^{\mathfrak{s}}(\Lambda)\right)$ and view $N=\left(1+U(\mathfrak{g}, e)_{\mathfrak{s}, \sharp}\right) \otimes L^{\mathfrak{s}}(\Lambda)$ as a subspace. Then $N$ is a maximal $\mathfrak{s}$-weight space of $c \cdot L_{\mathfrak{s}}\left(L^{\mathfrak{s}}(\Lambda)\right)$ because $c \in \tilde{C}^{\mathfrak{s}}$. Therefore, we can view $N$ as a $U\left(\mathfrak{g}^{\mathfrak{s}}, e\right)$-module, and as such it is isomorphic to $c \cdot L^{\mathfrak{s}}(\Lambda)$, which by definition is isomorphic to $L^{\mathfrak{s}}\left(c \cdot{ }_{\mathfrak{s}} \Lambda\right)$. 
Therefore, there is a homomorphism from the submodule of $c \cdot L_{\mathfrak{s}}\left(L^{\mathfrak{s}}(\Lambda)\right)$ generated by $N$ to $L_{\mathfrak{s}}\left(L^{\mathfrak{s}}\left(c \cdot{ }_{\mathfrak{s}} \Lambda\right)\right)$. Now using the fact that $c \cdot L_{\mathfrak{s}}\left(L^{\mathfrak{s}}(\Lambda)\right)$ is irreducible, we see that it must be isomorphic to $L_{\mathfrak{s}}\left(L^{\mathfrak{s}}(c \cdot \mathfrak{s} \Lambda)\right)$.

Using Proposition [3.6, we have $c \cdot L(\Lambda) \cong c \cdot L_{\mathfrak{s}}\left(L^{\mathfrak{s}}(\Lambda)\right) \cong L\left(c \cdot{ }_{\mathfrak{s}} \Lambda\right)$ and by definition $c \cdot L(\Lambda) \cong L(c \cdot \Lambda)$, so we are done.

\section{Combinatorics of tables}

Our proof of Theorem 5.13 depends on combinatorics of the symmetric pyramid associated to a nilpotent element of a classical Lie algebra as described in Section 5. In this section, we present the underlying combinatorial results that we require for the proof.

Throughout the remainder of this article, by a partition we mean a multiset of positive integers; we usually denote partitions by writing them as a sequence in either increasing or decreasing order, and often use exponential notation to denote repeated entries. We write $>$ for the usual dominance ordering on partitions. Given a partition $\mathbf{p}$ we write $\mathbf{p}^{T}$ for the transpose partition. We recall the elementary fact that if $\mathbf{p}$ and $\mathbf{q}$ are partitions of the same integer with $\mathbf{p} \geq \mathbf{q}$, then $\mathbf{p}^{T} \leq \mathbf{q}^{T}$.

4.1. Frames and tables. We define a frame to be a connected arrangement of boxes in the plane such that the boxes are aligned in rows, and so that rows are connected. A frame is called justified if the boxes are aligned in columns and is called left justified if its rows all start in the same column; so a left justified frame is justified. We say a justified frame $F$ is preconvex if given any two columns in $F$, we can slide one of them horizontally so that it fits entirely inside the other. We say a frame is convex if it is preconvex and it is has connected columns. For example

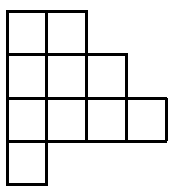

is a left justified convex frame, and

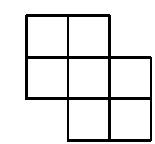

is a frame that is justified, but not left justified and not convex. We note that a left justified frame is convex if and only if its columns are connected.

A frame filled with integers is called a table. Given a table $A$, the frame of $A$ is obtained by removing the integers in the boxes. We say a table is justified, left justified or convex if its frame is.

Let $F$ be a frame. We write $\operatorname{Tab}(F)$ for the set of all tables with frame $F$. The row equivalence class of $A \in \operatorname{Tab}(F)$ is obtained by taking all possible permutations of the entries in the rows of $A$; we write $\bar{A}$ for the row equivalence class of $A$. We define $\operatorname{Row}(F)=$ $\{\bar{A} \mid A \in \operatorname{Tab}(F)\}$. Given $A \in \operatorname{Tab}(F)$, there is a unique element $A \leq \in \bar{A}$ with weakly increasing rows. Let $\operatorname{Tab}^{\leq}(F)=\{A \leq \mid A \in \operatorname{Tab}(F)\}$, which is in bijection with $\operatorname{Row}(F)$.

Given a frame $F$ we denote by $l(F)$ the left justified frame obtained from $F$ by justifying the rows. Given $A$ in $\operatorname{Tab}(F)$ we also define $l(A) \in \operatorname{Tab}(l(F))$ by left justifying all of the rows of $A$. 
Suppose $F$ is justified. We say $A \in \operatorname{Tab}(F)$ is column strict if the columns of $A$ are decreasing and we say that $A$ is row equivalent to column strict if there exists column strict $B \in \bar{A}$. For example,

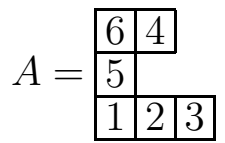

is column strict, but $A \leq$ is not column strict, so $A \leq$ is row equivalent to column strict. We emphasize that in our definition of column strict we require entries in columns to be decreasing over gaps in columns. So for example

\begin{tabular}{|l|l|}
\hline 5 & 1 \\
\hline 4 & \multicolumn{1}{|l}{} \\
\hline 2 & 3 \\
\hline
\end{tabular}

is neither column strict nor row equivalent to column strict. For a general frame $F$, we say that $A \in \operatorname{Tab}(F)$ is justified row equivalent to column strict if $l(A)$ is row equivalent to column strict. We note that in the case $F$ is preconvex, then the notions of row equivalent to column strict and justified row equivalent to column strict coincide.

Let $A \in \operatorname{Tab}(F)$. We denote the number of rows of $F$ by $r_{F}=r_{A}$ and label the rows of $F$ and $A$ with $1, \ldots, r_{F}$ from top to bottom. We define $\operatorname{part}(F)=\operatorname{part}(A)$ to be the partition given by the row lengths in $F$. For example, if

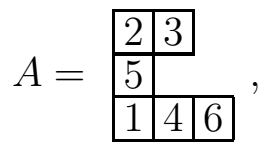

then $\operatorname{part}(A)=(3,2,1)$. If $\operatorname{part}(F)=\mathbf{p}$, then we sometimes say $F$ is associated to $\mathbf{p}$. Given $1 \leq i<j \leq r_{A}$, we write $A_{i}$ for the $i$ th row of $A$, and $A_{j}^{i}$ for the table obtained from $A$ by removing rows $1, \ldots, i-1$ and $j+1, \ldots, r_{A}$. Note that when considering subtables of the form $A_{j}^{i}$ we continue to use the labelling of rows inherited from $A$. Let word $(A)$ denote the sequence of integers created by listing the entries in $A$ row by row from left to right, top to bottom. With $A$ as above, we have word $(A)=(2,3,5,1,4,6)$.

A tableaux is a left justified table $A$ with increasing row lengths such that $A=A \leq$ and $A$ is column strict. For example

$$
\begin{aligned}
& \begin{array}{|l|l|}
\hline 5 & 7 \\
\hline
\end{array} \\
& \begin{array}{ll}
\hline 2 & 4 \\
\hline
\end{array} \\
& \begin{array}{|l|l|l|}
\hline 1 & 3 & 6 \\
\hline
\end{array}
\end{aligned}
$$

is a tableaux.

4.2. The Robinson-Schensted algorithm. Our discussion of the Robinson-Schensted algorithm follows $[\mathrm{F}]$. The Robinson-Schensted algorithm takes as input a finite list of integers, called a word, and outputs a tableaux.

The algorithm is defined recursively, starting with the empty tableaux. If $w=a_{1} \ldots a_{n}$ is the word, then we assume that $a_{1}, \ldots, a_{i-1}$ have already been inserted. To insert $a_{i}$, assume $b_{1} \leq \cdots \leq b_{k}$ is the bottom row of the tableaux. If $a_{i} \geq b_{k}$, then insert $a_{i}$ at the end of the bottom row. Otherwise there exists $j$ such that $a_{i}<b_{j}$ and $a_{i} \geq b_{j-1}$. Replace $b_{j}$ with $a_{i}$, then recursively insert $b_{j}$ into the diagram with the bottom row removed. In this 
latter situation we say that $a_{i}$ bumps $b_{j}$. We write $\operatorname{RS}(w)$ for the output of the RobinsonSchensted algorithm applied to the word $w$. Given a frame $F$ and $A \in \operatorname{Tab}^{\leq}(F)$ we write $\mathrm{RS}(A)=\mathrm{RS}(\operatorname{word}(A))$.

An important related concept is that of Knuth equivalence on the set of words of integers. If $x, y$, and $z$ are integers for which $x<y<z$, and $u$ and $v$ are words of integers, then we declare that

$$
u y z x v \equiv u y x z v
$$

and

$$
u x z y v \equiv u z x y v
$$

The equivalence relation on the set of words of integers generated by (4.1) and (4.2) is the Knuth equivalence relation. The Robinson-Schensted algorithm can be interpreted as choosing a canonical representative in the Knuth equivalence class of a word. It is proved in [F, §2] that if $w$ is a word of integers, then $w \equiv \operatorname{word}(\operatorname{RS}(w))$.

We now discuss two alternative methods for calculating part $(\operatorname{RS}(w))$, which are dual to each other. The first involves finding disjoint weakly increasing subwords of $w$. We define $\ell(w, k)$ to be the maximum possible sum of the lengths of $k$ disjoint weakly increasing subsequences of $w$. For example, $\ell(412563,1)=4$ use the increasing subword 1256 and $\ell(412563,2)=6$ using the subwords 456 and 123. The following lemma is an immediate corollary of [F, Lemma 3.1.1] and [F, Lemma 3.1.2]:

Lemma 4.3. Let $w$ be a word of integers and let $\mathbf{p}=\left(p_{1} \geq \cdots \geq p_{n}\right)=\operatorname{part}(\operatorname{RS}(w))$. Then for all $k \geq 1, \ell(w, k)=p_{1}+\cdots+p_{k}$.

The dual version of the above lemma considers lengths of strictly decreasing subwords. We define $\mathrm{s}(w, k)$ to be the maximum possible sum of the lengths of $k$ disjoint strictly decreasing subsequences of $w$. The following lemma is a consequence of [Gr, Theorem 1.6].

Lemma 4.4. Let $w$ be a word of integers and let $\mathbf{p}^{T}=\left(p_{1}^{*} \geq \cdots \geq p_{n}^{*}\right)$ be the dual partition to $\mathbf{p}=\operatorname{part}(\operatorname{RS}(w))$. Then for all $k \geq 1,6(w, k)=p_{1}^{*}+\cdots+p_{k}^{*}$.

Remark 4.5. As the above example illustrates, if the first $k$ terms of $\operatorname{part}(\operatorname{RS}(w))$ are $p_{1}, \ldots, p_{k}$, it does not mean that we can find $k$ disjoint weakly increasing subsequences of $w$ of lengths $p_{1}, \ldots, p_{k}$. However, one situation where this is possible is when $\operatorname{part}(\operatorname{RS}(w))$ is of the form $\left(p_{1}^{a} \geq\left(p_{1}-1\right)^{b} \geq \ldots\right)$. In this case it is easy to see that one must be able to find $a$ disjoint weakly increasing subsequences of $w$ length $p_{1}$, which are disjoint from $b$ disjoint weakly increasing subsequences of $w$ of length $p_{1}-1$.

The situation where we consider $\mathbf{p}^{T}$ and strictly decreasing subsequences is completely analogous.

4.3. Column strict tables. The following theorem, which is proved after three preliminary lemmas, is the important combinatorial result required for the proof of Theorem 5.13 .

Theorem 4.6. Suppose $F$ is convex and let $A \in \operatorname{Tab}^{\leq}(F)$. Then $\operatorname{part}(\operatorname{RS}(A))=\operatorname{part}(F)$ if and only if $A$ is row equivalent to column strict.

An important part of the proof of Theorem 4.6 involves the notion of row swapping. Fix a justified preconvex frame $F$, let $A \in \operatorname{Tab}^{\leq}(F)$ and let $1 \leq i<r_{F}$. We define $s_{i}(F)$ to be the frame obtained from $F$ by swapping the $i$ th and $(i+1)$ th row; note that $F$ being preconvex 
ensures that $s_{i}(F)$ is connected. Below we define $s_{i}(A) \in \operatorname{Tab}^{\leq}\left(s_{i}(F)\right)$ provided $A_{i}$ and $A_{i+1}$ satisfy certain conditions.

Let $c_{1} \leq c_{2} \leq \ldots c_{s}$ be the entries of $A_{i}$ and let $d_{1} \leq d_{2} \leq \ldots d_{t}$ be the entries of $A_{i+1}$. We split into two cases.

Case 1: $s<t$. Then $s_{i}(A)$ is defined if $d_{i}<c_{i}$ for $i=1, \ldots, s$. In this case, we choose $e_{1}, \ldots, e_{s}$ from $d_{1}, \ldots d_{t}$ so that $e_{i}<c_{i}$ and $\sum_{i=1}^{s} c_{i}-e_{i}$ is minimal. Then $e_{1}, \ldots e_{s}$ form the entries of row $i+1$ in $s_{i}(A)$, while the remaining entries in $A_{i+1}$ are added to $c_{1}, \ldots, c_{s}$ (and rearranged into weakly increasing order) to form the entries of row $i$ in $s_{i}(A)$.

Case 2: $s>t$. Then $s_{i}(A)$ is defined if $d_{s+1-i}<c_{t+1-1}$ for $i=1, \ldots, t$. In this case, we choose $e_{1}, \ldots, e_{t}$ from $c_{1}, \ldots c_{s}$ so that $e_{i}>d_{i}$ and $\sum_{i=1}^{t} e_{i}-d_{i}$ is minimal. Then $e_{1}, \ldots, e_{t}$ form the entries of row $i$ in $s_{i}(A)$, while the remaining elements from row $i$ are added to $d_{1}, \ldots, d_{t}$ (and rearranged into weakly increasing order) to form the entries of row $i+1$ in $s_{i}(A)$.

For example we can apply row swapping to rows 2 and 3 of

$$
A=\begin{array}{|l|l|l|}
\hline 6 & 7 & \\
\hline 3 & 5 & \\
\hline 1 & 2 & 4 \\
\hline
\end{array}
$$

and we get

$$
s_{2}(A)=\begin{array}{l|l|l}
\hline 6 & 7 & \\
\hline 1 & 3 & 5 \\
\hline 2 & 4 &
\end{array} .
$$

The next lemma is an easy observation about the above definition, so we omit the proof.

Lemma 4.7. Let $A \in \mathrm{Tab}^{\leq}(F)$ and suppose that $s_{i}(A)$ is defined. Then $s_{i}\left(s_{i}(A)\right)$ is defined and equal to $A$.

Our next lemma relates row swapping to the Robinson-Schensted algorithm.

Lemma 4.8. Let $A \in \mathrm{Tab}^{\leq}(F)$, let $1 \leq i<r_{A}$, and suppose $s_{i}(A)$ is defined. Then $\operatorname{RS}(A)=\operatorname{RS}\left(s_{i}(A)\right)$.

Proof. It is easy to check that, in Case 2 above, $\operatorname{RS}\left(A_{i+1}^{i}\right)$ is precisely $s_{i}(A)_{i+1}^{i}$. It follows that $\operatorname{word}(A)$ is Knuth equivalent to word $\left.\left(s_{i}(A)\right)\right)$, so that $\operatorname{RS}(A)=\operatorname{RS}\left(s_{i}(A)\right)$.

In order to deal with Case 1 above, we use Lemma 4.7 and apply the result for Case 2 to $s_{i}(A)$.

The following is the main technical lemma required for the proof of Theorem 4.6.

Lemma 4.9. Let $F$ be preconvex, and let $A \in \mathrm{Tab}^{\leq}(F)$ be such that $A$ is row equivalent to column strict.

(i) Suppose also that $A_{i+1}^{1}$ is convex, and the length of row $i$ is greater than the length of row $i+1$. Then $s_{i}(A)$ is defined and is row equivalent to column strict.

(ii) Suppose also that $A_{r_{A}}^{i}$ is convex, and the length of row $i$ is less than the length of row $i+1$. Then $s_{i}(A)$ is defined and is row equivalent to column strict.

Proof. We only prove part (i) as the proof of part (ii) is very similar. We can assume $F$ is left justified because $A$ is row equivalent to column strict if and only if $l(A)$ is.

We first prove the lemma in the case that row $i+1$ has 1 box and row $i$ has 2 boxes. Let $\tilde{A} \in \bar{A}$ be column strict. Denote the entries in column 1 of $\tilde{A}$ by $a_{1}, \ldots, a_{r(A)}$ and the entries 
in column 2 of $\tilde{A}$ above row $i$ by $b_{j}, \ldots, b_{i}$, where $1 \leq j \leq i, b_{j-1}$ does not exist, and $a_{k}, b_{k}$ lie in row $k$. Since $A_{i+1}^{1}$ is convex we have that $b_{k}$ is defined for all $k$ between $j$ and $i$.

As $\tilde{A}$ is column strict we have $a_{i+1}<a_{i}$, which implies that $s_{i}(A)$ is defined. Also we have that $s_{i}(A)_{i+1}^{i}$ is $\operatorname{RS}\left(A_{i+1}^{i}\right)$, so we consider applying the Robinson-Schensted algorithm to $\operatorname{word}\left(A_{i}^{i+1}\right)$.

If $a_{i+1}$ bumps $a_{i}$, then it is easy to see that $s_{i}(A)$ is obtained from $A$ by simply moving the box containing $b_{i}$ down to row $i+1$. Then define $\tilde{A}^{\prime} \in \operatorname{Row}\left(s_{i}(A)\right)$ to be obtained from $\tilde{A}$ by moving the box containing $b_{i}$ down to row $i+1$. It is clear that $\tilde{A}^{\prime}$ is column strict meaning that $s_{i}(A)$ is row equivalent to column strict.

If $a_{i+1}$ bumps $b_{i}$, then we generate a new column strict table $\hat{A} \in \bar{A}$ as follows. First swap the boxes containing $a_{i}$ and $b_{i}$. The resulting diagram is now column strict except $a_{i}$ may be larger than $b_{i-1}$. If this occurs, then also swap the boxes containing $a_{i-1}$ and $b_{i-1}$. Again the resulting diagram is column strict, except $a_{i-1}$ may be larger than $b_{i-2}$. If this occurs, then swap the boxes containing $a_{i-2}$ and $b_{i-2}$. We keep repeating this process until we obtain a column strict diagram, which must eventually happen, as $b_{j-1}$ does not exist. Now the resulting diagram satisfies the first case, that is the new $a_{i+1}$ bumps the new $a_{i}$, so $s_{i}(A)$ is row equivalent to column strict.

Next we consider the general case. Let $\tilde{A} \in \operatorname{Row}(A)$ be column strict, and denote the entries of row $i$ of $\tilde{A}$ by $c_{1}, \ldots, c_{r}$ and the entries of row $i+1$ of $\tilde{A}$ by $d_{1}, \ldots, d_{s}$. Suppose that when applying the Robinson-Schensted algorithm to $\operatorname{word}\left(A_{i+1}^{i}\right)$, there exists $j, t$ such that $1 \leq j \leq s$ and $t>s$, and such that $d_{j}$ bumps $c_{t}$. Then we form another table $\hat{A} \in \bar{A}$ as follows. We begin by swapping the boxes containing $c_{t}$ and $c_{j}$ in $\tilde{A}$. The resulting diagram is column strict except that the entry in the $(i-1)$ th row and the th column may not be greater than $c_{j}$. We can continue to swap boxes between the $j$ th and th column, as in the above case to obtain $\hat{A}$. Now in $\hat{A}$ one more of the first $s$ boxes of the $i$ th row is bumped during the application of the Robinson-Schensted algorithm to word $\left(A_{i+1}^{i}\right)$ than is the case for $\tilde{A}$. It follows that we can assume, by induction, that $c_{1}, \ldots, c_{s}$ are the elements which are bumped in the Robinson-Schensted algorithm. In this situation, we can define $\tilde{A}^{\prime} \in \overline{\left(s_{i}(A)\right)}$ to be obtained from $\tilde{A}$ by simply moving the boxes containing $c_{s+1}, \ldots, c_{r}$ down to row $i+1$. It is clear that $\tilde{A}^{\prime}$ is column strict, so that $s_{i}(A)$ is row equivalent to column strict.

Lemma 4.10. Let $A \in \mathrm{Tab}^{\leq}(F)$ and $1 \leq i<r_{A}$, and suppose that $\operatorname{part}(\operatorname{RS}(A))=\operatorname{part}(F)$. Then $s_{i}(A)$ is defined.

Proof. Let $\mathbf{p}=\left(p_{1} \geq \cdots \geq p_{n}\right)=\operatorname{part}(F)$. Let $p_{j}$ be the length of $A_{i}$ and $p_{k}$ be the length of $A_{i+1}$. We assume the $p_{j} \geq p_{k}$, the other case being entirely similar. Suppose $s_{i}(A)$ is undefined. Then it is easy to see that there is an increasing subsequence in $\operatorname{word}\left(A_{i+1}^{i}\right)$ of length greater than $p_{j}$. This implies that $\ell(\operatorname{word}(A), j)>p_{1}+\cdots+p_{j}$, so that $\operatorname{part}(\mathrm{RS}(A))>$ $\operatorname{part}(F)$ by Lemma 4.3 .

We are now in a position to prove Theorem 4.6.

Proof of Theorem 4.6. First we prove by induction on $r_{A}$ (the number of rows of $A$ ) that if $A$ is row equivalent to column strict, then we can perform a sequence of row swaps $s_{i_{1}}, \ldots, s_{i_{m}}$ so that $B=s_{i_{1}} \ldots s_{i_{m}}(A)$ is defined and satisfies:

(i) $B$ has increasing row lengths; and 
(ii) $B$ is row equivalent to column strict.

The case where $r_{A}=0$ is trivial. If $A_{1}$ is a row of shortest length, then it is clear that we can form a sequence of row swaps satisfying the above conditions for $A$ if and only if we can do so for $A_{r_{A}}^{2}$ in which case we can apply induction. So we assume that $A_{r_{A}}$ is of shortest length. In this case, let $k$ be minimal such that $A_{k+1}, \ldots, A_{r_{A}}$ have the same length. Then clearly $A_{k+1}^{1}$ is convex. Therefore, we can apply Lemma 4.6 to see that $s_{k}(A)$ is defined and is row equivalent to column strict. We continue by applying the row swapping operations $s_{k-1}, \ldots, s_{1}$ in turn. Inductively, we see that the table $A(j)=s_{j} \ldots s_{k}(A)$ is defined and row equivalent to column strict by Lemma 4.6. Now $A(1)_{1}$ is a row of shortest length in $A(1)$, so we are now in the case above where we can apply induction. Hence, we can find the desired sequence of row swaps.

Suppose that $A$ is row equivalent to column strict and let $B$ be the table obtained from $A$ by a sequence of row swaps and satisfying (i) and (ii) above. Then in fact $B$ must be column strict. Now it is a straightforward exercise to check that $\operatorname{RS}(B)=B$ so that $\operatorname{part}(\operatorname{RS}(B))=\operatorname{part}(F)$. Hence using Lemma 4.8, we have $\operatorname{part}(\operatorname{RS}(A))=\operatorname{part}(F)$ as required.

Now assume that part $(\operatorname{RS}(A))=\operatorname{part}(F)$. An inductive argument very similar to above shows that we can perform a sequence of row swaps $s_{i_{1}}, \ldots, s_{i_{m}}$ so that $B=s_{i_{1}} \ldots s_{i_{m}}(A)$ is defined and satisfies:

(i) $B$ has increasing row lengths; and

(ii) $\operatorname{part}(\operatorname{RS}(B))=\operatorname{part}(F)$.

The only adaptation required is to use Lemma 4.10, to see that the row swaps are defined and Lemma 4.8 to see that they preserve the output of the Robinson-Schensted algorithm. We can use Lemma 4.4 to see that $B$ must be column strict. Now by performing all of the row swapping operations in reverse and applying Lemma 4.9, we see that $A$ is row equivalent to column strict.

Suppose $F$ is convex and let $\bar{A} \in \operatorname{Row}(F)$. Below we explain a process to determine if there exists column strict $B \in \bar{A}$ and to find such $B$ if it exists.

Algorithm 4.11. Suppose $F$ is convex and let $\bar{A} \in \operatorname{Row}(F)$. Let $a_{1} \leq a_{2} \leq \cdots \leq a_{m}$ be all the entries of $A$. We proceed in steps, after the first $(i-1)$ steps we have inserted $a_{1}, \ldots, a_{i-1}$ in $F$.

ith step: We consider $a_{i}$ and suppose that it lies in row $j_{i}$. We consider all empty boxes $b$ in row $j_{i}$ for which either there is no box below $b$ or the box below $b$ has already been filled.

If no such box exists, then we output that $A$ is not row equivalent to column strict and finish. Otherwise, from all such $b$ we choose the one with the most boxes above it, and the rightmost one if there is more than one such $b$. We insert $a_{i}$ in to this box.

After the $m$ th step a column strict element of $\bar{A}$ is output.

Before we argue that this algorithm is correct, we illustrate it with an example. We consider

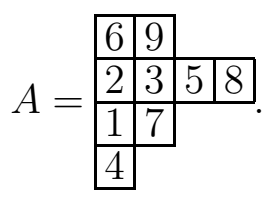


By applying Algorithm 4.11, we get the following sequence
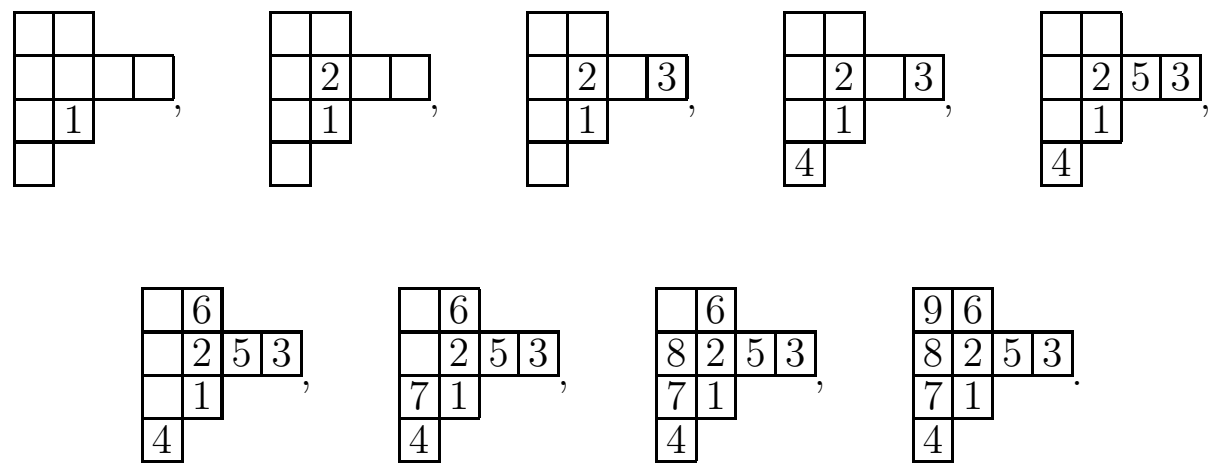

Proof of correctness of Algorithm 4.11. We show by induction on $i$ that if there exists column strict $B \in \bar{A}$, then there is such $B$ with boxes as filled by the first $i$ steps of the algorithm. The case $i=0$ is trivial.

Consider the $i$ th step. If it is not possible to find an empty box $b$ in row $j_{i}$ for which either there is no box below $b$ or the box below $b$ has already been filled, then it is clear that $A$ cannot be row equivalent to column strict. Now suppose that $A$ is row equivalent to column strict and that $B \in \bar{A}$ is column strict. By induction we may assume that boxes in $B$ containing $a_{1}, \ldots, a_{i-1}$ are filled as in the first $i-1$ steps. Let $b$ be the box that the algorithm says to put $a_{i}$ in, and let $b^{\prime}$ be the box in $B$ containing $a_{i}$. Now as in the proof of Lemma 4.9, we may swap entries of $B$ in the columns containing $b$ and $b^{\prime}$ to obtain column strict $B^{\prime} \in \bar{A}$ with $a_{i}$ in $b$. This completes the induction.

4.4. s-frames and s-tables. To manage the data associated to nilpotent elements in classical Lie algebras in the next section we require a symmetric version of frames and tables. We define an s-frame to be a frame where the boxes, are arranged symmetrically around a central point. For this paper, we only consider s-frames with an even number of rows. We say that an s-frame is a symmetric pyramid if the row lengths increase from the centre outwards; we note that a symmetric pyramid is uniquely determined by its row lengths.

An example of an s-frame (which is not a symmetric pyramid) is

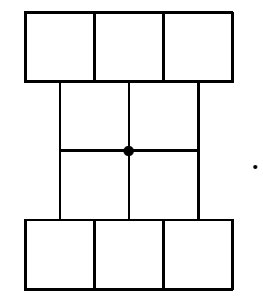

We define an $s$-table to be an s-frame filled with integers skew-symmetrically with respect to the centre. Given an $s$-frame $F$, we write $\operatorname{sTab}(F)$ for the set of s-tables with frame $F$. We write $\bar{A}^{s}=\bar{A} \cap \operatorname{sTab}(A)$ for the set of s-tables row equivalent to $A$. The subset of $\operatorname{sTab}(F)$ consisting of s-tables with entries weakly increasing along rows is denoted by $\mathrm{sTab}^{\leq}(F)$. For example 


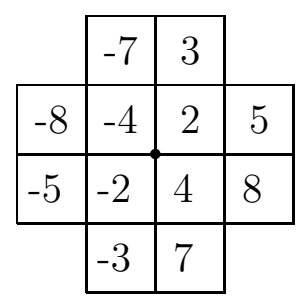

is an s-table, which lies in $\mathrm{sTab}^{\leq}(F)$, where $F$ is its s-frame.

Let $F$ be an s-frame and $A \in \operatorname{sTab}(F)$. By assumption, $F$ has an even number of rows, say $2 r$. We label the rows of $F$ and $A$ with $-r, \ldots,-1,1, \ldots, r$ from top to bottom. Given $i= \pm 1, \ldots, \pm r$ we write $A_{i}$ for row of $A$ labelled by $i$, and for $i>0$ we write $A_{i}^{-i}$ for the s-table obtained by removing rows $\pm 1, \ldots, \pm(i-1)$. The table obtained from $A$ by removing all boxes below the central point is denoted by $A^{+}$. For example if $A$ is the table above, then

$$
A^{+}=\begin{array}{|l|l|l|l|}
\cline { 2 - 3 } & -7 & 3 & \multicolumn{1}{|l}{} \\
\hline-8 & -4 & 2 & 5 \\
\hline
\end{array}
$$

In case the row lengths of $A$ all have the same parity, then $A$ is justified, thus there is a natural notion of $A$ being row equivalent to column strict as an s-table. In the next lemma we see that in fact this is not a stronger requirement than being row equivalent to column strict.

Lemma 4.13. Let $F$ be an s-frame such all row lengths in $F$ have the same parity and $A \in \operatorname{sTab}(F)$. Then there exists column strict $B \in \bar{A}^{s}$ if and only if $A$ is row equivalent to column strict.

Proof. The only if part is trivial. Suppose that $A$ is row equivalent to column strict, then we explain how we can adapt Algorithm 4.11 to find $B \in \bar{A}^{s}$, which is column strict.

We write $a_{1}>\cdots>a_{m}$ for the positive entries in $A$. Then in the $i$ th step of our adaptation, we insert the entries $\pm a_{i}$. To insert $-a_{i}$ we follow the rules in Algorithm 4.11 and to insert $a_{i}$ we look for the empty box which is leftmost such that there is no box above it or the box above it has already been filled, and has as many empty boxes below it as possible. (So we are mixing Algorithm 4.11 with its "dual" version.)

The proof of correctness of Algorithm 4.11 can be easily modified to show that this adapted version does insert entries in to all the boxes in $F$. It is clear that the resulting table $B$ is an s-table. So $B \in \bar{A}^{s}$ is column strict, as required.

Last in this subsection we generalize the row swapping procedure to s-tables. As above let $F$ be an s-frame with $2 r$ rows, and let $A \in \mathrm{Tab}^{\leq}(F)$. Let $i=1, \ldots, r-1$. We can define the row swapping operation $s_{i}$ as before, so that it swaps rows $i$ and $i+1$. Using the same rules we can define the row swapping operation $s_{-i}$ that swaps rows $-(i+1)$ and $-i$. Now we define the operator $\bar{s}_{i}$, on sTab $\leq(F)$, to be the composition of $s_{i}$ and $s_{-i}$. We note that $s_{i}(A)$ is defined if and only if $s_{-i}(A)$ is defined, and that the operators $s_{i}$ and $s_{-i}$ commute. If $s_{i}(A)$ is undefined, then we say $\bar{s}_{i}$ is undefined on $A$. Also we note that when $s_{i}$ is defined, then the action of $s_{-i}$ is "dual" to that of $s_{i}$, so $\bar{s}_{i}(A)$ is an s-table. An example of a row 
swapping operation $\bar{s}_{i}$ is

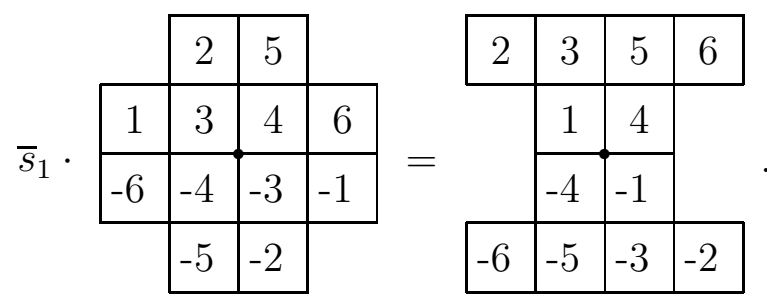

Remark 4.15. When considering orthogonal Lie algebras in Section 5, we need to consider tables where the entries are elements of $\mathbb{Z}+\frac{1}{2}$. Given a s-frame $F$, we define $\operatorname{sTab}_{\frac{1}{2}}(F)$ to be the set of all skew-symmetric fillings of $F$ by elements of $\mathbb{Z}+\frac{1}{2}$. We also define $\operatorname{sTab}_{+}(F)=\operatorname{sTab}(F) \cup \operatorname{sTab}_{\frac{1}{2}}(F)$ and $\operatorname{sTab}_{-}(F)=\operatorname{sTab}(F)$. All of the definitions above also make sense for tables in $\operatorname{sTab}_{\frac{1}{2}}(F)$.

\section{Highest Weight THEORY FOR EVEN MULtipliCity NiLPOTENT ELEMENTS}

The aim of this section is to prove Theorem [5.13. One important tool is the algorithm of Barbasch and Vogan to determine the associated variety of the annihilator of a highest weight module in a classical Lie algebra; this is discussed in $\$ 5.2$, and we give a slight modification in Algorithm 5.3. This algorithm is used in conjunction with Corollary 3.13 and Theorem 4.6 to help prove Theorem 5.13,

5.1. Notation. We recap some of the notation given in the introduction and give explicit choices for the notation from 22.1 .

We fix a positive integer $n$ and a sign $\phi \in\{ \pm\}$. Let $V=\mathbb{C}^{2 n}$ be the $2 n$-dimensional vector space with standard basis $\left\{e_{-n}, \ldots, e_{-1}, e_{1}, \ldots, e_{n}\right\}$ and nondegenerate bilinear form $(\cdot, \cdot)$ defined by $\left(e_{i}, e_{j}\right)=0$ if $i$ and $j$ have the same sign, and $\left(e_{i}, e_{-j}\right)=\delta_{i, j},\left(e_{-i}, e_{j}\right)=\phi \delta_{i, j}$ for $i, j \in\{1, \ldots, n\}$. Let $\tilde{G}=G_{2 n}^{\phi}=\left\{x \in \mathrm{GL}_{2 n} \mid\left(x v, x v^{\prime}\right)=\left(v, v^{\prime}\right)\right.$ for all $\left.v, v^{\prime} \in V\right\}$, and $\mathfrak{g}=\mathfrak{g}_{2 n}^{\phi}=\left\{x \in \mathfrak{g l}_{2 n} \mid\left(x v, v^{\prime}\right)=-\left(v, x v^{\prime}\right)\right.$ for all $\left.v, v^{\prime} \in V\right\}$ be the Lie algebra of $\tilde{G}$. So $\tilde{G}=\mathrm{O}_{2 n}$ and $\mathfrak{g}=\mathfrak{s o}_{2 n}$ if $\phi=+$, and $\tilde{G}=\mathrm{Sp}_{2 n}$ and $\mathfrak{g}=\mathfrak{s p}_{2 n}$ if $\phi=-$. We write $G$ for the derived group of $\tilde{G}$, so $G=\tilde{G}$ in the type $\mathrm{C}$ case, and $G=\mathrm{SO}_{2 n}$ in the type D case; note that equivalently $G$ is the identity component of $\tilde{G}$.

Let $\left\{e_{i, j} \mid i, j=-n, \ldots,-1,1, \ldots, n\right\}$ be the standard basis of $\mathfrak{g l}_{2 n}$, and define $f_{i, j}=$ $e_{i, j}-\eta_{i, j} e_{-j,-i}$ where $\eta_{i, j}=1$ if $i$ and $j$ have the same sign and $\eta_{i, j}=\phi$ is $i$ and $j$ have different signs. Then the standard basis of $\mathfrak{g}$ is $\left\{f_{i, j} \mid i+j<0\right\}$ if $\phi=+$ and $\left\{f_{i, j} \mid i+j \leq 0\right\}$ if $\phi=-$. Let $\mathfrak{t}=\left\langle f_{i, i} \mid i=1, \ldots, n\right\rangle$ be the standard Cartan subalgebra of $\mathfrak{g}$ of diagonal matrices. We define $\left\{\epsilon_{i}^{\prime} \mid i=1, \ldots, n\right\}$ to be the basis of $\mathfrak{t}^{*}$ dual to $\left\{f_{i, i} \mid i=1, \ldots, n\right\}$ and let $\epsilon_{i}=-\epsilon_{i}^{\prime}$.

Recall that $W$ denotes the Weyl group of $\mathfrak{g}$ with respect to $\mathfrak{t}$. Let $T$ be the maximal torus of $\tilde{G}$ corresponding to $\mathfrak{t}$, and let $\tilde{W}=N_{\tilde{G}}(T) / T$. Note that in the case $\mathfrak{g}=\mathfrak{s o}_{2 n}, \tilde{W}$ is a Coxeter group of type $\mathrm{C}_{n}$ which contains $W$ as a subgroup of index 2 .

We recall that nilpotent $\tilde{G}$-orbits in $\mathfrak{g}$ are parameterized by partitions $\mathbf{p}$, such that each even (respectively odd) part of $\mathbf{p}$ has even multiplicity when $\mathfrak{g}=\mathfrak{s o}_{2 n}$ (respectively $\mathfrak{s p}_{2 n}$ ). For $\mathfrak{g}=\mathfrak{s o}_{2 n}$, we also recall that a nilpotent $\tilde{G}$-orbit parameterized by $\mathbf{p}$ is a single $G$-orbit unless all parts of $\mathbf{p}$ are even and of even multiplicity. In this latter case, where we say that $\mathbf{p}$ is very even, and the $\tilde{G}$-orbit parameterized by $\mathbf{p}$ splits into two $G$-orbits. 
We recall the structure of the component group $\tilde{C}$ of the centralizer of $e$ in $\tilde{G}$. Suppose $e \in \mathfrak{g}$ lies in the nilpotent $\tilde{G}$-orbit corresponding to the partition p. Then $\tilde{C} \cong \mathbb{Z}_{2}^{d}$, where $d$ is the number of distinct even parts of $\mathbf{p}$ if $\mathfrak{g}=\mathfrak{s p}_{2 n}$ and the number of distinct odd parts of $\mathbf{p}$ if $\mathfrak{g}=\mathfrak{s o}_{2 n}$, see for example [Ja, $\left.\S 3.13\right]$. We write $C$ for the component group of the centralizer of $e$ in $G$. We note that $C$ is equal to $\tilde{C}$ unless $\mathfrak{g}=\mathfrak{s o}_{2 n}$ and $\mathbf{p}$ has an odd part, in which case $C$ has index 2 in $\tilde{C}$.

For the remainder of the paper we fix an even multiplicity partition $\mathbf{p}=\left(p_{1}^{2}, \ldots, p_{r}^{2}\right)$ of $2 n$, where $p_{i} \geq p_{i+1}$ for each $i$. The symmetric pyramid of $\mathbf{p}$ is defined in the introduction, or equivalently it is the symmetric pyramid with row lengths given by $\mathbf{p}$ as defined in $\$ 4.4$. The table with frame the symmetric pyramid of $\mathbf{p}$ and with boxes filled by $-n, \ldots,-1,1, \ldots, n$ from left to right and top to bottom is called the coordinate pyramid associated to $\mathbf{p}$ and denoted by coord $(\mathbf{p})$; an example of a coordinate pyramid is given in the introduction.

More generally we say an s-frame $F$ is associated to $\mathbf{p}$ if $\operatorname{part}(F)=\mathbf{p}$. We define the coordinate table with frame $F$ to be the element $\operatorname{coord}(F) \in \operatorname{sTab}^{\leq}(F)$ with boxes filled by $-n, \ldots,-1,1, \ldots, n$ such that

- we obtain $\operatorname{coord}(F)$ from $\operatorname{coord}(\mathbf{p})$ by rearranging rows and keeping entries in the same boxes, and

- whenever $F$ has 2 rows of the same length, then the entries in the lower row of $\operatorname{coord}(F)$ are greater than those in the higher row.

It is easy to see that these conditions determine coord $(F)$ uniquely. For example

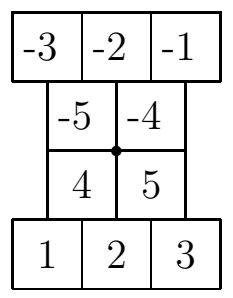

is a coordinate table.

Let $F$ be an s-frame associated to $\mathbf{p}$ and $\operatorname{coord}(F)$ be the coordinate table with frame $F$. In order to define some elements and subalgebras of $\mathfrak{g}$ associated to $\mathbf{p}$, we need to fix an explicit embedding of coord $(F)$ in the plane. To do this we declare that the central point is the origin and the boxes have size $2 \times 2$. Then given $i \in\{ \pm 1, \ldots, \pm n\}$, let $\operatorname{col}(i)$ be the $x$-coordinate of the centre of the box labelled by $i$, however we use row $(i)$ to denote the row containing $i$ as indicated by the labelling of rows from $\$ 4.4$.

We define the nilpotent element $e$ with Jordan type $\mathbf{p}$ by $e=\sum f_{i, j}$, where we sum over all $i, j$ such that $i, j$ are positive and $j$ is in the box immediately to the right of $i$, and define $h=\sum_{i=1}^{n}-\operatorname{col}(i) f_{i, i}$. For the example above we have $e=f_{1,2}+f_{2,3}+f_{4,5}$ and $h=2 f_{1,1}-2 f_{3,3}+f_{4,4}-f_{5,5}$. Now the ad $h$ eigenspace decomposition is given by

$$
\mathfrak{g}(k)=\left\langle f_{i, j} \mid \operatorname{col}(j)-\operatorname{col}(i)=k\right\rangle,
$$

and we can find $f \in \mathfrak{g}(-2)$ such that $(e, h, f)$ is an $\mathfrak{s l}_{2}$-triple. We define the subspaces $\mathfrak{p}, \mathfrak{h}$, $\mathfrak{n}$ and $\mathfrak{k}$ of $\mathfrak{g}$ as in 92.1 , and now we have

$$
\begin{gathered}
\mathfrak{p}=\left\langle f_{i, j} \mid \operatorname{col}(i) \leq \operatorname{col}(j)\right\rangle, \quad \mathfrak{n}=\left\langle f_{i, j} \mid \operatorname{col}(i)>\operatorname{col}(j)\right\rangle, \\
\mathfrak{h}=\left\langle f_{i, j} \mid \operatorname{col}(i)=\operatorname{col}(j)\right\rangle, \quad \mathfrak{k}=\left\langle f_{i, j} \mid \operatorname{col}(i)=\operatorname{col}(j)+1\right\rangle .
\end{gathered}
$$

This gives all the information needed to define the finite $W$-algebra $U(\mathfrak{g}, e)$ as in $\oiint 2.1$. 
We note that $\mathfrak{t}, e$ and $\mathfrak{h}$ are chosen so that $h \in \mathfrak{t}$ and that $\mathfrak{t}^{e}$ is a maximal toral subalgebra of $\mathfrak{g}^{e} ; \mathfrak{t}^{e}$ has a basis given by the elements $\sum_{\operatorname{row}(j)=i} f_{j, j}$ as $i$ ranges over the rows in the lower half of $F$. The Levi subalgebra $\mathfrak{g}_{0}$ is spanned by $\left\{f_{i, j} \mid \operatorname{row}(i)=\operatorname{row}(j)\right\}$. We have $\mathfrak{b}_{0}=\mathfrak{p}_{0}=\mathfrak{p} \cap \mathfrak{g}_{0}$ is a Borel subalgebra of $\mathfrak{g}_{0}$.

The definitions above only depend on $\mathbf{p}$ and not on the choice of frame $F$ associated to p. Next we define the parabolic algebra subalgebra

$$
\mathfrak{q}_{F}=\left\langle f_{i, j} \mid \operatorname{row}(i) \leq \operatorname{row}(j)\right\rangle .
$$

Then $\mathfrak{g}_{0}$ is a Levi subalgebra of $\mathfrak{q}_{F}$, and we define $\mathfrak{b}_{F}$ to be the Borel subalgebra of $\mathfrak{g}$ generated by $\mathfrak{b}_{0}$ and the nilradical of $\mathfrak{q}_{F}$.

In the case $F$ is the symmetric pyramid associated to $\mathbf{p}$, we omit the subscript $F$, so we just write $\mathfrak{q}$ and $\mathfrak{b}$. In particular $\mathfrak{b}=\left\langle f_{i, j} \mid i \leq j\right\rangle$ is the Borel subalgebra consisting of upper triangular matrices in $\mathfrak{g}$.

To $A \in \operatorname{sTab}_{\phi}^{\leq}(F)$ we associate $\lambda_{A} \in \mathfrak{t}^{*}$ by declaring that $\lambda_{A}=\sum_{i=1}^{n} a_{i} \epsilon_{i}$ where $a_{i}$ is the number in the box of $A$ occupying the same position as $-i$ in coord $(F)$. For example, if $A$ is the s-table in (4.12), then $\lambda_{A}=-7 \epsilon_{6}+3 \epsilon_{5}-8 \epsilon_{4}-4 \epsilon_{3}+2 \epsilon_{2}+5 \epsilon_{1}$. The $W_{0}$-orbit of $\lambda_{A}$ is denoted by $\Lambda_{A} \in \mathfrak{t}^{*} / W_{0}$. Thus we can associate to $A$ the highest weight module $L\left(\Lambda_{A}, \mathfrak{q}_{F}\right)$ as defined in 93.3 , we denote $L\left(\Lambda_{A}, \mathfrak{q}_{F}\right)$ by $L(A)$ for short noting that $A$ encodes the parabolic subalgebra $\mathfrak{q}_{F}$. We define $\operatorname{sTab}_{\phi}^{+}(F)$ to be the subset of $\operatorname{sTab}_{\phi}^{\leq}(F)$ consisting of s-tables $A$ such that $L(A)$ is finite dimensional. We write $\operatorname{Pyr}_{\phi}(\mathbf{p}), \operatorname{Pyr}_{\phi}^{+}(\mathbf{p})$, and $\operatorname{Pyr}_{\phi}^{\leq}(\mathbf{p})$, for sTab $\operatorname{Tab}_{\phi}(F)$, $\operatorname{sTab}_{\phi}^{+}(F)$, and $\operatorname{sTab}_{\phi}^{\leq}(F)$ respectively when $F$ is the symmetric pyramid associated to $\mathbf{p}$.

Let $A \in \operatorname{sTab}_{\phi}^{\leq}(F)$. Then the weight $\lambda_{A}$ satisfies the condition $\left\langle\lambda_{A}, \alpha^{\vee}\right\rangle \notin \mathbb{Z}_{>0}$ for all $\alpha \in \Phi_{0}^{+}$, because the rows of $A$ are weakly increasing. The condition that the entries of $A$ either all lie in $\mathbb{Z}$ or all lie in $\mathbb{Z}+\frac{1}{2}$ (the latter only if $\mathfrak{g}$ is of type $\mathrm{D}$ ) implies that $\lambda_{A} \in \mathfrak{t}_{\mathbb{Z}}^{*}$.

5.2. Associated varieties of primitive ideals. In this subsection we recall from [BV] how to calculate the associated variety of a primitive ideal in the universal enveloping algebras of the classical Lie algebras. For our purposes we restrict to $\mathfrak{g}$ of type $\mathrm{C}$ or D. For a primitive ideal $I$ of $U(\mathfrak{g})$ we recall that the associated variety $\mathcal{V} \mathcal{A}(I)$ of $I$ is the closure of a nilpotent $G$-orbit, see for example [Ja, §9].

By Duflo's Theorem (see [Du] ), it suffices to calculate the associated variety of annihilators of irreducible highest weight modules. These modules are defined in terms of the Borel subalgebra $\mathfrak{b}$ and the Cartan subalgebra $\mathfrak{t} \subseteq \mathfrak{b}$ from $\$ 5.1$. In this paper, we only consider integral weights $\lambda \in \mathfrak{t}_{\mathbb{Z}}^{*}$. For such $\lambda$, we write $L(\lambda)$ for the irreducible highest weight $U(\mathfrak{g})$ module with highest weight $\lambda-\rho$ with respect to $\mathfrak{b}$. We recall that we say that $\lambda$ is anti-dominant if $\left\langle\lambda, \alpha^{\vee}\right\rangle \in \mathbb{Z}_{\leq 0}$ for all $\alpha \in \Phi^{+}$. Also we recall that for any $\mu \in \mathfrak{t}_{\mathbb{Z}}^{*}$ there exists $w \in W$ and antidominant $\lambda \in \mathfrak{t}_{\mathbb{Z}}^{*}$ such that $\mu=w \lambda$.

A weight $\lambda \in \mathfrak{t}^{*}$ is regular if $\left\langle\lambda, \alpha^{\vee}\right\rangle \neq 0$ for all $\alpha \in \Phi$. For any regular, anti-dominant weights $\lambda_{1}$ and $\lambda_{2}$, and $w \in W$ it is well known that

$$
\mathcal{V} \mathcal{A}\left(\operatorname{Ann} L\left(w \lambda_{1}\right)\right)=\mathcal{V} \mathcal{A}\left(\operatorname{Ann} L\left(w \lambda_{2}\right)\right),
$$

see for example [Ja, $\S 9.12]$. Recall that $\tilde{W}=N_{\tilde{G}}(T) / T$, so when $\mathfrak{g}$ is of type D it contains $W$ as a subgroup of index 2. Suppose $\mathfrak{g}$ is of type $\mathrm{D}$ and let $s \in \tilde{W}$ be the element that fixes all $\epsilon_{i}$ except for $\epsilon_{1}$, which it sends to $-\epsilon_{1}$, so $s \notin W$. If $\lambda \in \mathfrak{t}_{\mathbb{Z}}^{*}$ is antidominant, then it 
is easy to check that $s \lambda$ is too. It follows that $\mathcal{V} \mathcal{A}(\operatorname{Ann} L(w \lambda))=\mathcal{V} \mathcal{A}(\operatorname{Ann} L(w s \lambda))$ for any $w \in W$, so (5.2) holds for all $w \in \tilde{W}$.

The following is an algorithm adapted from [BV] to determine the associated variety of a primitive ideal in types $\mathrm{C}$ and $\mathrm{D}$.

Algorithm 5.3. Input: $\lambda=\sum a_{i} \epsilon_{i} \in \mathfrak{t}_{\mathbb{Z}}^{*}$.

Step 1: Let $\mathbf{q}=\operatorname{RS}\left(a_{n}, \ldots, a_{1},-a_{1}, \ldots,-a_{n}\right)$. In type $\mathrm{D}$ when calculating the RobinsonSchensted algorithm, if zeros occur, then we treat the two zeros closest to the middle of $\left(a_{n}, \ldots, a_{1},-a_{1}, \ldots,-a_{n}\right)$ as if the first zero is larger than the second.

Step 2: Put $\mathbf{q}=\left(q_{1} \leq q_{2} \leq \cdots \leq q_{m}\right)$ into ascending order. By inserting zero into $\mathbf{q}$ if necessary, in type $\mathrm{C}$ assume that $\mathbf{q}$ has an odd number of parts, and in type $\mathrm{D}$ assume that $\mathbf{q}$ has an even number of parts. For $i=1 \ldots m$ let $r_{i}=q_{i}+i-1$ to create the list $\left(r_{i}\right)$. Let $\left(2 s_{1}, \ldots, 2 s_{l}\right)$ be the sublist of $\left(r_{i}\right)$ consisting of even numbers, and let $\left(2 t_{1}+1, \ldots, 2 t_{k}+1\right)$ be the sublist of $\left(r_{i}\right)$ consisting of odd numbers.

Step 3: Let $\left(u_{i}\right)$ be list obtained by sorting the concatenation of $\left(s_{i}\right)$ with $\left(t_{i}\right)$. Now let $\left(s_{i}^{\prime}\right)$ and $\left(t_{i}^{\prime}\right)$ be the sublists of $\left(u_{i}\right)$ consisting of the terms with odd and even indices, respectively. Let $\left(r_{i}^{\prime}\right)$ denote the list obtained by sorting the concatenation of $\left(2 s_{i}^{\prime}\right)$ and $\left(2 t_{i}^{\prime}+1\right)$ in type $\mathrm{C}$, and $\left(2 s_{i}^{\prime}+1\right)$ and $\left(2 t_{i}^{\prime}\right)$ in type D. Finally let $q_{i}^{\prime}=r_{i}^{\prime}+1-i$ to form the partition $\mathbf{q}^{\prime}$.

The following corollary is a consequence of [BV, Theorem 18].

Corollary 5.4. Let $\lambda \in \mathfrak{t}_{\mathbb{Z}}^{*}$ and let $\mathbf{q}^{\prime}$ be the output of Algorithm 5.3.

(i) Suppose $\mathfrak{g}$ is of type $C$ or $\mathfrak{g}$ is of type $D$ and $\mathbf{p}^{\prime}$ is not very even. Then $\mathcal{V} \mathcal{A}(\operatorname{Ann} L(\lambda))$ is equal to the closure of the nilpotent $\tilde{G}$-orbit corresponding to the partition $\mathbf{q}^{\prime}$.

(ii) Suppose $\mathfrak{g}$ is of type $D$ and $\mathbf{p}^{\prime}$ is very even. Then $\mathcal{V} \mathcal{A}(\operatorname{Ann} L(\lambda))$ is equal to the closure of one of the two $G$-orbits in the nilpotent $\tilde{G}$-orbit corresponding to the partition $\mathbf{q}^{\prime}$.

Proof. To prove Corollary 5.4 we present the algorithm given in [BV]. This is broken in to four steps. After each step we make some remarks and explain and justify some adaptations that we make to get Algorithm [5.3,

Input: The algorithm from [BV] takes as input an element $w \in \bar{W}$ and calculates the associated variety of the annihilator of $L(w \lambda)$ where $\lambda \in \mathfrak{t}_{\mathbb{Z}}^{*}$ is antidominant and regular. Let $\sigma=n \epsilon_{n}+(n-1) \epsilon_{n-1}+\cdots+\epsilon_{1} \in \mathfrak{t}^{*}$, and identify $w$ with $w \sigma$. Then identify $w \sigma=\sum_{i=1}^{n} a_{i} \epsilon_{i}$ with the list $\left(a_{n}, \ldots, a_{1},-a_{1}, \ldots,-a_{n}\right)$.

Adaptation: Instead in Algorithm 5.3 we take as input $\mu=w \lambda=\sum_{i=1}^{n} b_{i} \epsilon_{i}$ and we identify $\mu$ with the list $\left(b_{n}, \ldots, b_{1},-b_{1}, \ldots,-b_{n}\right)$. This is justified below.

Step 1: Calculate part $\left(\operatorname{RS}\left(a_{n}, \ldots, a_{1},-a_{1}, \ldots, a_{n}\right)\right)$, and let $\mathbf{q}$ be the transpose partition.

Remarks and adaptation: In [BV] Barbasch and Vogan use a "dual" version of the RobinsonSchensted algorithm which results in the transpose partition to that obtained from the version given in \$4.2, so they do not need to take the transpose here.

It follows from Lemma 4.4 that $\operatorname{part}\left(\operatorname{RS}\left(a_{n}, \ldots, a_{1},-a_{1}, \ldots,-a_{n}\right)\right)$ is the transpose of $\operatorname{part}\left(\operatorname{RS}\left(-a_{n}, \ldots,-a_{1}, a_{1}, \ldots, a_{n}\right)\right)$. Therefore, we obtain $\mathbf{q}$ by applying the RobinsonSchensted algorithm to $\left(-a_{n}, \ldots,-a_{1}, a_{1}, \ldots, a_{n}\right)$.

Note that $-\sigma$ is antidominant, and $w(-\sigma)=-\sigma w=\sum_{i=1}^{n}-a_{i} \epsilon_{i}$. This means that for $i, j=1, \ldots, n$ we have $-a_{i}<-a_{j}$ precisely when $b_{i}<b_{j}$ and $-a_{i}<a_{j}$ precisely when $b_{i}<-b_{j}$. This implies that

$$
\operatorname{part}\left(\operatorname{RS}\left(-a_{n}, \ldots,-a_{1}, a_{1}, \ldots, a_{n}\right)\right)=\operatorname{part}\left(\operatorname{RS}\left(b_{n}, \ldots, b_{1},-b_{1}, \ldots,-b_{n}\right)\right) .
$$


So in Algorithm 5.3 we instead calculate $\mathbf{q}=\operatorname{part}\left(\operatorname{RS}\left(b_{n}, \ldots, b_{1},-b_{1}, \ldots,-b_{n}\right)\right)$.

Step 2: Put $\mathbf{q}$ into ascending order; say $\mathbf{q}=\left(q_{1} \leq q_{2} \leq \cdots \leq q_{2 k+1}\right)$ by inserting a zero if necessary to ensure there are an odd number of parts. For $i=1 \ldots 2 k+1$, let $r_{i}=q_{i}+i-1$ to create the list $\left(r_{i}\right)$. Let $\left(2 s_{1}, \ldots, 2 s_{k+1}\right)$ be the sublist of $\left(r_{i}\right)$ consisting of even numbers, and let $\left(2 t_{1}+1, \ldots, 2 t_{k}+1\right)$ be the sublist of $\left(r_{i}\right)$ consisting of odd numbers.

Step 2a: Only do the following in the type D case. If $s_{1} \neq 0$, then replace the list $\left(t_{1}, \ldots, t_{k}\right)$ with the list $\left(0, t_{1}+1, \ldots, t_{k}+1\right)$. If $s_{1}=0$, then replace the list $\left(s_{1}, \ldots, s_{k+1}\right)$ with the list $\left(s_{2}-1, \ldots, s_{k+1}-1\right)$.

Remarks and adaptation: The fact that the list $\left(s_{i}\right)$ has $k+1$ elements and the list $\left(t_{i}\right)$ has $k$ elements (before the change in the type D case) is due to [BV, Proposition 17].

In the type $\mathrm{D}$ case, suppose instead that we modify $\mathbf{q}$ by possibly adding a zero to the start to ensure that it has an even number entries. Then we can define the lists $\left(s_{i}\right)$ and $\left(t_{i}\right)$ using the same procedure. One can check that these lists are exactly the same as those obtained by assuming that $\mathbf{q}$ has an odd number of entries and then doing Step 2a. Therefore, we remove this step in Algorithm 5.3.

Step 3: Do this step exactly as Step 3 in Algorithm 5.3.

Output: The nilpotent $\tilde{G}$-orbit corresponding to the partition $\mathbf{q}^{\prime}$.

By [BV, Theorem 18] the partition $\mathbf{q}^{\prime}$ corresponds to a nilpotent $\tilde{G}$-orbit, and when $\mathfrak{g}$ is of type $\mathrm{C}$, then $\mathcal{V} \mathcal{A}(\operatorname{Ann} L(w \lambda))$ is precisely this orbit. In the case $\mathfrak{g}$ is of type $\mathrm{D}$ and $\mathbf{q}^{\prime}$ is not very even, then [BV, Theorem 18] gives that $\mathcal{V} \mathcal{A}(\operatorname{Ann} L(w \lambda))$ is the closure of the nilpotent $\mathrm{SO}_{2 n}$-orbit corresponding to $\mathbf{q}^{\prime}$. If $\mathbf{q}^{\prime}$ is very even, then [BV, Theorem 18] gives that the associated variety is the closure of one of the $\mathrm{SO}_{2 n}$-orbits contained in the $\tilde{G}$-orbit corresponding to $\mathbf{q}^{\prime}$. We do not need to know which orbit for our purposes.

Our convention about zeros in the type D case from Algorithm 5.3 is due to [BV, p. 173].

So far we have only justified that Algorithm 5.3 works the case that $\lambda$ is regular, however we can remove this assumption using [Jo2, Lemma 2.4].

We recall that the array $\left(\begin{array}{l}s \\ t\end{array}\right)$ obtained by placing the list $\left(s_{i}\right)$ on the top row and the list $\left(t_{i}\right)$ is called a symbol associated to q. This notation was introduced by Lusztig in [Lu]. We call the concatenated list $\left(u_{i}\right)$ the content of the symbol $\left(\begin{array}{l}s \\ t\end{array}\right)$, or simply the content of $\mathbf{q}$.

5.3. The component group action. At present the only cases where the nontrivial action of $\tilde{C}$ on the set of isomorphism classes of finite dimensional irreducible $U(\mathfrak{g}, e)$-modules is known is where $\mathfrak{g}=\mathfrak{s p}_{2 n}$ or $\mathfrak{g}=\mathfrak{s o}_{2 n}$ and the Jordan type of $e$ has an even number of Jordan blocks all of the same size, i.e. the case $\mathbf{p}=\left(l^{2 r}\right)$, so that the symmetric pyramid of $\mathbf{p}$ is a rectangle. The description of the action depends on the notion of the $\sharp$-element of a list of complex numbers.

Given a list $\left(a_{1}, \ldots, a_{2 k+1}\right)$ of complex numbers let $\left\{\left(a_{1}^{(i)}, \ldots, a_{2 k+1}^{(i)}\right) \mid i \in I\right\}$ be the set of all permutations of this list which satisfy $a_{2 j-1}^{(i)}+a_{2 j}^{(i)} \in \mathbb{Z}_{>0}$ for each $j=1, \ldots, k$. Assuming that such rearrangements exist, we define the $\sharp$-element of $\left(a_{1}, \ldots, a_{2 k+1}\right)$ to be the unique maximal element of the set $\left\{a_{2 k+1}^{(i)} \mid i \in I\right\}$. On the other hand, if no such rearrangements exist, we say that the $\sharp$-element of $\left(a_{1}, \ldots, a_{2 k+1}\right)$ is undefined. For example, the $\sharp$-element of $(-3,-1,2)$ is -3 , whereas the $\sharp$-element of $(-3,-2,1)$ is undefined.

We abuse notation somewhat about saying that the $\sharp$-element of a list of numbers with an even number of elements is the $\sharp$-element of that list with zero inserted.

The following lemma is easy to prove and is required in the proof of Theorem 5.13. 
Lemma 5.5. If $\left(a_{1}, \ldots, a_{2 k}\right)$ is a list of integers which satisfies $a_{2 i-i}+a_{2 i}>0$ for $i=1, \ldots, k$, and $b_{1}, \ldots, b_{2 k}$ is same list sorted into weakly increasing order, then $b_{i}+b_{2 k+1-i}>0$ for $i=1, \ldots, 2 k$.

For the next few paragraphs let $F$ denote the unique s-frame which satisfies part $(F)=$ $\left(l^{2 r}\right)$, where $r$ and $l$ are fixed positive integers, and $l$ is even if $\mathfrak{g}=\mathfrak{s p}_{2 n}$ and $l$ is odd if $\mathfrak{g}=\mathfrak{s o}_{2 n}$. In this case we have $\tilde{C} \cong \mathbb{Z}_{2}=\langle c\rangle$ and we define an operation of $c$ on $\operatorname{sTab}^{+}(F) \subseteq \operatorname{sTab}^{\leq}(F)$ as follows. Let $A \in \mathrm{sTab}^{+}(F)$ and let $a_{1}, \ldots, a_{l}$ be row -1 of $A$. By [Bro2, Theorem 1.2] the $\sharp$-element of $a_{1}, \ldots, a_{l}$ is defined; let $a$ be this number. We declare that $c \cdot A \in \operatorname{sTab}^{\leq}(F)$ is the s-table with the same rows as $A$, except with one occurrence of $a$ replaced with $-a$ in row -1 , and one occurrence of $-a$ replaced with $a$ in row 1. Then [Bro2, Theorem 1.3] says that $c \cdot L(A)=L(c \cdot A)$. An example of this action is

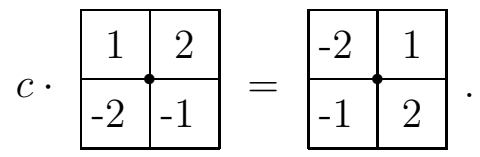

The next lemma helps explain what happens when the Robinson-Schensted algorithm is applied to $A$ in the case that $r=2$ and the $\sharp$-element of row -1 of $A$ is defined; it is required in the proof of Theorem 5.13. For this lemma, in the case that $l$ is odd, we use the zero convention for calculating the Robinson-Schensted algorithm from Algorithm 5.3.

Lemma 5.6. Let $A$ be a rectangular s-frame with distinct entries and with $\operatorname{part}(A)=\left(l^{2}\right)$. Then the $\sharp$-element of row -1 of $A$ is defined if and only if $\operatorname{RS}(A)=(l, l)$ or $\operatorname{RS}(A)=$ $(l+1, l-1)$. More specifically, let a be the $\sharp$-element of row -1 of $A$. If $a \geq 0$, then $\operatorname{RS}(A)=(l, l)$. If $a<0$, then $\operatorname{RS}(A)=(l+1, l-1)$.

Proof. Let $a_{1}, \ldots, a_{l}$ be row -1 of $A$, and suppose that the $\sharp$-element of $\left(a_{1}, \ldots, a_{l}\right)$ is defined and is $a_{s}$. Suppose that $a_{s} \geq 0$. If $a_{s}>0$ or if $l$ is even, then by Lemma 5.5 we have that $a_{i}+a_{l+1-i}>0$ for $i=1, \ldots, l$. Thus for $i=1, \ldots, l$ we have that $a_{i}>-a_{l+1-i}$, which implies that $\operatorname{RS}(A)=(l, l)$. If $a_{s}=0$ and $l$ is odd, then we must have that $s=(l+1) / 2$, and we have that $a_{i}+a_{l+1-i}>0$ for $i=1, \ldots,(l-1) / 2$. Thus for $i=1, \ldots,(l-1) / 2,(l+3) / 2, \ldots, l$, we have that $a_{i}>-a_{l+1-i}$. Since we also count $0=a_{s}$ as greater than $0=-a_{s}$ when calculating the Robinson-Schensted algorithm, we have that $\operatorname{RS}(A)=(l, l)$. Now suppose that $a_{s}<0$. Then $1 \leq s \leq(l+1) / 2$. Since $a_{s}$ is the sharp element, $a_{1}, \ldots, a_{s},-a_{l+1-s}, \ldots,-a_{1}$ is an increasing sequence of length $l+1$ in word $(A)$. Combining this with the fact that the rows of $A$ are increasing and using Lemma 4.3, we see that $\operatorname{RS}(A) \geq(l+1, l-1)$. Also by Lemma 5.5 we must have that

- $a_{j}+a_{l+1-j}>0$ for $1 \leq j<s$,

- $a_{j}+a_{l+2-j}>0$ for $s<j \leq(l+1) / 2$, and

- $a_{l / 2+1}>0$ if $l$ is even.

The only way that $\operatorname{RS}(A)$ could be larger than $(l+1, l-1)$ in the dominance order is if the first term is larger than $l+1$. By Lemma 4.3, this is only possible if $\operatorname{word}(A)$ contains a weakly increasing subsequence of length at least $l+2$, which can only happen if there exists $j$ such that $a_{j}<-a_{l+2-j}$, which cannot happen due to the above conditions.

Now suppose that $\operatorname{part}(\operatorname{RS}(A))=(l, l)$. Then we must have that $a_{i}+a_{l+1-i}>0$ for all $i$ such that $1 \leq i<(l+1) / 2$, so the $\sharp$-element is defined.

Finally suppose that part $(\operatorname{RS}(A))=(l+1, l-1)$. So for some $j$ we have that 


$$
\operatorname{RS}(A)=\begin{array}{|c|c|c|c|c|c|c|c|c|c|}
\hline a_{1} & a_{2} & \ldots & a_{j-1} & a_{j+1} & a_{j+2} & \ldots & a_{l} & \multicolumn{2}{|c|}{} \\
\hline-a_{l} & -a_{l-1} & \ldots & -a_{l-j+2} & a_{j} & -a_{l-j+1} & \ldots & -a_{3} & -a_{2} & -a_{1} \\
\hline
\end{array}
$$

This implies that $j \leq l / 2$, otherwise we would have $-a_{j}<a_{l-j+1}$ and $-a_{j}<a_{j}<-a_{l-j+1}$, which is a contradiction. Also (5.7) tells us that the following sums are all positive integers: $a_{1}+a_{l}, a_{2}+a_{l-1}, \ldots, a_{j-1}+a_{l-j+2}$. We also have that $a_{j+1}+a_{l-j+1}, \ldots, a_{l}+a_{2}$ are all positive, because during the Robinson-Schensted algorithm $-a_{l-j+1}$ must bump $a_{j+1},-a_{l-j}$ must bump $a_{j+2}$, and so on. Thus the $\sharp$-element of row -1 of $A$ is defined.

Remark 5.8. It is useful in the proof of Theorem 5.13 to consider explicitly calculating $\operatorname{RS}(c \cdot A)$ when $A$ has 2 rows, the $\sharp$-element of $A$ is positive, and all the elements of row -1 of $A$ are distinct. Let $a_{1}, \ldots, a_{l}$ be row -1 of $A$, and let $a_{s}$ be the $\sharp$-element of $a_{1}, \ldots, a_{l}$. So we must have that $s>l / 2$. If $s>(l+1) / 2$, then by calculating the Robinson-Schensted algorithm on both words, observe that the word $a_{1}, \ldots, a_{l},-a_{l}, \ldots,-a_{1}$ is Knuth-equivalent to

$$
\begin{aligned}
& a_{1}, \ldots, a_{l-s},-a_{l}, \ldots,-a_{s+1}, a_{l-s+1},-a_{s}, a_{l-s+2}, \ldots, a_{s-1}, \\
& \quad-a_{s-1}, \ldots,-a_{l-s+2}, a_{s},-a_{l-s+1}, a_{s+1}, \ldots, a_{l},-a_{l-s}, \ldots,-a_{1} .
\end{aligned}
$$

By swapping $a_{l-s+1}$ with $-a_{s}$, and $a_{s}$ with $-a_{l-s+1}$ we get the word

$$
\begin{aligned}
& a_{1}, \ldots, a_{l-s},-a_{l}, \ldots,-a_{s}, a_{l-s+1}, \ldots, a_{s-1}, \\
& \quad-a_{s-1}, \ldots,-a_{l-s+1}, a_{s}, \ldots, a_{l},-a_{l-s}, \ldots,-a_{1} .
\end{aligned}
$$

This in turn is Knuth-equivalent to the word

$$
\begin{aligned}
& a_{1}, \ldots, a_{l-s},-a_{s}, a_{l-s+1}, \ldots, a_{s-1}, a_{s+1}, \ldots, a_{l}, \\
& \quad-a_{l}, \ldots,-a_{s+1},-a_{s-1}, \ldots,-a_{l-s+1}, a_{s},-a_{l-s}, \ldots,-a_{1},
\end{aligned}
$$

which is $\operatorname{word}(c \cdot A)$.

If $s=(l+1) / 2$, then by calculating Robinson-Schensted on both words, observe that the word $a_{1}, \ldots, a_{l},-a_{l}, \ldots,-a_{1}$ is Knuth-equivalent to

$$
a_{1}, \ldots, a_{s-1},-a_{l}, \ldots,-a_{s+1}, a_{s},-a_{s}, a_{s+1}, \ldots, a_{l},-a_{s-1}, \ldots,-a_{1} .
$$

By swapping $a_{s}$ with $-a_{s}$ we get the word

$$
a_{1}, \ldots, a_{s-1},-a_{l}, \ldots,-a_{s}, a_{s}, \ldots, a_{l},-a_{s-1}, \ldots,-a_{1} .
$$

This in turn is Knuth-equivalent to the word

$$
a_{1}, \ldots, a_{s-1},-a_{s}, a_{s+1}, \ldots, a_{l},-a_{l}, \ldots,-a_{s+1}, a_{s},-a_{s-1}, \ldots,-a_{1},
$$

which is $\operatorname{word}(c \cdot A)$.

Now we are in a position to describe an operation of the elements of $\tilde{C}$ on $\operatorname{Pyr}_{\phi}^{+}(\mathbf{p})$ for an arbitrary even multiplicity partition $\mathbf{p}$.

Remark 5.9. In this work we do not verify that the operation of elements of $\tilde{C}$ on $\operatorname{Pyr}_{\phi}^{+}(\mathbf{p})$ defines a group action of $\tilde{C}$, however this is the case. While we have a proof of this, it is rather lengthy. Moreover, in the future work BroG we will show that the operation of elements of $\tilde{C}$ on $\operatorname{Pyr}_{\phi}^{+}(\mathbf{p})$ corresponds to the action of $\tilde{C}$ on finite dimensional irreducible $U(\mathfrak{g}, e)$-modules. This will imply that we do have a well defined $\tilde{C}$-action on $\operatorname{Pyr}_{\phi}^{+}(\mathbf{p})$. With 
the exception of Corollary 5.18, all of our results holds without knowing that the operation of elements of $\tilde{C}$ on $\operatorname{Pyr}_{\phi}^{+}(\mathbf{p})$ defines a group action.

Let $i_{1}, \ldots, i_{d}$ be such that $i_{j}<i_{j+1}$ and $p_{i_{1}}, \ldots, p_{i_{d}}$ are the minimal the distinct parts of $\mathbf{p}=\left(p_{1}^{2} \geq p_{2}^{2} \geq \cdots \geq p_{r}^{2}\right)$ that are odd (respectively even) when $\mathfrak{g}=\mathfrak{s o}_{2 n}$ ( respectively $\left.\mathfrak{s p}_{2 n}\right)$. By minimal we mean that if $p_{k}=p_{i_{j}}$, then $k \geq i_{j}$. Then we can choose generators $c_{1}, \ldots, c_{d}$ for $\tilde{C} \cong \mathbb{Z}_{2}^{d}$ corresponding to $p_{i_{1}}, \ldots, p_{i_{d}}$. More specifically, in type $\mathrm{C}$ we set

$$
c_{k}=\sum_{\begin{array}{c}
-n \leq i, j \leq n \\
\operatorname{col}(i)=\operatorname{col}(j) \\
\operatorname{row}(i)=i_{k} \\
\operatorname{row}(j)=-i_{k}
\end{array}} \operatorname{sgn}(\operatorname{col}(i))\left(e_{i, j}+e_{j, i}\right)+\sum_{\begin{array}{r}
-n \leq i \leq n \\
\operatorname{row}(i) \neq \pm i_{k}
\end{array}} e_{i, i},
$$

and in type D we set

$$
c_{k}=\sum_{\begin{array}{c}
-n \leq i, j \leq n \\
\operatorname{col}(i)=\operatorname{col}(j) \\
\operatorname{row}(i)=i_{k} \\
\operatorname{row}(j)=-i_{k}
\end{array}}(-1)^{\operatorname{col}(i) / 2}\left(e_{i, j}+e_{j, i}\right)+\sum_{\begin{array}{c}
-n \leq i \leq n \\
\operatorname{row}(i) \neq \pm i_{k}
\end{array}} e_{i, i} .
$$

Now one can calculate that $c_{k} \in \tilde{H}^{e}$. Furthermore the argument used in [Bro2, Section 6] can be adapted to show that $\tilde{C}$ is generated by $c_{1}, \ldots, c_{d}$. Note that in the type $\mathrm{D}$ case, then any word $w$ in $c_{1}, \ldots, c_{d}$ of even length lies in $C$.

Next we explain how to extend the operation of $c$ on rectangular s-tables given above to any s-table $A \in \operatorname{sTab}_{\phi}^{+}(F)$ as it only involves the middle two rows. We assume the middle two rows of $A$ have odd length if $\mathfrak{g}=\mathfrak{s o}_{2 n}$ and even length if $\mathfrak{g}=\mathfrak{s p}_{2 n}$. We consider the Levi subalgebra $\mathfrak{g}^{\mathfrak{r}}$, where

$$
\mathfrak{r}=\left\langle\sum_{\substack{1 \leq i \leq n \\ \operatorname{row}(i) \neq 1}} f_{i, i}\right\rangle .
$$

Then $\mathfrak{g}^{\mathfrak{r}} \cong \mathfrak{g l}_{n-2 l} \oplus \mathfrak{g}_{2 l}^{\phi}$, where $l$ is the length of row 1 of $F$. Thus by Corollary $3.14, L^{\mathfrak{r}}\left(\Lambda_{A}, \mathfrak{q}_{F}\right)$ is finite dimensional. In turn this implies that the irreducible highest weight module $L\left(A_{1}^{-1}\right)$ for $U\left(\mathfrak{g}_{2 l}^{\phi}, e^{\prime}\right)$, where $e^{\prime} \in \mathfrak{g}_{2 l}^{\phi}$ is a nilpotent element with Jordan type $\left(l^{2}\right)$, is finite dimensional. This means that $c \cdot A_{1}^{-1}$ is defined, so we can define $c \cdot A$ to be the s-table obtained from $A$ by replacing the middle two rows with $c \cdot A_{1}^{-1}$. By Lemma 3.15 and [Bro2, Theorem 6.1] we have that

$$
L(c \cdot A) \cong c \cdot L(A) .
$$

So in particular, $L(c \cdot A)$ is finite dimensional, so $c \cdot A \in \operatorname{sTab}_{\phi}^{+}(F)$.

To define the operation $c_{k}$ on $\operatorname{Pyr}_{\phi}^{+}(F)$ we require the operators $\bar{s}_{i}$ defined in $\$ 4.4$. Another important Levi subalgebra is $\mathfrak{g}^{\mathfrak{s}}$, where

$$
\mathfrak{s}=\left\langle\sum_{i=1}^{n} f_{i, i}\right\rangle .
$$

Then $\mathfrak{g}^{\mathfrak{s}} \cong \mathfrak{g l}_{n}$. The next lemma is required to ensure the operation of $c_{k}$ is defined.

Lemma 5.11. Let $F$ be an s-frame associated to $\mathbf{p}$ and let $A \in \operatorname{sTab}_{\phi}^{+}(F)$. Then

(i) $\operatorname{part}\left(\mathrm{RS}\left(A^{+}\right)\right)=\operatorname{part}\left(A^{+}\right)$and 
(ii) $w \cdot A$ is defined for all words $w$ in $\bar{s}_{1}, \ldots, \bar{s}_{r-1}$.

Proof. By Corollary 3.14 we have that $L^{\mathfrak{s}}\left(\Lambda_{A}, \mathfrak{q}_{F}\right)$ is finite dimensional. This, along with Corollary 3.13 and [Jo1, Corollary 3.3], implies that part $\left(\operatorname{RS}\left(A^{+}\right)\right)=\operatorname{part}\left(A^{+}\right)$giving (i). Now (ii) follows from Lemmas 4.8 and 4.10.

Now we define the action of $c_{k}$ on $A \in \operatorname{Pyr}_{\phi}^{+}(\mathbf{p})$. First we apply $\bar{s}_{i_{k}-1}, \ldots, \bar{s}_{1}$ to $A$ moving row $i_{k}$ in to the middle. Each of these operations is defined by Lemma 5.11. Next we apply $c$ to obtain $A^{\prime}$. Using (5.10), we have that $A^{\prime} \in \operatorname{sTab}_{\phi}^{+}(F)$, where $F$ is the frame obtained from the symmetric pyramid by applying $\bar{s}_{i_{k}-1}, \ldots, \bar{s}_{1}$. We finish by applying the operators $\bar{s}_{1}, \ldots, \bar{s}_{i_{k}-1}$ so that we end up with an element of $\operatorname{Pyr}_{\phi}^{\leq}(\mathbf{p})$. Again by Lemma 5.11 each of these operators is defined. Putting this together we define

$$
c_{k} \cdot A=\bar{s}_{i_{k}} \bar{s}_{i_{k}-1} \ldots \bar{s}_{1} c \bar{s}_{1} \ldots \bar{s}_{i_{k}} \cdot A .
$$

Lemma 5.12. Let $A \in \operatorname{Pyr}_{\phi}^{+}(\mathbf{p})$ (so $L(A)$ is finite dimensional), and let $w$ be a word in $c_{1}, \ldots, c_{d}$. Then $L(w \cdot A)$ is finite dimensional.

Proof. This follows immediately from Lemma 4.8, Corollary 5.4 and (5.10).

Although we do not check here if the operations of the $c_{k}$ on $\operatorname{Pyr}_{\phi}^{+}(\mathbf{p})$ lead to an action of $\tilde{C}$ on $\operatorname{Pyr}_{\phi}^{+}(\mathbf{p})$, we allow ourselves to say that $A, B \in \operatorname{Pyr}_{\phi}^{+}(\mathbf{p})$ are $\tilde{C}$-conjugate if there is a word $w$ in $c_{1}, \ldots, c_{d}$ such that $B=w \cdot A$.

To see some examples of applications of the operator $c_{k}$, if

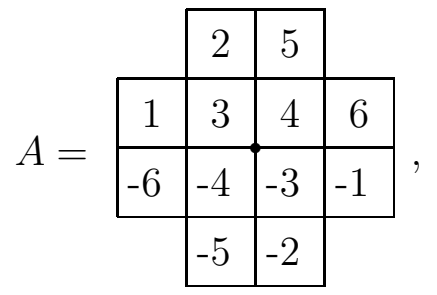

then

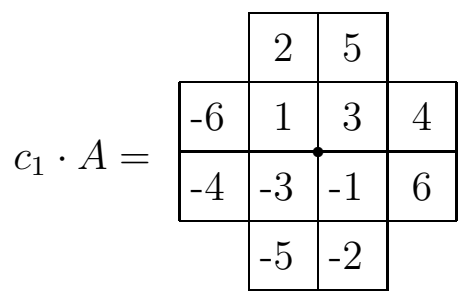

and by conferring with (4.14) we see that

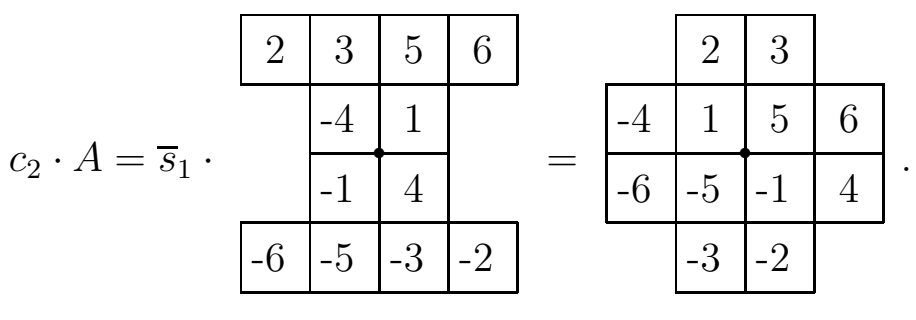




\subsection{Proof of the classification. We define}

$$
\operatorname{Pyr}_{\phi}^{\mathrm{c}}(\mathbf{p})=\left\{A \in \operatorname{Pyr}_{\phi}^{\leq} \mid A \text { is justified row equivalent to column strict }\right\},
$$

however in type $\mathrm{D}$ we used a slightly modified definition of justified row equivalent to column strict. In type $\mathrm{D}$ we say an s-frame $A$ is justified row equivalent to column strict if it is justified row equivalent to column strict in the previous sense, or if the row equivalence class of the left justification of $A$ contains an element $B$ which is column strict everywhere, except the middle 2 boxes of one column of $B$ contain 0 .

Theorem 5.13. Let $\mathfrak{g}=\mathfrak{s p}_{2 n}$ or $\mathfrak{g}=\mathfrak{s o}_{2 n}$. Let $\mathbf{p}$ be an even multiplicity partition of 2 , let $e \in \mathfrak{g}$ be the nilpotent element defined from the symmetric pyramid of $\mathbf{p}$, and let $A \in \mathrm{Pyr}_{\phi}^{\leq}(\mathbf{p})$. Then the $U(\mathfrak{g}, e)$-module $L(A)$ is finite dimensional if and only if $A$ is $\tilde{C}$-conjugate an element of $\operatorname{Pyr}_{\phi}^{\mathrm{c}}(\mathbf{p})$.

Proof. We associate $\lambda_{A}$ to $A$ as in $\$ 5.1$. First observe that [Jo2, Lemma 2.4] reduces the case that $\lambda_{A}$ is non-regular to the case that $\lambda_{A}$ is regular, so we assume that $\lambda_{A}$ is regular. Let $\mathbf{p}=\left(p_{1}^{2}, p_{2}^{2}, \ldots, p_{r}^{2}\right)$, where $p_{i} \geq p_{i+1}$ for all $i$. Let $p_{i_{1}}, \ldots, p_{i_{d}}$ be the minimal distinct parts of $\mathbf{p}$ that are odd (respectively even) when $\mathfrak{g}=\mathfrak{s o}_{2 n}$ (respectively $\mathfrak{s p}_{2 n}$ ). We write $c_{k}$ for the component group action corresponding to $p_{i_{k}}$.

Suppose $A$ is justified row equivalent to column strict. In all cases except the type $\mathrm{D}$ case where $\mathbf{p}$ is very even, $L(A)$ is finite dimensional by Theorem 4.6 and Corollaries 3.13 and 5.4. In the type $\mathrm{D}$ case where $\mathbf{p}$ is very even, by [Lo1, Theorem 1.2.2 (v)] we have that $\overline{G . e} \subseteq \mathcal{V} \mathcal{A}\left(\right.$ Ann $\left.L\left(\lambda_{A}\right)\right)$. Also Theorem 4.6 and Corollary 5.4 give that $\mathcal{V} \mathcal{A}\left(\right.$ Ann $\left.L\left(\lambda_{A}\right)\right)$ is the closure of one of the two nilpotent $G$-orbits corresponding to the partition $\mathbf{p}$, hence we have that $\overline{G . e}=\mathcal{V} \mathcal{A}\left(\operatorname{Ann} L\left(\lambda_{A}\right)\right)$, so by Corollary 3.13 we have that $L(A)$ is finite dimensional. So in all cases for any $w \in \tilde{C}$ we have that $L(w \cdot A)$ is finite dimensional by Lemma 5.12 .

To prove the converse we assume that $L(A)$ is finite dimensional. Let $2 r$ be the number of rows in $A$. We proceed by induction on $r$. In the case that $r=1$, suppose the $A$ has row length $l$. Then by using Corollary 5.4 one checks that $L(A)$ is finite dimensional if and only if $\operatorname{part}(\operatorname{RS}(A))=(l, l)$, or $\operatorname{part}(\operatorname{RS}(A))=(l+1, l-1)$ and $l$ is even and $\mathfrak{g}=\mathfrak{s p}_{2 n}$, or $\operatorname{part}(\operatorname{RS}(A))=(l+1, l-1)$ and $l$ is odd and $\mathfrak{g}=\mathfrak{s o}_{2 n}$. In the former case there is nothing to prove: $A$ is justified row equivalent to column strict by Theorem 4.6. In both of the other cases by Lemma 5.6 we have that $\operatorname{part}\left(\operatorname{RS}\left(c_{1} \cdot A\right)\right)=(l, l)$, so again by Theorem 4.6 $c_{1} \cdot A$ is justified row equivalent to column strict.

Now suppose $r>1$ and let $\mathfrak{r}=\left\langle\sum_{i=n-p_{r}+1}^{n} f_{i, i}\right\rangle$. Then $\mathfrak{g}^{\mathfrak{r}} \cong \mathfrak{g l}_{p_{r}} \oplus \mathfrak{g}_{2 n-2 p_{r}}^{\phi}$. Since $L^{\mathfrak{r}}\left(\Lambda_{A}\right)$ is a finite dimensional $U\left(\mathfrak{g}^{\mathfrak{r}}, e\right)$-module by Corollary 3.14, we get that $L\left(A_{r-1}^{1-r}\right)$ is a finite dimensional $U\left(\mathfrak{g}^{\prime}, e^{\prime}\right)$-module, where $\mathfrak{g}^{\prime} \cong \mathfrak{g}_{2 n-2 p_{r}}^{\phi}$ and $e^{\prime} \in \mathfrak{g}^{\prime}$ is the nilpotent element of $\mathfrak{g}^{\prime}$ defined from the symmetric pyramid of $\mathbf{p}^{\prime}=\left(p_{1}^{2}, \ldots, p_{r-1}^{2}\right)$. So by induction we can apply some word $w$ in the elements of the set $\left\{c_{1}, \ldots, c_{d^{\prime}}\right\}$, where $d^{\prime}=d-1$ if $i_{d}=r$ and $d^{\prime}=d$ is $i_{d}<r$ to $A$ to yield an s-frame $B$ which is justified row equivalent to column strict. By replacing $A$ by $B$, and using Theorem 4.6, we can assume that $A_{r-1}^{1-r}$ is justified row equivalent to column strict.

Using Lemma 5.11 and Theorem 4.6, we see that if one adjusts $A$ so that the middle $2 r-2$ rows are left justified, row $-r$ is left justified with row $-r+1$, and row $r$ is right justified with row $r-1$, then the resulting diagram is row equivalent to column strict. Note that if 
$p_{r}=p_{r-1}$, then this implies that $A$ itself is justified row equivalent to column strict, so we assume that this is not the case.

Let $\mathbf{q}=\operatorname{part}(\operatorname{RS}(A))$. Now the above discussion and Lemma 4.4 show that

$$
\mathbf{q}^{T} \geq\left((2 r-1)^{2 p_{r}},(2 r-2)^{p_{r-1}-2 p_{r}},(2 r-4)^{p_{r-2}-p_{r-1}}, \ldots, 2^{p_{1}-p_{2}}\right)
$$

in the case that $p_{r} \leq p_{r-1} / 2$ and that

$$
\mathbf{q}^{T} \geq\left((2 r)^{2 p_{r}-p_{r-1}},(2 r-1)^{2\left(p_{r-1}-p_{r}\right)},(2 r-4)^{p_{r-2}-p_{r-1}}, \ldots, 2^{p_{1}-p_{2}}\right)
$$

in the case that $p_{r}>p_{r-1} / 2$. This implies that

$$
\mathbf{q} \leq\left(p_{1}^{2}, \ldots, p_{r-1}^{2}, 2 p_{r}\right)
$$

in the case that $p_{r} \leq p_{r-1} / 2$ and that

$$
\mathbf{q} \leq\left(p_{1}^{2}, \ldots, p_{r-1}^{2}, p_{r-1}, 2 p_{r}-p_{r-1}\right)
$$

in the case that $p_{r}>p_{r-1} / 2$. Since $A$ has increasing rows we also have that

$$
\mathbf{q} \geq\left(p_{1}^{2}, \ldots, p_{r}^{2}\right) .
$$

The content of $\mathbf{q}$ is defined at the end of $\$ 5.2$. From Corollaries 3.13 and 5.4 it follows that the length of the content of $\mathbf{q}$ is the same as the length of the content of $\mathbf{p}$.

Suppose for this paragraph that $\mathfrak{g}$ is of type C. The content of $\mathbf{p}$ has length $2 r+1$, which implies that

$$
\mathbf{q}=\left(p_{1}^{2}, \ldots, p_{r-1}^{2}, 2 p_{r}-a, a\right)
$$

for some $a$ where $1 \leq a \leq p_{r}$ in the case that $p_{r} \leq p_{r-1} / 2$ and that

$$
\mathbf{q}=\left(p_{1}^{2}, \ldots, p_{r-1}^{2}, p_{r-1}-a, 2 p_{r}-p_{r-1}+a\right)
$$

for some $a$ where $0 \leq a \leq p_{r-1}-p_{r}$ in the case that $p_{r}>p_{r-1} / 2$. The 3 first entries in the content of $\mathbf{p}$ are

$$
\left(0, \frac{p_{r}+1}{2}, \frac{p_{r}+1}{2}\right)
$$

when $p_{r}$ is odd and

$$
\left(0, \frac{p_{r}}{2}, \frac{p_{r}}{2}+1\right)
$$

when $p_{r}$ is even. This implies that

$$
a= \begin{cases}p_{r} & \text { if } p_{r} \leq p_{r-1} / 2, p_{r} \text { is odd, and } a \text { is odd; } \\ p_{r}-1 & \text { if } p_{r} \leq p_{r-1} / 2, p_{r} \text { is even, and } a \text { is odd; } \\ p_{r} & \text { if } p_{r} \leq p_{r-1} / 2, p_{r} \text { is even, and } a \text { is even; } \\ p_{r-1}-p_{r} & \text { if } p_{r}>p_{r-1} / 2, p_{r} \text { is odd, and } p_{r-1}-a \text { is odd; } \\ p_{r-1}-p_{r}-1 & \text { if } p_{r}>p_{r-1} / 2, p_{r} \text { is even, and } p_{r-1}-a \text { is odd; } \\ p_{r-1}-p_{r} & \text { if } p_{r}>p_{r-1} / 2, p_{r} \text { is even, and } p_{r-1}-a \text { is even. }\end{cases}
$$

It also implies that no such $a$ exists when $p_{r} \leq p_{r-1} / 2, p_{r}$ is odd, and $a$ is even; or when $p_{r}>p_{r-1} / 2, p_{r}$ is odd, and $p_{r-1}-a$ is even. Putting this all together shows that in all cases

$$
\mathbf{q}=\left(p_{1}^{2}, \ldots, p_{r-1}^{2}, p_{r}^{2}\right)
$$

or

$$
\mathbf{q}=\left(p_{1}^{2}, \ldots, p_{r-1}^{2}, p_{r}+1, p_{r}-1\right)
$$


Moreover, (5.15) is only possible if $p_{r}$ is even.

If $\mathfrak{g}$ is of type $\mathrm{D}$, then similar arguments show that $\mathbf{q}$ must be as in (5.14) or (5.15), and (5.15) can only occur if $p_{r}$ is odd.

If (5.14) holds, then $A$ is justified row equivalent to column strict by Theorem 4.6. So for the rest of the proof we assume that (5.15) holds. We also assumed above that $p_{r}<p_{r-1}$, which implies that $i_{d}=r$, so we can apply $c_{d}$ to $A$.

For the arguments below it is useful to note that

$$
\mathbf{q}^{T}=\left((2 r)^{p_{r}-1},(2 r-1)^{2},(2 r-2)^{p_{r-1}-p_{r}-1},(2 r-4)^{p_{r-2}-p_{r-1}}, \ldots, 2^{p_{1}-p_{2}}\right) .
$$

Let $A^{\prime}=\bar{s}_{1} \bar{s}_{2} \ldots \bar{s}_{r-1} A$, so that the shortest rows of $A^{\prime}$ are the middle two, and they have length $p_{r}$. Also let $F^{\prime}$ be the frame of $A^{\prime}$.

Let $\mathbf{r}=\operatorname{part}\left(\operatorname{RS}\left(A_{1}^{\prime-1}\right)\right)$. We aim to show that $\mathbf{r}=\left(p_{r}+1, p_{r}-1\right)$.

To do this first note that $\mathbf{r} \geq\left(p_{r}, p_{r}\right)$. If the first part of $\mathbf{r}$ were larger than $p_{r}+1$, then since the rows of $A^{\prime}$ are increasing, we would have

$$
\mathbf{q}>\left(p_{1}^{2}, \ldots, p_{r-1}^{2}, p_{r}+1, p_{r}-1\right),
$$

which contradicts (5.15).

Now suppose that $\mathbf{r}=\left(p_{r}^{2}\right)$. Using Lemma 5.11 and Theorem4.6 we see that $A^{\prime+}$ is justified row equivalent to column strict, which implies that we can find $p_{r}$ disjoint descending chains in word $\left(A^{\prime}\right)$ of length $2 r$. This implies that $\mathbf{q}^{T} \geq\left((2 r)^{p_{r}}, 1^{2 n-2 r p_{r}}\right)$ by Lemma 4.3, which contradicts (5.16).

Now the arguments in the previous two paragraphs prove that $\mathbf{r}=\left(p_{r}+1, p_{r}-1\right)$ as desired. Let $a_{1}<a_{2}<\cdots<a_{p_{r}}$ be the entries in row -1 of $A_{1}^{\prime-1}$. By Lemma 5.6 we have that the $\sharp$-element of $\left(a_{1}, \ldots, a_{p_{r}}\right)$ is defined, and we let $a_{j}$ be the $\sharp$-element. Thus $c \cdot A^{\prime}$ is defined, and by Lemma 5.6 we have that $\operatorname{part}\left(\operatorname{RS}\left(c \cdot A_{1}^{\prime-1}\right)\right)=\left(p_{r}, p_{r}\right)$.

Let $\mathbf{q}^{\sharp}=\operatorname{part}\left(\operatorname{RS}\left(c \cdot A^{\prime}\right)\right)$. Note that if $u, w$ are words of integers and $a, b \in \mathbb{Z}$ with $a<b$, then $\mathrm{RS}(u a b w) \geq \mathrm{RS}(u b a w)$ by Lemma 4.3, because every collection of disjoint increasing sequences in ubaw is a collection of disjoint increasing sequences in uabw. Now Remark 5.8 says that precisely 2 such swaps are required to get from something Knuth-equivalent to word $\left(A^{\prime}\right)$ to something Knuth-equivalent to word $\left(c \cdot A^{\prime}\right)$. Therefore, we have $\mathbf{q}^{\sharp} \leq \mathbf{q}$.

Now we have that

$$
\mathbf{q}^{\sharp}=\left(p_{1}^{2}, \ldots, p_{r-1}^{2}, p_{r}+1, p_{r}-1\right)
$$

or

$$
\mathbf{q}^{\sharp}=\left(p_{1}^{2}, \ldots, p_{r-1}^{2}, p_{r}, p_{r}\right) .
$$

¿From Remark 4.5 and (5.16), we see that we can find $p_{r}-1$ disjoint descending chains in the word $\left(A^{\prime}\right)$ of length $2 r$, and we can find 2 other decreasing chains of length $2 r-1$ which are disjoint from the chains of length $2 r$. These chains must include every number which occurs in $A_{1}^{\prime-1}$. Furthermore it is possible to adjust them so that one of the chains of length $2 r-1$ contains $a_{j}$, and other chain of length $2 r-1$ contains $-a_{j}$. To do this explicitly note that the existence of the chains of length $2 r$ and $2 r-1$ implies that we can form $p_{r}$ disjoint descending chains of length $r$ which end in $a_{1}, \ldots, a_{p_{r}}$, as well as $p_{r}$ disjoint descending chains of length $r$ which start in $-a_{p_{r}}, \ldots,-a_{1}$. Since the $\sharp$-element is defined, we can assign to each $i \in\left\{1, \ldots, p_{r}\right\} \backslash\{j\}$ some $i^{\prime} \in\left\{1, \ldots, p_{r}\right\} \backslash\{j\}$ such that $a_{i}>-a_{i^{\prime}}$. To be clear, this assignment can be made so that $i_{1}^{\prime}=i_{2}^{\prime}$ implies that $i_{1}=i_{2}$. Also note that in the type $\mathrm{D}$ case our zero convention from Algorithm 5.3 does not affect things here since in this case $p_{r}$ is odd, so by Lemma 5.5 we can make this assignment so that if $a_{i}=0$, then $a_{i^{\prime}} \neq 0$. Thus 
we can join $p_{r}-1$ of these chains together at $a_{i},-a_{i^{\prime}}$ for $i \in\left\{1, \ldots, p_{r}\right\} \backslash\{j\}$, to get $p_{r}-1$ disjoint descending chains of length $2 r$, and still have 2 other disjoint descending chains of length $r$, one of which ends in $a_{j}$, and one of which starts in $-a_{j}$.

Now after $c$ is applied to $A_{1}^{\prime-1}$ we have that $a_{j}$ now occurs in row 1 , while $-a_{j}$ now occurs in row -1 . All of the descending chains created above still exist, and now we can join the last 2 chains containing $-a_{j}$ and $a_{j}$ to form one more descending chain of length $2 r$. So $\left(\mathbf{q}^{\sharp}\right)^{T}$ is larger than or equal to the partition $\left((2 r)^{p_{r}}, 1^{2 n-2 r p_{r}}\right)$, which implies that $\mathbf{q}^{\sharp}=\left(p_{1}^{2}, \ldots, p_{r}^{2}\right)=\mathbf{p}$.

Using Lemma 4.8, we see that $\operatorname{RS}\left(c_{d} \cdot A\right)=\mathbf{p}$. Hence, by Theorem 4.6 it is justified row equivalent to column strict.

Theorem 5.13 and Lemma 4.13 immediately give the following corollary:

Corollary 5.17. Let $\mathfrak{g}, \mathbf{p}, A$, and $e$ be as in Theorem 5.13, and also suppose that all the parts of $\mathbf{p}$ have the same parity. Then the $U(\mathfrak{g}, e)$-module $L(A)$ is finite dimensional if and only if $A$ is $\tilde{C}$-conjugate to an s-table which is row equivalent to a column strict s-table.

Next we give a corollary of Theorem 5.13 saying that all finite dimensional irreducible $U(\mathfrak{g}, e)$-modules with integral central character can be obtained by restricting certain $U(\mathfrak{h})$ modules when all parts of $\mathfrak{p}$ have the same parity. In this case $\mathfrak{k}=0$, so $\tilde{\mathfrak{p}}=\mathfrak{p}$. The Miura map is by definition the composition of the inclusion $U(\mathfrak{g}, e) \hookrightarrow U(\mathfrak{p})$ with the surjection $U(\mathfrak{p}) \rightarrow U(\mathfrak{h})$. It is known that the Miura map is injective, see [Pr2, Remark 2.2], which allows us to restrict $U(\mathfrak{h})$-modules to $U(\mathfrak{g}, e)$.

Corollary 5.18. Let $\mathfrak{g}, \mathbf{p}$, and e be as in Theorem 5.13, and also suppose that all the parts of $\mathbf{p}$ have the same parity. Let $L$ be a finite dimensional irreducible $U(\mathfrak{g}, e)$-module with integral central character. Then there exists a finite dimensional $U(\mathfrak{h})$-module $M$ such that $L$ is a subquotient of the restriction of $M$ to $U(\mathfrak{g}, e)$.

Proof. First let $A \in \operatorname{Pyr}_{\phi}^{\mathrm{c}}(\mathbf{p})$, so by Lemma 4.13 there exists $B \in \bar{A}$ which is column strict as an s-table.

We claim that the weight $\lambda_{B} \in \mathfrak{t}^{*}$ is dominant for $\mathfrak{h}$ with respect to the Borel subalgebra $\mathfrak{b}^{h}$ of $\mathfrak{h}=\mathfrak{g}^{h}$. To see this, note that

$$
\tilde{\rho}+\gamma=\frac{1}{2}\left(\sum_{\substack{\alpha \in \Phi_{+} \\ \mathfrak{g}_{\alpha} \in \mathfrak{h}}} \alpha+\sum_{i=1}^{r} \beta_{i}\right) .
$$

Note that the first sum is the usual "choice of $\rho$ " for $\mathfrak{h}$, and the second sum is orthogonal to every root occurring in the first sum, so $\tilde{\rho}+\gamma$ is a "choice of $\rho$ " for $\mathfrak{h}$. So the highest weight $U(\mathfrak{h})$-module with highest weight $\lambda_{B}-(\tilde{\rho}+\gamma)$ with respect to the Borel subalgebra $\mathfrak{b}^{h}$ is finite dimensional. We denote this module by $M=L_{\mathfrak{h}}\left(\lambda_{B}\right)$ and let $v_{+} \in M$ be a highest weight vector. We can restrict $M$ to a $U(\mathfrak{g}, e)$-module through the Miura map. It is clear that $v_{+} \in M$ lies in a maximal $\mathfrak{t}^{e}$-weight space $M_{\mu}$, where $\mu \in\left(\mathfrak{t}^{e}\right)^{*}$. Thus $M_{\mu}$ can be viewed as a $U\left(\mathfrak{g}_{0}, e\right)$-module as in $\$ 3.3$. As such it is clear that $v_{+}$spans a one dimensional $U\left(\mathfrak{g}_{0}, e\right)$ submodule. Through the isomorphism $\xi_{\tilde{\rho}}: U\left(\mathfrak{g}_{0}, e\right) \rightarrow S(\mathfrak{t})^{W_{0}}$ from (3.11), this identifies with the $S(\mathfrak{t})^{W_{0}}$-module $V_{\Lambda_{A}}$. It follows that the $U(\mathfrak{g}, e)$-submodule of $M$ generated by $v_{+}$is a highest weight $U(\mathfrak{g}, e)$-module of type $\Lambda_{A}$, and thus has a quotient isomorphic to $L(A)$. 
Now let $A \in \operatorname{Pyr}_{\phi}^{\leq}(\mathbf{p})$ such that $L(A)$ is finite dimensional. Then by Theorem 5.13, there exists $c \in \tilde{C}$ such that $c \cdot A$ is row equivalent to column strict. Therefore, $L(c \cdot A)$ is isomorphic to a subquotient of a finite dimensional $U(\mathfrak{h})$-module $M$ by the previous paragraph. We write $c \cdot M$ for the $U(\mathfrak{h})$-module obtained by twisting $M$ by $c$, which is defined similarly to the case of $U(\mathfrak{g}, e)$-modules in 93.6 . Now using the result in BroG that $L(A) \cong c \cdot L(c \cdot A)$, we see that $L(A)$ is isomorphic to a subquotient of $c \cdot M$. (We note that $c \cdot M$ is isomorphic to $M$ if $c \in C$.)

Remark 5.19. The hypothesis that $A$ in Theorem 5.13 has entries all from $\mathbb{Z}$, or all from $\mathbb{Z}+\frac{1}{2}$, ensures that $\lambda_{A} \in \mathfrak{t}_{\mathbb{Z}}^{*}$. In the example below we demonstrate that this hypothesis is necessary. As usual for complex numbers $x, y$ we say $x<y$ if $y-x \in \mathbb{Z}_{>0}$. If we allow tables to have entries to have entries from $\mathbb{C}$, then we say that a (justified) table is column strict provided its columns are decreasing with respect to this partial order. Now if $\mathfrak{g}=\mathfrak{s p}$ and $e \in \mathfrak{g}$ has Jordan type $\left(2^{2}\right)$, then by [Bro2, Theorem 1.2] the $U(\mathfrak{g}, e)$-module $L(A)$, where

$$
A=\begin{array}{|c|c|}
\hline \pi & 1 \\
\hline-1 & -\pi \\
\hline
\end{array}
$$

is finite dimensional, however $A$ is not $\tilde{C}$-conjugate to a row equivalent to column strict s-table.

The following corollary is immediate from Theorem 5.13 and the map.$^{\dagger}$ from (2.4). Recall that in the type D case $C \subset \tilde{C}$ is a subgroup of index 2. With this in mind, we use $\operatorname{Pyr}_{+, s}^{\mathrm{c}}(\mathbf{p})$ to denote the elements $A$ of $\mathrm{Pyr}_{+}^{\mathrm{c}}(\mathbf{p})$ for which there exists $c \in \tilde{C} \backslash C$ such that $c \cdot A=A$. We also note that if $A$ has no boxes filled with 0 then $A \notin \operatorname{Pyr}_{+, s}^{\mathrm{c}}(\mathbf{p})$.

Corollary 5.20. Let $\mathfrak{g}=\mathfrak{s p}_{2 n}$ or $\mathfrak{s o}_{2 n}$, and let e be an even multiplicity nilpotent element of $\mathfrak{g}$. If $\mathfrak{g}=\mathfrak{s p}_{2 n}$, then

$$
\left\{\operatorname{Ann}_{U(\mathfrak{g})} L\left(\lambda_{A}\right) \mid A \in \mathrm{Pyr}_{-}^{\mathrm{c}}(\mathbf{p})\right\}
$$

is a complete set of pairwise distinct primitive ideals of $U(\mathfrak{g})$ with integral central character and associated variety $\overline{G \cdot e}$.

If $\mathfrak{g}=\mathfrak{s o}_{2 n}$, then

$$
\begin{aligned}
\left\{\operatorname{Ann}_{U(\mathfrak{g})}\right. & \left.L\left(\lambda_{A}\right) \mid A \in \mathrm{Pyr}_{+, s}^{\mathrm{c}}(\mathbf{p})\right\} \\
& \cup\left\{\operatorname{Ann}_{U(\mathfrak{g})} L\left(\lambda_{A}\right) \mid A \in \mathrm{Pyr}_{+}^{\mathrm{c}}(\mathbf{p}) \backslash \mathrm{Pyr}_{+, s}^{\mathrm{c}}(\mathbf{p})\right\} \\
& \cup\left\{\operatorname{Ann}_{U(\mathfrak{g})} L\left(\lambda_{c_{1} \cdot A} \mid A \in \mathrm{Pyr}_{+}^{\mathrm{c}}(\mathbf{p}) \backslash \mathrm{Pyr}_{+, s}^{\mathrm{c}}(\mathbf{p})\right\}\right.
\end{aligned}
$$

is a complete set of pairwise distinct primitive ideals of $U(\mathfrak{g})$ with integral central character and associated variety $\overline{G \cdot e}$.

\section{REFERENCES}

[BB] J. Brown and J. Brundan, Elementary invariants for centralizers of nilpotent matrices, J. Austral. Math. Soc. 86 (2009), 1-15, math.RA/0611024.

[Bro1] J. Brown, Twisted Yangians and finite W-algebras, Transform. Groups 14 (2009), no. 1, 87-114.

[Bro2] - Representation theory of rectangular finite $W$-algebras, preprint, arXiv:1003.2179v1 (2010).

[BroG] J. Brown and S. M. Goodwin, Changing the highest weight theory for finite $W$-algebras, in preparation (2010). 
[Bru1] J. Brundan, Centers of degenerate cyclotomic Hecke algebras and parabolic category $\mathcal{O}$, Represent. Theory 12 (2008), 236-259.

[Bru2] $\quad$ Symmetric functions, parabolic category $\mathcal{O}$, and the Springer fiber, Duke Math. J. 143 (2008), 41-79.

[BruG] J. Brundan and S. M. Goodwin, Good grading polytopes, Proc. London Math. Soc. 94 (2007), $155-180$.

[BGK] J. Brundan, S. M. Goodwin and A. Kleshchev, Highest weight theory for finite $W$-algebras, Internat. Math. Res. Notices, 15 (2008), Art. ID rnn051.

[BK1] J. Brundan and A. Kleshchev, Shifted Yangians and finite W-algebras, Adv. Math. 200 (2006), 136-195.

[BK2] - Representations of shifted Yangians and finite $W$-algebras, Mem. Amer. Math. Soc. 196 (2008).

[BK3] _ Schur-Weyl duality for higher levels, Selecta Math 14 (2008), 1-57 (2008).

[BV] D. Barbasch and D. Vogan, Primitive ideals and orbital integrals in complex classical groups, Math. Ann. 259 (1982), 153-199.

$\left[\mathrm{D}^{3} \mathrm{HK}\right]$ A. D'Andrea, C. De Concini, A. De Sole, R. Heluani and V. Kac, Three equivalent definitions of finite $W$-algebras, appendix to [DK].

[DK] A. De Sole and V. Kac, Finite vs affine W-algebras, Jpn. J. Math. 1 (2006), 137-261.

[Du] M. Duflo, Sur la classification des idéaux primitif dans l'algebre enveloppante d'une algebre de Lie semisimple, Ann. of Math. 105 (1977), 107-120.

[EK] A. Elashvili and V. Kac, Classification of good gradings of simple Lie algebras, in Lie groups and invariant theory (E. B. Vinberg ed.), pp. 85-104, Amer. Math. Soc. Transl. 13, AMS, 2005.

[F] W. Fulton, Young tableaux, London Math. Soc. Stud. Texts 35, Cambridge University Press, Cambridge, UK, 1997..

[GG] W. L. Gan and V. Ginzburg, Quantization of Slodowy slices, Internat. Math. Res. Notices 5 (2002), 243-255.

[Gi] V. Ginzburg, Harish-Chandra bimodules for quantized Slodowy slices, Represent. Theory 13 (2009), 236-271.

[Gr] C. Green, Some partitions associated with a partially ordered set, J. of Combin. Theory, Ser. A 20 (1976), 69-79.

[Ja] J. C. Jantzen, Nilpotent orbits in representation theory, Progress in Math., vol. 228, Birkhäuser, 2004.

[Jo1] A. Joseph, Towards the Jantzen conjecture III, Compositio Math. 41 (1981), 23-30.

[Jo2] _ On the associated variety of a primitive ideal, J. Algebra 93 (1985), 509-523.

[Ko] B. Kostant, On Whittaker modules and representation theory, Invent. Math. 48 (1978), 101-184.

[Lo1] I. Losev, Quantized symplectic actions and W-algebras, J. Amer. Math. Soc. 23 (2010), no. 1, 35-59.

[Lo2] - Finite dimensional representations of $W$-algebras, preprint, arXiv:0807.1023 (2008).

[Lo3] - On the structure of the category $\mathcal{O}$ for $W$-algebras, preprint, arXiv:0812.1584 (2008).

[Lo4] Finite $W$-algebras, preprint, arXiv:1003.5811 (2010).

[Lu] G. Lusztig, A class of irreducible representations of a Weyl group, Proc. Nedeerl. Akad., Series A 82 (1979), 323-335.

[MS] D. Miličíc and W. Soergel, The composition series of modules induced from Whittaker modules, Comment. Math. Helv. 72 (1997), 503-520.

[Pr1] A. Premet, Special transverse slices and their enveloping algebras, Adv. in Math. 170 (2002), 1-55.

[Pr2] Enveloping algebras of Slodowy slices and the Joseph ideal, J. Eur. Math. Soc. 9 (2007), 487-543.

[Pr3] $\quad$ Primitive ideals, non-restricted representations and finite $W$-algebras, Mosc. Math. J. 7 (2007), 743-762.

[Pr4] Commutative quotients of finite $W$-algebras, preprint, arXiv:0809.0663 (2008).

[Sk] S. Skryabin, A category equivalence, appendix to [Pr1].

School of Mathematics, University of Birmingham, Birmingham, B15 3LX, UK

E-mail address: brownjs@for.mat.bham.ac.uk, goodwin@for.mat.bham.ac.uk 